University of Tennessee Health Science Center

UTHSC Digital Commons

\title{
Pharmacokinetic and Pharmacodynamic Studies of a Novel Spectinamide Series of Antituberculosis Agents
}

\author{
V. N. R. Pavan Kumar Vaddady \\ University of Tennessee Health Science Center
}

Follow this and additional works at: https://dc.uthsc.edu/dissertations

Part of the Bacterial Infections and Mycoses Commons, Medicinal and Pharmaceutical Chemistry Commons, and the Pharmaceutics and Drug Design Commons

\section{Recommended Citation}

Vaddady, V. N. R. Pavan Kumar, "Pharmacokinetic and Pharmacodynamic Studies of a Novel Spectinamide Series of Antituberculosis Agents" (2011). Theses and Dissertations (ETD). Paper 278. http://dx.doi.org/10.21007/etd.cghs.2011.0331.

This Dissertation is brought to you for free and open access by the College of Graduate Health Sciences at UTHSC Digital Commons. It has been accepted for inclusion in Theses and Dissertations (ETD) by an authorized administrator of UTHSC Digital Commons. For more information, please contact jwelch30@uthsc.edu. 


\title{
Pharmacokinetic and Pharmacodynamic Studies of a Novel Spectinamide Series of Antituberculosis Agents
}

\begin{abstract}
Spectinamides are novel amide derivatives of the antibiotic spectinomycin that have emerged as a new class of agents to treat tuberculosis. These agents showed potent in vitro activity against Mycobacterium tuberculosis (MTB) compared to spectinomycin and in a preliminary in vivo study in interferon gamma (IFN-Y) knockout mice, spectinamide Lee1329 reduced the lung bacillary load of TB comparable to streptomycin. We hypothesized that the application of an iterative pharmacokinetics and pharmacodynamics (PK/PD) guided approach would facilitate the optimization of these lead compounds suitable for further development.

A series of in vitro experiments including parallel artificial membrane permeability assay (PAMPA), microsomal metabolic stability using rat liver microsomes and protein binding assay were designed to characterize the in vivo biopharmaceutic and pharmacokinetic behavior. Drug uptake studies into Mycobacterium bovis BCG and into $\mathrm{J} 774$ murine macrophages were performed to understand reasons for improved activity of spectinamides and to evaluate their potential to target primary TB infection that resides in macrophages. In vivo pharmacokinetic studies were carried out in rats by intravenous (10 mg/ $\mathrm{Kg}$ ) and oral administration ( $100 \mathrm{mg} / \mathrm{Kg}$ ) of the compounds. LC-MS/MS assays were developed to quantify concentrations in test samples obtained from these studies.
\end{abstract}

Spectinamides exhibit low to intermediate plasma protein binding and were found to be metabolically stable. Following intravenous administration, spectinamides were relatively widely distributed ( 0.36 to $1.15 \mathrm{~L} / \mathrm{Kg}$ ) with short half-lives $(0.43$ to $0.62 \mathrm{hr}$ ). Mean systemic clearance ranged between 0.36 and 0.89 $\mathrm{L} / \mathrm{hr} / \mathrm{Kg}$ with a significant fraction of drug eliminated unchanged in urine (0.46 to 1.0). Spectinamides exhibited a renal excretion ratio greater than one indicating filtration and active secretion as the net renal elimination process. In the uptake experiments, the spectinamides exhibited 3-4 times higher uptake into murine macrophages compared to streptomycin and showed nearly four times higher uptake into $M$. bovis BCG compared to spectinomycin, which may in part explain their increased activity compared to spectinomycin.

In a previously reported in vitro PK/PD system, PK concentration-time profiles were simulated on the basis of the in vivo clearance of rats obtained from the PK studies and different daily doses of $0.4,2,10$ and $50 \mathrm{mg} / \mathrm{Kg} /$ day of Lee 1445 were added as QD, BID or TID regimens. The time-kill effect of these regimens was studied on the growing $M$. bovis BCG present in the system. A semi-mechanistic model incorporating a logistic function for growth of mycobacteria in the absence of drug and an inhibitory sigmoidal $\mathrm{E}_{\max }$ model with a delay function for the time-kill effect of the drug was fit to the data and the in vitro PK/PD parameters were determined. Since the in vivo efficacy model of tuberculosis infection was the gamma knock-out (GKO) mice, a PK bridging study was performed in mice following intraperitoneal administration of $20 \mathrm{mg} / \mathrm{Kg}$ Lee 1445 . Simulations for various dosing regimens were performed using the obtained mouse PK parameters and the in vitro PK/PD parameters that were incorporated into a combined PK/PD model to predict the efficacy in terms of reduction of bacterial counts measured in log $\mathrm{CFU} / \mathrm{mL}$. Based on the results of the simulations, an optimal dose was chosen for the in vivo efficacy study.

The results from the in vitro PK/PD studies showed that QD was marginally effective. The same total daily dose administered BID showed a marked reduction in mycobacterial counts. TID dosing did not show a significant difference in time-kill compared to BID regimen. The maximum growth rate constant $\left(\mathrm{K}_{0}\right)$ was estimated as $0.0274 \mathrm{hr}^{-1}$ which corresponds to a maximum doubling time of $25.3 \mathrm{hr}$ that is consistent 
with the commonly observed in vitro doubling times of 20-24 hr. A maximum bacterial kill rate ( $\left.I_{\max }\right)$ was calculated as $0.0566 \mathrm{hr}^{-1}$. The free drug concentration required to produce half-maximum inhibition (IC50) was calculated as $2.62 \mathrm{mg} / \mathrm{L}$. The delay rate constant for the initial kill was found to be $0.0245 \mathrm{hr}^{-1}$. The PK bridging study showed that the Lee1445 half-life was shorter in mice $(0.25 \mathrm{hr})$ compared to rats $(0.43$ $\mathrm{hr}$ ). Simulations based on the parameters obtained from the mouse PK bridging study and the in vitro PK/ PD model suggested that a total daily dose of $200 \mathrm{mg} / \mathrm{Kg} /$ day and $400 \mathrm{mg} / \mathrm{Kg} /$ day administered BID would be optimal and result in approximately 2 and 3 log reduction in bacterial counts respectively after seven days of therapy and hence were chosen for in vivo efficacy studies in mice.

In summary, we have successfully developed a series of biopharmaceutic, PK and PD experiments that help in an iterative PK/PD guided approach for development of spectinamides, a novel class of antituberculosis agents. The in vivo PK and in vitro PK/PD parameters obtained from these studies provide a basis for optimal compound as well as dose selection.

\section{Document Type}

Dissertation

Degree Name

Doctor of Philosophy (PhD)

\section{Program}

Pharmaceutical Sciences

\section{Research Advisor}

Bernd Meibohm, Ph.D.

\section{Keywords}

In Vitro PK/PD Model Pharmacodynamics, Pharmacokinetics, Pharmacometrics, Tuberculosis

\section{Subject Categories}

Bacterial Infections and Mycoses | Diseases | Medicinal and Pharmaceutical Chemistry | Medicine and Health Sciences | Pharmaceutics and Drug Design | Pharmacy and Pharmaceutical Sciences 
PHARMACOKINETIC AND PHARMACODYNAMIC STUDIES OF A NOVEL SPECTINAMIDE SERIES OF ANTITUBERCULOSIS AGENTS

\author{
A Dissertation \\ Presented for \\ The Graduate Studies Council \\ The University of Tennessee \\ Health Science Center \\ In Partial Fulfillment \\ Of the Requirements for the Degree \\ Doctor of Philosophy \\ From The University of Tennessee
}

By

V. N. R. Pavan Kumar Vaddady

May 2011 
Portions of Chapter 1 (C) 2010 by Future Science Ltd. All other material (c) 2011 by V. N. R. Pavan Kumar Vaddady. All rights reserved. 
To my parents, sister and fiancée for their endless love and support

$$
\text { and }
$$

To all my teachers who taught from their hearts and not merely from the books 


\section{ACKNOWLEDGEMENTS}

The daunting task of obtaining my Doctorate in Philosophy could not have come true without the tremendous support and encouragement from so many people. First and foremost, I would like to express my deepest gratitude and sincere appreciation to my mentor Dr. Bernd Meibohm for providing me with an opportunity to work in his lab and being my biggest advocate throughout this endeavor. I am thankful to him for instilling in me the qualities of being a good scientist. His co-operation and continued support have been major driving forces throughout my graduate career at the University of Tennessee. I would also like to thank my committee members, Dr. Richard Lee, Dr. Charles Ryan Yates, Dr. Phillip D. Rogers, Dr. James E. Bina and Dr. Terreia Jones for their invaluable suggestions, guidance and assistance over the years.

I would like to especially acknowledge Dr. Richard Lee for providing me with the compounds and allowing me to use the resources in his laboratory without which my dissertation work would not have been possible. I am indebted to Dr. Julian Hurdle, Dr. Jiuyu Liu, Dr. Rakesh, Dr. Sourav Das, Robin Lee, Amy Heathcott, and Dr. Lei Yang at the Department of Chemical Biology and Therapeutics, St. Jude Children's Research Hospital and to my lab mates Dr. Nageshwar Budha, Dr. Dorababu Madhura, Josiah Ryman and Ashit Trivedi for all their support and help in my research work. I am also thankful to our collaborators Dr. Anne Lenaerts at the Department of Microbiology, Immunology and Pathology, Colorado State University for conducting the in vivo efficacy studies in TB infected mice, Dr. Erik Boettger at the Institute of Medical Microbiology, University of Zurich, Switzerland and Dr. Jose-Antonio Enriquez, Universidad de Zaragoza, Spain for their input on the microbiological aspects of these agents.

I would like to thank the National Institutes of Health and the American Lebanese Syrian Associated Charities (ALSAC) for providing the financial support to my research work. My special thanks to Dr. Jack Cook, Dr. Sriram Krishnaswami and Dr. Pankaj Gupta for providing me an opportunity to work as an intern at Clinical Pharmacology, Pfizer Inc., New London, CT.

A special thanks to my friends and colleagues, Nitin, Shailly, Nate, Maggie, Yi, Wararat, Satyendra, Karin, Chaela, Fei, Les, Faith, Lisa, Paras, Vinay, Puvi and Praveen for their support and wonderful company over all these years. I also extend my gratitude to the rest of my friends for all their friendship and for making my stay in Memphis a memorable experience.

I would like to thank my parents, sister and my fiancée; no words would be enough to thank their support and patience during the completion of this dissertation. I would also like to thank all my teachers from the kindergarten to the graduate school for igniting in me the light of knowledge. Finally, I would like to thank Almighty for everything that I have today. 


\begin{abstract}
Spectinamides are novel amide derivatives of the antibiotic spectinomycin that have emerged as a new class of agents to treat tuberculosis. These agents showed potent in vitro activity against Mycobacterium tuberculosis (MTB) compared to spectinomycin and in a preliminary in vivo study in interferon gamma (IFN- $\gamma$ ) knockout mice, spectinamide Lee1329 reduced the lung bacillary load of TB comparable to streptomycin. We hypothesized that the application of an iterative pharmacokinetics and pharmacodynamics (PK/PD) guided approach would facilitate the optimization of these lead compounds suitable for further development.
\end{abstract}

A series of in vitro experiments including parallel artificial membrane permeability assay (PAMPA), microsomal metabolic stability using rat liver microsomes and protein binding assay were designed to characterize the in vivo biopharmaceutic and pharmacokinetic behavior. Drug uptake studies into Mycobacterium bovis BCG and into J774 murine macrophages were performed to understand reasons for improved activity of spectinamides and to evaluate their potential to target primary TB infection that resides in macrophages. In vivo pharmacokinetic studies were carried out in rats by intravenous (10 $\mathrm{mg} / \mathrm{Kg}$ ) and oral administration $(100 \mathrm{mg} / \mathrm{Kg}$ ) of the compounds. LC-MS/MS assays were developed to quantify concentrations in test samples obtained from these studies.

Spectinamides exhibit low to intermediate plasma protein binding and were found to be metabolically stable. Following intravenous administration, spectinamides were relatively widely distributed ( 0.36 to $1.15 \mathrm{~L} / \mathrm{Kg}$ ) with short half-lives $(0.43$ to $0.62 \mathrm{hr})$. Mean systemic clearance ranged between 0.36 and $0.89 \mathrm{~L} / \mathrm{hr} / \mathrm{Kg}$ with a significant fraction of drug eliminated unchanged in urine (0.46 to 1.0). Spectinamides exhibited a renal excretion ratio greater than one indicating filtration and active secretion as the net renal elimination process. In the uptake experiments, the spectinamides exhibited 3-4 times higher uptake into murine macrophages compared to streptomycin and showed nearly four times higher uptake into M. bovis BCG compared to spectinomycin, which may in part explain their increased activity compared to spectinomycin.

In a previously reported in vitro $\mathrm{PK} / \mathrm{PD}$ system, $\mathrm{PK}$ concentration-time profiles were simulated on the basis of the in vivo clearance of rats obtained from the PK studies and different daily doses of $0.4,2,10$ and $50 \mathrm{mg} / \mathrm{Kg} /$ day of Lee 1445 were added as QD, BID or TID regimens. The time-kill effect of these regimens was studied on the growing M. bovis BCG present in the system. A semi-mechanistic model incorporating a logistic function for growth of mycobacteria in the absence of drug and an inhibitory sigmoidal $\mathrm{E}_{\max }$ model with a delay function for the time-kill effect of the drug was fit to the data and the in vitro PK/PD parameters were determined. Since the in vivo efficacy model of tuberculosis infection was the gamma knock-out (GKO) mice, a PK bridging study was performed in mice following intraperitoneal administration of $20 \mathrm{mg} / \mathrm{Kg}$ Lee 1445 . Simulations for various dosing regimens were performed using the obtained mouse PK parameters and the in vitro PK/PD parameters that were incorporated into a combined $\mathrm{PK} / \mathrm{PD}$ model to predict the efficacy in terms of reduction of bacterial counts measured in 
$\log \mathrm{CFU} / \mathrm{mL}$. Based on the results of the simulations, an optimal dose was chosen for the in vivo efficacy study.

The results from the in vitro PK/PD studies showed that QD was marginally effective. The same total daily dose administered BID showed a marked reduction in mycobacterial counts. TID dosing did not show a significant difference in time-kill compared to BID regimen. The maximum growth rate constant $\left(\mathrm{K}_{0}\right)$ was estimated as $0.0274 \mathrm{hr}^{-1}$ which corresponds to a maximum doubling time of $25.3 \mathrm{hr}$ that is consistent with the commonly observed in vitro doubling times of 20-24 hr. A maximum bacterial kill rate $\left(I_{\max }\right)$ was calculated as $0.0566 \mathrm{hr}^{-1}$. The free drug concentration required to produce half-maximum inhibition $\left(\mathrm{IC}_{50}\right)$ was calculated as $2.62 \mathrm{mg} / \mathrm{L}$. The delay rate constant for the initial kill was found to be $0.0245 \mathrm{hr}^{-1}$. The PK bridging study showed that the Lee 1445 half-life was shorter in mice $(0.25 \mathrm{hr})$ compared to rats $(0.43 \mathrm{hr})$. Simulations based on the parameters obtained from the mouse PK bridging study and the in vitro $\mathrm{PK} / \mathrm{PD}$ model suggested that a total daily dose of $200 \mathrm{mg} / \mathrm{Kg} /$ day and 400 $\mathrm{mg} / \mathrm{Kg} /$ day administered BID would be optimal and result in approximately 2 and $3 \log$ reduction in bacterial counts respectively after seven days of therapy and hence were chosen for in vivo efficacy studies in mice.

In summary, we have successfully developed a series of biopharmaceutic, PK and PD experiments that help in an iterative PK/PD guided approach for development of spectinamides, a novel class of antituberculosis agents. The in vivo $\mathrm{PK}$ and in vitro $\mathrm{PK} / \mathrm{PD}$ parameters obtained from these studies provide a basis for optimal compound as well as dose selection. 


\section{TABLE OF CONTENTS}

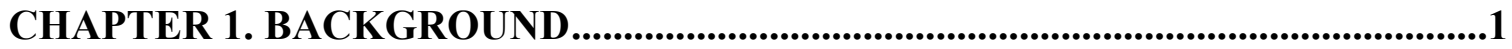

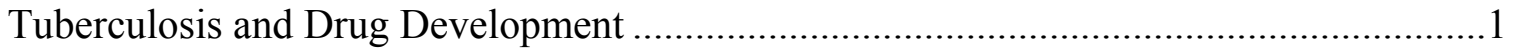

Pharmacokinetic/Pharmacodynamic Parameters of Antituberculosis Agents .....................2

Pharmacokinetics ............................................................................................

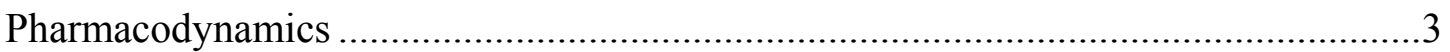

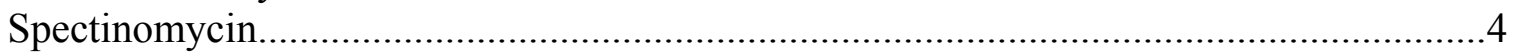

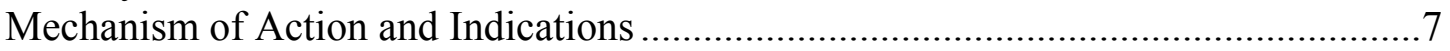

Preclinical and Veterinary Pharmacokinetics ..........................................................

Human Pharmacokinetics …………………………...................................

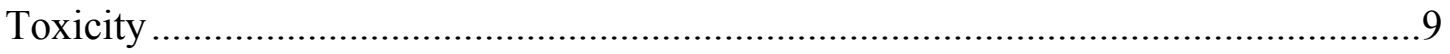

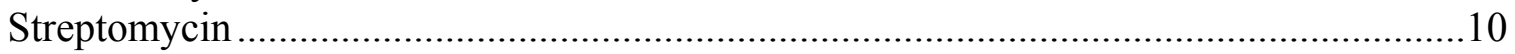

Mechanism of Action and Indications ..................................................................10

Preclinical and Veterinary Pharmacokinetics ............................................................10

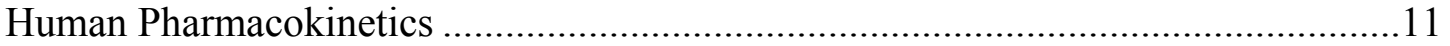

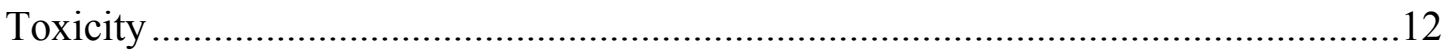

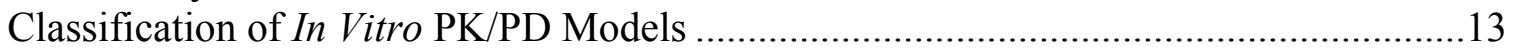

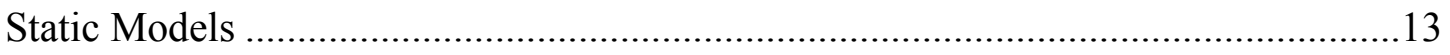

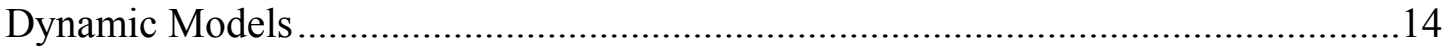

Perspectives Relevant to Drug Development …………............................................17

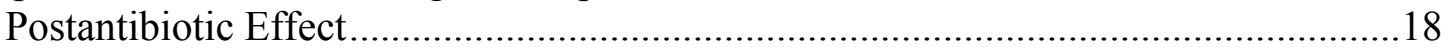

Evaluation of Combination Therapy...................................................................19

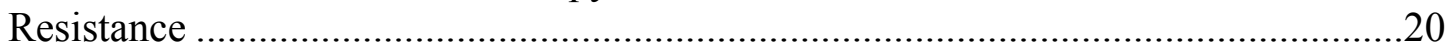

Pharmacokinetic/Pharmacodynamic Data Analysis ........................................................21

Mechanism Based Modeling for Estimating PK/PD Parameters ....................................22

Empirical Modeling for Identifying Appropriate PK/PD Indices....................................25

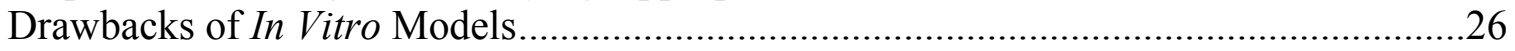

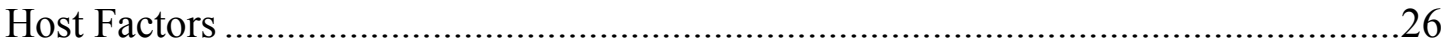

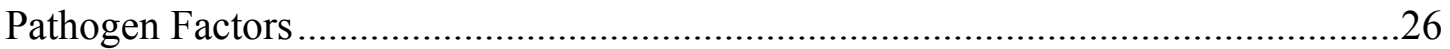

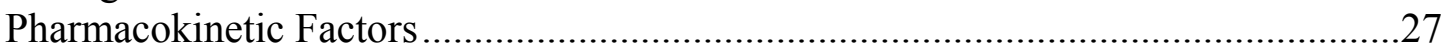

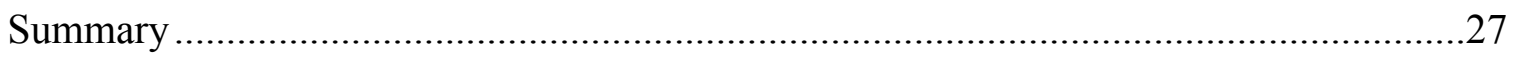

CHAPTER 2. RESEARCH HYPOTHESIS....................................................................29

CHAPTER 3. PHARMACOKINETIC EVALUATION OF

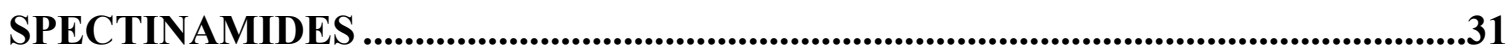

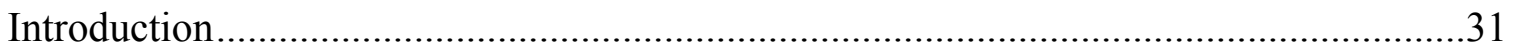

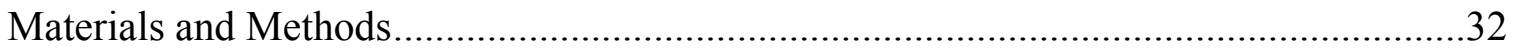

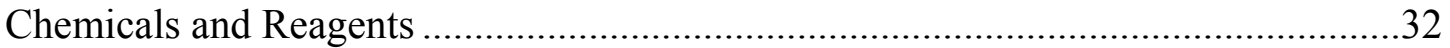

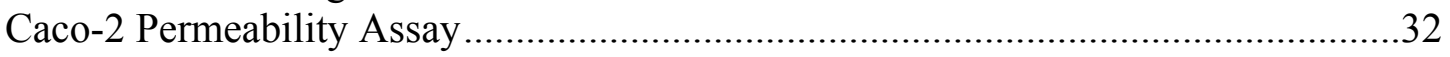

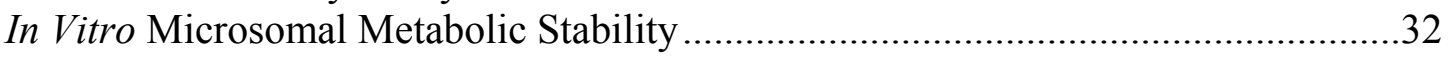

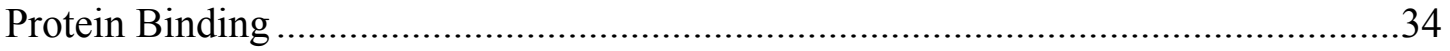

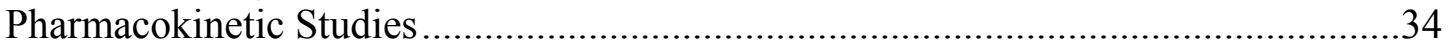

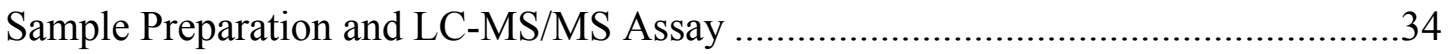




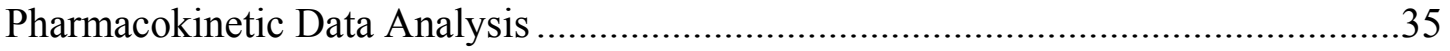

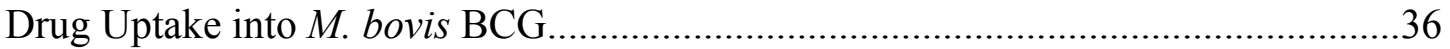

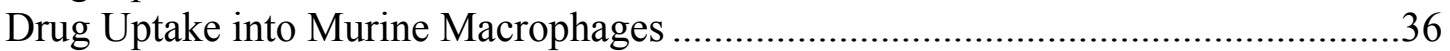

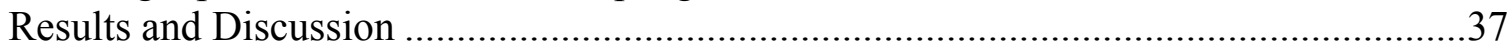

LC-MS/MS Assay Performance ............................................................................

Biopharmaceutical Properties ..................................................................................

In Vitro Microsomal Metabolic Stability ..................................................................

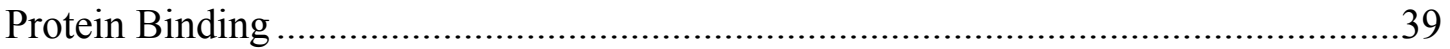

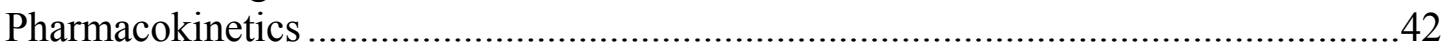

Drug Uptake into M. bovis BCG............................................................................49

Drug Uptake into Murine Macrophages .................................................................53

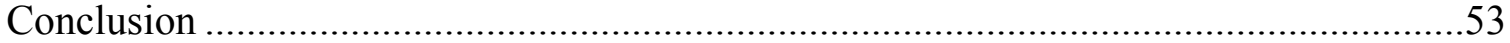

\section{CHAPTER 4. PHARMACODYNAMIC EVALUATION AND DOSE} OPTIMIZATION OF LEE1445 USING AN IN VITRO PK/PD MODEL ..................57

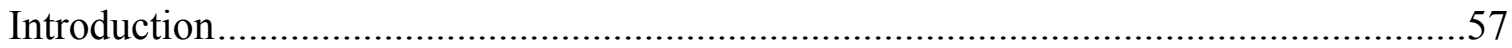

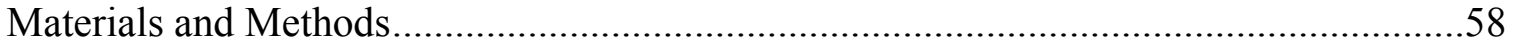

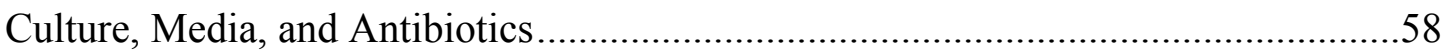

Minimum Inhibitory Concentration of Lee1445 ......................................................58

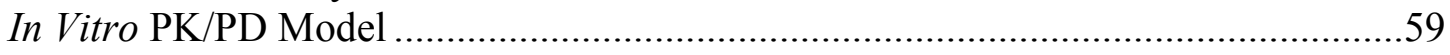

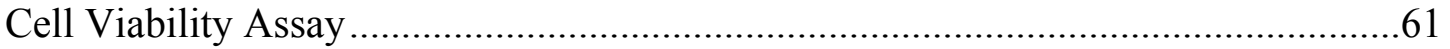

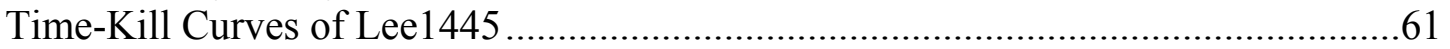

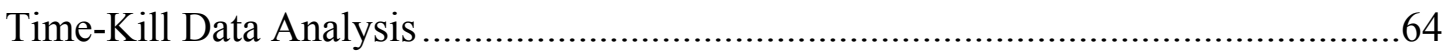

In Vivo Pharmacokinetic Bridging Study in Mice ..................................................65

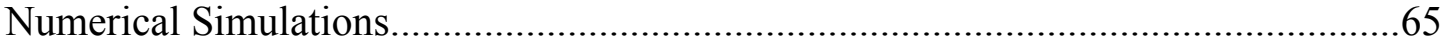

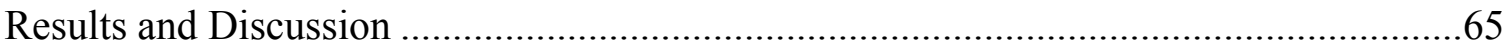

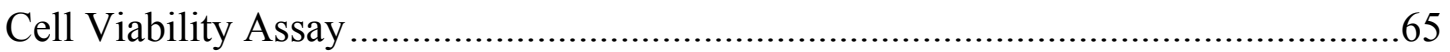

Time-Kill Curves and PK/PD Parameters ...............................................................67

In Vivo Pharmacokinetic Bridging Study in Mice ...................................................69

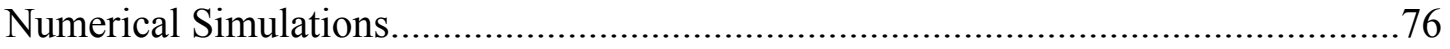

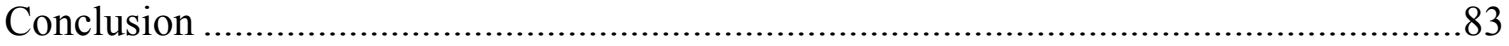

CHAPTER 5. SUMMARY OF DISSERTATION RESEARCH..................................84

LIST OF REFERENCES..................................................................................................

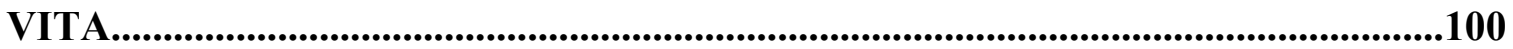




\section{LIST OF TABLES}

Table 1-1. $\quad$ Reported PK/PD parameters of antituberculosis drugs ........................... 6

Table 3-1. Physicochemical and biopharmaceutical properties of spectinamides ..... 38

Table 3-2. Metabolic half-life of spectinamides in presence of human and mouse liver microsomes .............................................................................. 41

Table 3-3. Protein binding of spectinamides in human, rat and mouse plasma ........ 43

Table 3-4. Pharmacokinetic parameters of spectinamides following intravenous

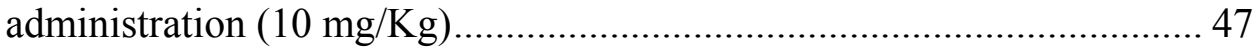

Table 3-5. Pharmacokinetic parameters of spectinamides following oral

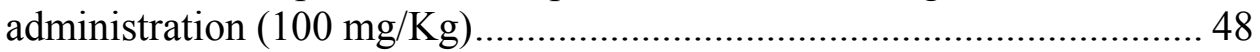

Table 3-6. $\quad$ Drug uptake into M. bovis BCG ..................................................... 52

Table 3-7. Drug uptake into the J774 murine macrophage cell line ........................ 55

Table 4-1. Dosing regimens tested in the in vitro PK/PD model ............................. 62

Table 4-2. In vitro PK/PD parameter estimates of Lee1445 ................................... 70

Table 4-3. Parameters for empirical PK/PD index T>MIC based on day 6 data....... 73

Table 4-4. Lee1445 in vivo pharmacokinetic differences between rat and mouse..... 75 


\section{LIST OF FIGURES}

Figure 1-1. Commonly used pharmacokinetic/pharmacodynamic indices.................... 5

Figure 1-2. In vitro PK/PD models for antituberculosis agents................................... 15

Figure 3-1. Chemical structures of spectinomycin, first generation 2-heteroaryl acetic acid amide analogs (Lee1329, Lee1443, Lee1445 and Lee1544), second generation fused ring analog (Lee1465) and second generation phenyl amino thiazole analog (Lee1540)................................................. 33

Figure 3-2. In vitro rat liver microsomal metabolic stability of spectinamides........... 40

Figure 3-3. Measured plasma concentration-time profiles (mean $\pm \mathrm{SD}$ ) after intravenous (10 mg/Kg, Panel A) or oral (100 mg/Kg, Panel B) administration of Lee1329 in rats

Figure 3-4. Measured plasma concentration-time profiles (mean $\pm \mathrm{SD}$ ) after 10 $\mathrm{mg} / \mathrm{Kg}$ intravenous administration of Lee1443 (Panel A) and Lee1445

(Panel B) in rats 45

Figure 3-5. Measured plasma concentration-time profiles (mean $\pm \mathrm{SD}$ ) after intravenous $(10 \mathrm{mg} / \mathrm{Kg}$, Panel A) or oral $(100 \mathrm{mg} / \mathrm{Kg}$, Panel B) administration of Lee1544 in rats 46

Figure 3-6. Measured plasma concentration-time profiles (mean $\pm \mathrm{SD}$ ) after intravenous (10 mg/Kg, Panel A) or oral (100 mg/Kg, Panel B) administration of Lee1465 in rats

Figure 3-7. Measured plasma concentration-time profiles (mean $\pm \mathrm{SD}$ ) after intravenous (10 mg/Kg, Panel A) or oral (100 mg/Kg, Panel B) administration of Lee1540 in rats 51

Figure 3-8. Cytotoxicity of spectinamides against J774 murine macrophages 54

Figure 4-1. In vitro $\mathrm{PK} / \mathrm{PD}$ system with two working units (Panel $\mathrm{A}$ ) and its different components (Panel B)

Figure 4-2. In vivo concentration-time profiles of QD regimens (Panel A), BID regimens (Panel B), and TID regimens (Panel C) simulated in the in vitro $\mathrm{PK} / \mathrm{PD}$ system

Figure 4-3. Relationship between cell counts and ATP present in a given sample..... 66 
Figure 4-4. Time-kill effect of Lee1445 against $M$. bovis BCG with observed log $\mathrm{CFU} / \mathrm{mL}$ plotted against time for different dosing regimens.................. 68

Figure 4-5. Model predicted profiles for various dosage regimens.......................... 71

Figure 4-6. $\quad$ PK/PD indices and their relationship to the microbial kill on day $6 \ldots \ldots . .72$

Figure 4-7. Pharmacokinetic profile of Lee1445 in rats following $20 \mathrm{mg} / \mathrm{Kg}$ IP injection................................................................................. 74

Figure 4-8. Numerical simulations for once daily dosing regimens of Lee1445 in

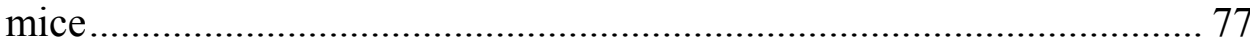

Figure 4-9. Numerical simulations for twice daily dosing regimens of Lee1445 in mice

Figure 4-10. Numerical simulations for thrice daily dosing regimens of Lee1445 in mice.

Figure 4-11. Simulated efficacy profiles of $200 \mathrm{mg} / \mathrm{Kg} /$ day administered as QD, BID and TID regimens.

Figure 4-12. Preliminary in vivo efficacy study of Lee1329 in the GKO mouse model where the dose selection criterion was arbitrary.

Figure 4-13. In vivo efficacy study of Lee1445 in the GKO mouse model where the dose selection criterion was based on PK/PD modeling approach 


\section{LIST OF ABBREVIATIONS}

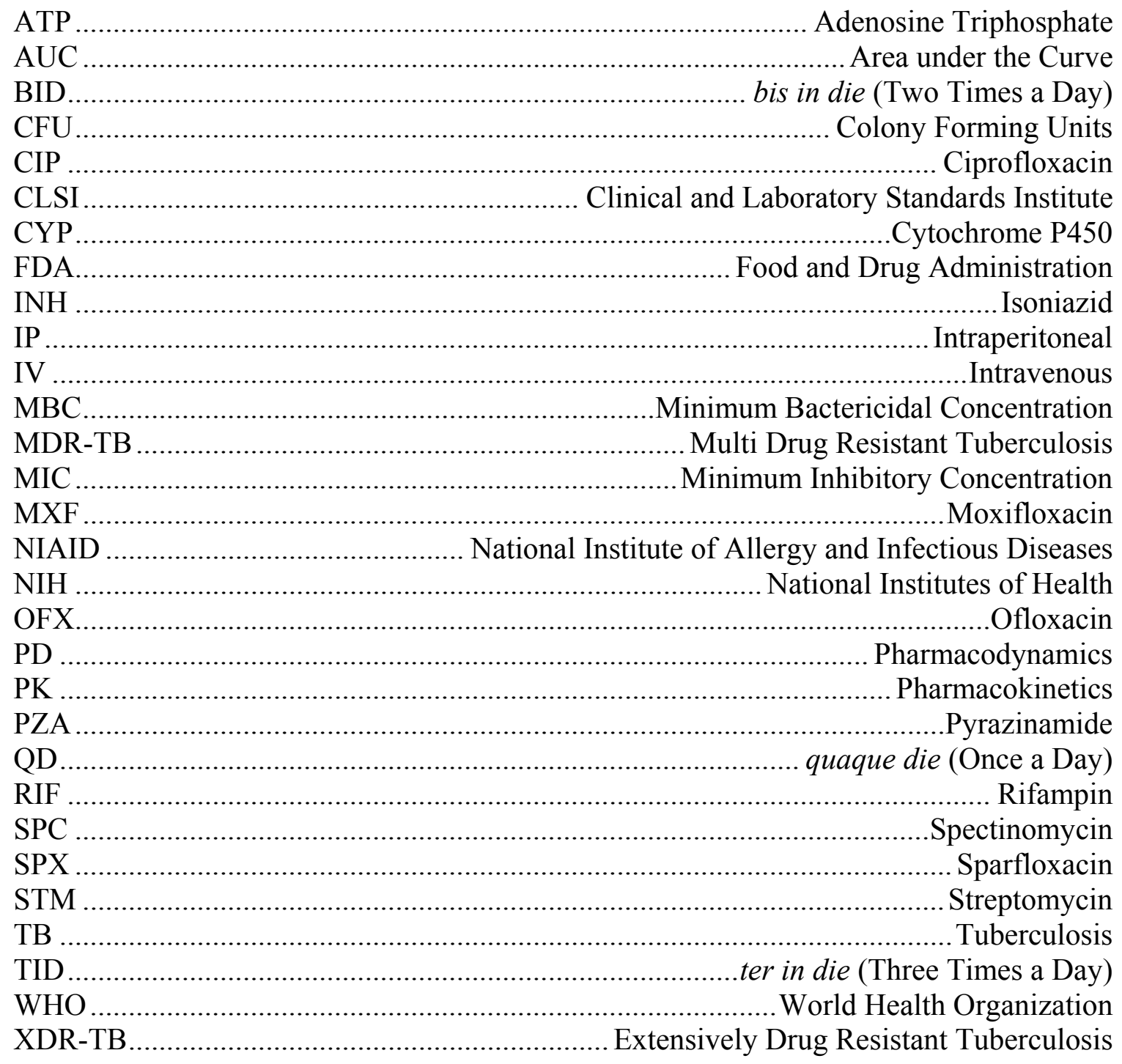




\section{CHAPTER 1. BACKGROUND*}

\section{Tuberculosis and Drug Development}

According to the World Health Organization (WHO) report on global tuberculosis control 2010, the incidence of tuberculosis (TB) cases was 9.4 million in 2009. There are half a million cases of multidrug resistant TB (MDR-TB) with approximately 58 countries and territories reporting at least one case of extensively drug resistant $\mathrm{TB}$ (XDR-TB) (1).

Currently used treatment strategies involve multiple drugs with treatment periods ranging from 6 months up to 2 years. MDR-TB patients in particular undergo treatment with more toxic and expensive drugs with poor patient compliance. Hence there is a definite need for more effective and better tolerated antituberculosis agents to go into optimized, less complicated and shorter dosage regimens. The path to new antibiotic drug discovery and development is a long and expensive process with very few compounds making it to the market. Development of antituberculosis drugs in particular was halted during the last decades of the $20^{\text {th }}$ century due to a lack of commercial incentive, which has only recently been addressed after a hiatus of almost 40 years through extensive governmental and non-governmental organizational and financial support.

In recent years the U.S. Food and Drug Administration (FDA) has suggested numerous approaches to optimize the drug development process. Pharmacokinetic/pharmacodynamic (PK/PD) modeling and simulation is one such innovative tool intended to help in early go or no-go decisions and significantly improving development efficiency. For example, in preclinical as well as clinical development, PK/PD modeling and simulation can be used to integrate independent measures of potency, safety, bioavailability, and clearance including their inter-individual variability and measurement uncertainty to develop optimal dosing regimens with regard to safety and efficacy, and to explore different treatment algorithms and trial designs with regard to their likelihood of being successful (2-4).

There is often only limited PK/PD information available for molecules under development because of a lack of inexpensive suitable animal models to predict efficacy, a poor understanding of the relationship between PK and PD for novel compounds, and challenges by complex disease processes, such as the phenomena of latency and drug tolerance in TB infections (5). Hence, improved tools are needed to evaluate the PK and PD properties and optimize dosing regimens for drug candidates early in the development process.

* This chapter adapted with permission. P.K. Vaddady, R.E. Lee, and B. Meibohm. In vitro pharmacokinetic/pharmacodynamic models in anti-infective drug development: Focus on TB. Future Med Chem., 2:1355-1369 (2010). 


\section{Pharmacokinetic/Pharmacodynamic Parameters of Antituberculosis Agents}

\section{Pharmacokinetics}

Pharmacokinetics describes the time course of concentration of a drug resulting from administration of a dosage regimen and accounts for its absorption, distribution, metabolism and excretion in the body. From a biopharmaceutic and pharmacokinetic standpoint, an ideal TB drug should possess good metabolic stability and high oral bioavailability, since oral administration is the route of choice for better patient compliance, indicating that the compound should have good aqueous solubility and gastrointestinal permeability. In order to allow lesser frequency of dosing, it should possess longer elimination half-life (5). To target the primary phase of TB infection, the drug should be able to penetrate into macrophages and also into the bacterial envelope assuming the drug target is located intracellularly.

\section{Metabolic Stability}

Drugs are most often eliminated in the liver via biotransformation and/or excretion into urine or bile. Along with xenobiotic transformation, liver plays a very important role in characterizing the metabolic stability, drug-drug interaction potential, and toxicity of drugs $(6,7)$. Two major enzyme systems Phase I and Phase II are responsible for biotransformation in the liver. Phase I reactions include oxidation, reduction and/or hydrolysis many of which are catalyzed by the cytochrome P450 (CYP) family of enzymes present in the smooth endoplasmic reticulum and require NADPH as a cofactor. Phase II enzymes present in the cytoplasm and endoplasmic reticulum are responsible for inactivating the drug via conjugation reactions with glucuronic acid, sulphate, glutathione and glutamine where they render the conjugate water soluble leading to its elimination. Sub cellular fractions of liver tissue known as microsomes are membrane vesicles of the smooth endoplasmic reticulum containing the CYP enzymes which can be used to assess the susceptibility of drug candidates to solely metabolism by CYP enzymes. Metabolic stability of a compound is investigated using such microsomes to determine metabolic half-life and intrinsic clearance which can be major determinants for in vivo hepatic clearance which further governs the pharmacokinetic properties such as first-pass elimination of the drug and there by oral bioavailability, systemic elimination and the half-life $(8,9)$.

\section{Protein Binding}

Free or unbound drug concentrations are of therapeutic interest because they are relevant for the pharmacological effect of a drug as well as its metabolism and excretion. Therefore it is important to estimate the extent of protein binding. Drugs are classified as highly bound if the free fraction in plasma $\left(f_{\text {up }}\right) \leq 0.1$, moderately protein bound if $0.1 \leq$ $f_{\text {up }} \leq 0.4$ and low protein bound if $f_{\text {up }} \geq 0.4$. Using the total instead of free drug concentrations becomes valid as long as the degree of binding and thus the ratio of free to total drug concentration remain constant. However if this ratio changes due to disease 
states or drug-drug interactions then it is important to note that the total drug concentration does not provide a valid substitute for free drug concentration. For drugs with low protein binding, protein binding modifications due to pathophysiological conditions or drug-drug interactions is not considered clinically relevant.

\section{Bioavailability}

Bioavailability is defined as rate and extent to which a drug is absorbed and becomes available in the systemic circulation. The three important components namely fraction absorbed from the gastrointestinal tract, fraction escaping the gut wall metabolism and the fraction escaping the first pass-metabolism contribute to the oral bioavailability. Physicochemical properties such as solubility, lipophilicity, charge state (extent of ionization) and stability, and physiological conditions such as gastric emptying time, intestinal transit time and gastrointestinal $\mathrm{pH}$ influence the absorption of drug molecules across the gastrointestinal mucosa (5).

Optimizing the chemical structure to improve one or more of these properties during the early discovery and development stage has been one of the approaches to improve oral bioavailability. Prodrug strategies have also been successfully implemented to improve the oral bioavailability of numerous compounds. In many cases, this involves masking a polar group by esterification to increase lipophilicity and enhance the extent of absorption from the gastrointestinal tract. After absorption, the ester is enzymatically hydrolyzed to release the parent drug (10).

\section{Drug Uptake into Macrophages and Mycobacteria}

MTB is a highly successful pathogen that parasitizes macrophages of the host by manipulating the phagosome that it resides in and prevents the normal maturation of this organelle into an acidic, hydrolytic compartment (11). A beneficial consequence of the accumulation of a drug within such host cells as macrophages is its increased activity against intracellular pathogens and therefore evaluating the drug uptake into macrophages plays an important role in the early drug development especially for antituberculosis

agents. Also by comparing the uptake of drugs, which do not possess any antituberculosis activity, into mycobacteria against those that are active, helps in the answering the fundamental mechanisms by which these compounds find their way across the prokaryotic cell envelope to their ribosomal targets.

\section{Pharmacodynamics}

Pharmacodynamics describes the intensity of drug effect in relation to its concentration. In the context of anti-infective therapy, pharmacodynamics defines the effect of drug on the pathogen residing in the host organism. In order to quantify the antituberculosis activity of drugs against the infectious pathogen, minimum inhibitory concentration (MIC) and minimum bactericidal concentration (MBC) are most commonly used parameters to date. 
MIC is defined as the lowest concentration that prevents growth of bacteria within a defined time period of incubation, typically around seven days for Mycobacterium tuberculosis (MTB) with a standard inoculum $\left(\sim 10^{5}\right.$ colony forming units $\left.(\mathrm{CFU}) / \mathrm{mL}\right)$ (12). MBC is defined as the drug concentration that produces $99.99 \%$ killing of bacterial cells in the initial inoculum. Although MIC and MBC seem to be good measures of the potency of a drug and its interaction with a pathogen, they do not provide any information on the time course of the antimicrobial activity.

$\mathrm{PK} / \mathrm{PD}$ indices are composites of a pharmacokinetic parameter such as area under the plasma concentration-time curve (AUC) or maximum plasma concentration $\left(\mathrm{C}_{\max }\right)$ and a microbiological parameter such as minimum inhibitory concentration (MIC). Most frequently used PK/PD indices of antibiotics based on MIC, shown in Figure 1-1, are time above MIC $(\mathrm{T}>\mathrm{MIC})$, ratio of peak concentration to $\mathrm{MIC}\left(\mathrm{C}_{\max } / \mathrm{MIC}\right)$, ratio of area under the curve (AUC) to MIC (AUC/MIC) and area under the inhibitory curve (AUIC), which is calculated as the area under the curve where the plasma concentration exceeds $\mathrm{MIC}\left(\mathrm{AUC}_{\mathrm{T}>\mathrm{MIC}} / \mathrm{MIC}\right)$. If drug concentrations remain above MIC at all times, then AUIC and AUC/MIC are identical (13).

The most common approach to antibiotic dosing is to adjust the doses to obtain antibiotic plasma concentrations that are above the MIC or a multiple thereof for a given pathogen throughout the dosing interval. Two distinct groups can be differentiated on the basis of the pattern of antimicrobial activity. The first pattern is characterized by concentration-dependent killing over a wide range of concentrations, where higher concentrations lead to a greater rate and extent of killing. Intracellularly acting drugs such as aminoglycosides, rifamycins and fluoroquinolones fall under this pattern. The second group involves time-dependent killing, where the time of exposure governs the bactericidal activity. Antibiotics that act on cell wall targets such as penicillins and cephalosporins exhibit such killing pattern (14).

AUC/MIC and $\mathrm{C}_{\max } / \mathrm{MIC}$ explain the bactericidal activity of concentration dependent killing while $\mathrm{T}>\mathrm{MIC}$ explains the time-dependent killing pattern. Table 1-1 provides a compilation of the $\mathrm{PK} / \mathrm{PD}$ indices and related $\mathrm{PK}$ parameters of existing antituberculosis drugs. It should be noted that, with the exception of the first-line agents for which some dose optimization in the TB indication has been performed, the calculated $\mathrm{PK} / \mathrm{PD}$ parameters indicated in the table may not necessarily be the most optimal for driving efficacy in TB patients.

\section{Spectinomycin}

Spectinomycin (SPC) is a broad-spectrum antibiotic that selectively targets bacterial ribosomes and remains important in both clinical and veterinary use (15). It was

first isolated in 1960 from the fermentation broth of Streptomyces spectabilis, a soil microorganism, by Upjohn research laboratory $(16,17)$. 


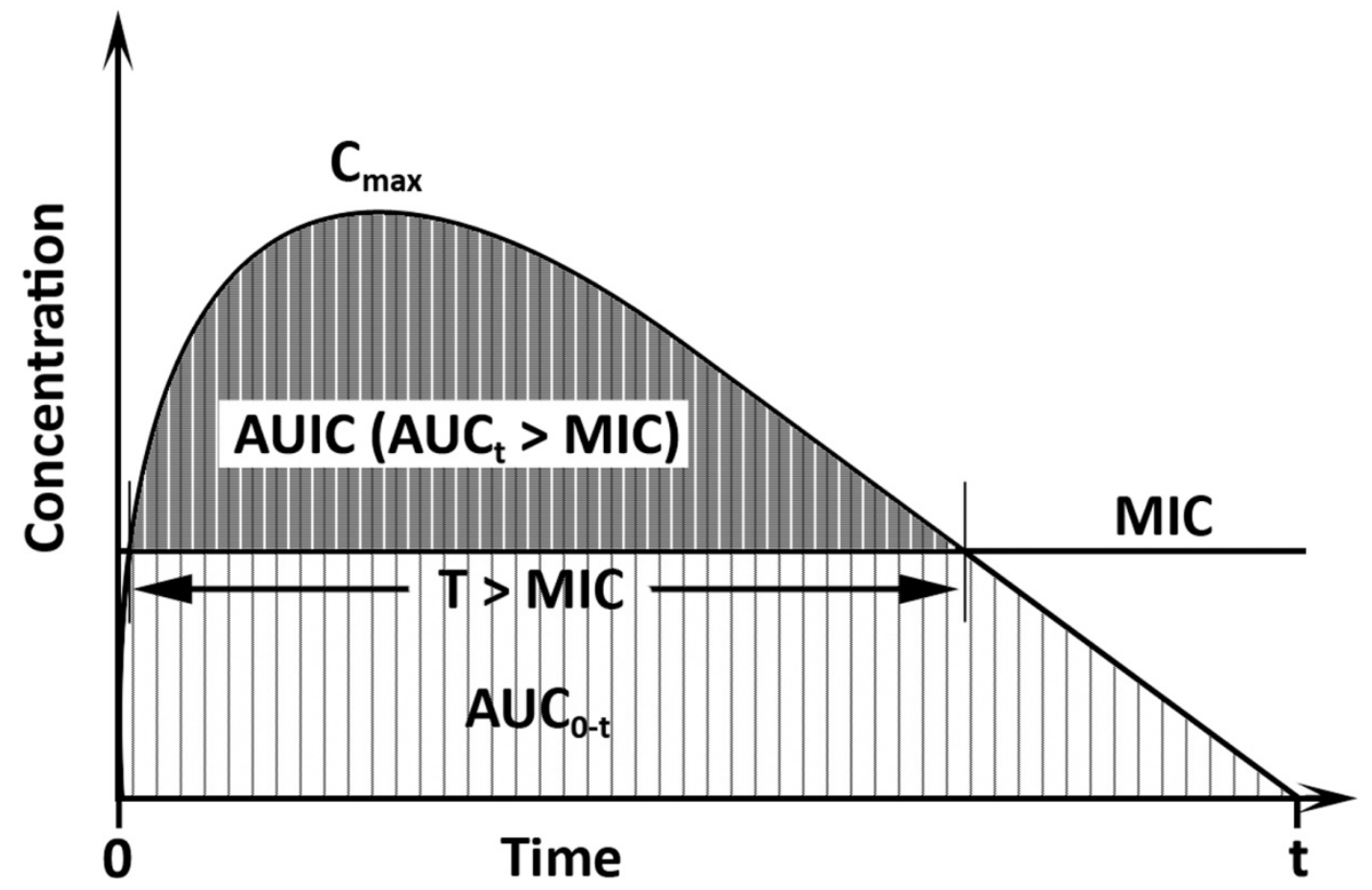

Figure 1-1. Commonly used pharmacokinetic/pharmacodynamic indices. 
Table 1-1. Reported PK/PD parameters of antituberculosis drugs (5, 18-27).

\begin{tabular}{|c|c|c|c|c|c|c|c|c|}
\hline Use & Class & Name & Dose range & Half-life (hr) & fup & $\mathrm{C}_{\max } / \mathrm{MIC}$ & AUC/MIC & $\mathrm{T}>\mathrm{MIC}$ \\
\hline \multirow[t]{6}{*}{ First-line } & Oral agents & Rifampin & $8-12 \mathrm{mg} / \mathrm{Kg}$ & $2-5$ & 0.1 & 24 & 39.9 & 9 \\
\hline & & Isoniazid & $4-6 \mathrm{mo} / \mathrm{Kg}$ & 2-4.5 (slow) & 0.99 & 40 & 19.2 & 18 \\
\hline & & & & $0.75-1.8$ (fast) & 0.99 & 40 & 11.6 & 9 \\
\hline & & Pyrazinamide & $20-30 \mathrm{mg} / \mathrm{Kg}$ & $10-24$ & 0.9 & 3.8 & 52 & na \\
\hline & & Ethambutol & $15-20 \mathrm{mg} / \mathrm{Kg}$ & $3-4$ & 0.7 & 10 & 23.4 & 13 \\
\hline & Injectables & Streptomycin & $12-18 \mathrm{mg} / \mathrm{Kg}$ & $2-4$ & 0.45 & 10 & 124.5 & 8 \\
\hline \multirow[t]{12}{*}{ Second-line } & Fluoroquinolones & Moxifloxacin & $400 \mathrm{mg}$ & $12-14$ & 0.5 & 12.3 & 110.5 & na \\
\hline & & Gatifloxacin & $400 \mathrm{mg}$ & $7-14$ & 0.8 & 9.5 & 85.6 & na \\
\hline & & Ciprofloxacin & $750 \mathrm{mg}$ & 4 & 0.6 & 5 & 16.9 & 10.5 \\
\hline & & Ofloxacin & $1000 \mathrm{mg}$ & $3-7$ & 0.7 & 5 & 47.4 & 15.5 \\
\hline & Injectables & Amikacin & $15 \mathrm{mg} / \mathrm{Kg}$ & $2-3$ & 0.9 & 43 & na & na \\
\hline & & Kanamycin & $15-30 \mathrm{mg} / \mathrm{Kg}$ & 2.5 & 0.99 & 20 & na & na \\
\hline & & Capreomycin & $15-30 \mathrm{mg} / \mathrm{Kg}$ & $4-6$ & na & 20 & na & na \\
\hline & Oral bacteriostatics & Ethionamide & $15-20 \mathrm{mg} / \mathrm{Kg}$ & $2-3$ & 0.7 & 1.6 & 1 & 1.5 \\
\hline & & Cycloserine & $15-20 \mathrm{mg} / \mathrm{Kg}$ & 10 & na & 3.8 & 195.5 & 22.5 \\
\hline & & PASA & $150 \mathrm{mg} / \mathrm{Kg}$ & $2-3$ & 0.85 & 75 & 153.7 & 4 \\
\hline & & Thiacetazone & $150 \mathrm{mg}$ & 4 & 0.05 & 1.3 & 1.2 & 5.5 \\
\hline & Others & Linezolid & $600 \mathrm{mg}$ & $4.5-5.5$ & 0.7 & 20 & 107.8 & na \\
\hline
\end{tabular}

Notes: PASA: Para-amino salicylic acid

fup: Fraction of unbound drug in plasma

Cmax/MIC: Ratio of peak serum concentration to minimum inhibitory concentration

AUC/MIC: Ratio of area under plasma concentration-time curve to MIC

T>MIC: Percentage of time during which the serum concentration remains above the MIC 


\section{Mechanism of Action and Indications}

SPC is an aminocyclitol antibiotic resembling kanamycin and gentamicin but is not an aminoglycoside (28-30). It binds to the $30 \mathrm{~S}$ ribosomal subunit of the microorganism and inhibits protein synthesis by interfering with peptidyl tRNA translocation and preventing elongation of the polypeptide chain at the translocation step (31). It sterically blocks the swiveling of the head domain of the small ribosomal subunit and thereby disrupts the translocation cycle (15).

Although it is active in vitro against a variety of aerobic gram-negative and grampositive bacteria, its primary clinical use has been in the treatment of uncomplicated infections with Neisseria gonorrhoeae. SPC is bactericidal against this pathogen, and most strains of gonococci are inhibited by $6.3 \mu \mathrm{g} / \mathrm{mL}$ or less of SPC with a range of 2 to $20 \mu \mathrm{g} / \mathrm{mL}$ (32). Trobicin $\AA$ (spectinomycin dihydrochloride pentahydrate) was first approved by Food and Drug Administration in 1971 for indications limited to acute gonococcal urethritis and proctitis in men and acute gonococcal cervicitis and proctitis in women (28). In veterinary medicine, it is used in many indications such as chronic respiratory disease, colibacillosis, paratyphoid, synovitis, and salmonella infections in chicken, bacterial enteritis in pigs and bacterial pneumonia in cattle (33).

\section{Preclinical and Veterinary Pharmacokinetics}

\section{Absorption}

SPC is rapidly absorbed following intramuscular (i.m.) administration. Following single i.m. administration of $50 \mathrm{mg} / \mathrm{Kg}$ in chicken, $\mathrm{C}_{\max }$ was found to be $152.8 \mu \mathrm{g} / \mathrm{mL}$ achieved at $0.25 \mathrm{hr}$. SPC was completely bioavailable following i.m. administration with absolute bioavailability $\left(\mathrm{F}_{\text {abs }}\right)$ values of $136 \%$ in chicken, $118 \%$ in cattle and $104 \%$ in sheep. Subcutaneous (s.c.) administration of $50 \mathrm{mg} / \mathrm{Kg}$ in chicken produced a $\mathrm{C}_{\max }$ of $99.8 \mu \mathrm{g} / \mathrm{mL}$ and $\mathrm{F}_{\text {abs }}$ of $128 \%$. However, following oral administration of $50 \mathrm{mg} / \mathrm{Kg}$ and $100 \mathrm{mg} / \mathrm{Kg}$ in chicken, $\mathrm{F}_{\text {abs }}$ was found to be 11.8 and $26.4 \%$ with $\mathrm{C}_{\max }$ values of 5.13 and $14.26 \mu \mathrm{g} / \mathrm{mL}$ respectively, indicating that SPC is poorly absorbed from the gastrointestinal tract (33-35). Repeated administration in cattle did not result in higher tissue concentration than those achieved via single dose $(33,36)$.

\section{Distribution}

After a single intravenous (i.v.) administration of $50 \mathrm{mg} / \mathrm{Kg}$ in chicken, the volume of distribution of SPC based on the terminal phase $\left(\mathrm{V}_{\mathrm{z}}\right)$ was $0.34 \mathrm{~L} / \mathrm{Kg}$ suggesting that the distribution of the drug is limited to extracellular fluid. Similar values have been reported for cow $(0.3 \mathrm{~L} / \mathrm{Kg})$ and sheep $(0.3 \mathrm{~L} / \mathrm{Kg})(34,37)$. Twelve hours following i.m. administration and 24 hours following oral administration, concentrations have been found in the following swine tissues in decreasing order: kidney, liver, lung, muscle and fat. A similar profile has been observed in cattle following i.m. administration after 24 and 72 hours. Tissue/serum ratios did not exceed 0.5 and were found to be much 
lower in brain, aqueous humor and bone (38). Protein binding was found to be low (10\%) in cows (37).

\section{Metabolism and Excretion}

SPC does not appear to undergo any significant metabolism. In swine it was found to be excreted unchanged in the urine following i.m. administration. Following single dose i.m. administration, the overall elimination half-life $\left(\mathrm{t}_{1 / 2 \beta}\right)$ of SPC was comparable across species with cattle $(1.2 \mathrm{hr})$, sheep $(1.1 \mathrm{hr})$, pigs $(0.98 \mathrm{hr})$ and chicken $(1.65 \mathrm{hr})$ respectively. This short elimination half-life suggests that SPC is eliminated rapidly from the body and supports the fact that a majority of it is excreted unchanged in the urine $(34,36,37,39)$.

SPC is rapidly absorbed and quickly eliminated from plasma as well as tissues via urinary elimination. This explains the reason for no accumulation following repeated doses in cattle. Since a majority of this drug is renally eliminated it is important to note that accumulation can occur in renally impaired animals. Following oral administration SPC is excreted mostly in the feces as it is poorly absorbed from the gastrointestinal tract. Total clearance in chicken has been reported as $2.68 \mathrm{~mL} / \mathrm{min} / \mathrm{Kg}$ following i.m. administration $(33,34,36,38)$.

\section{Human Pharmacokinetics}

\section{Absorption}

SPC is not significantly absorbed from the gastrointestinal tract but is well absorbed when given via the intramuscular route. Following a $0.5 \mathrm{~g}$ dose via i.m. injection, the absorption rate constant was found to be $1.48 \mathrm{hr}^{-1}$ corresponding to an absorption half-life of $0.47 \mathrm{hr}$. Following a single two-gram i.m. injection, peak serum concentrations average $100 \mu \mathrm{g} / \mathrm{mL}$ at one hour, while a single four-gram injection produces peak serum concentration averaging $160 \mu \mathrm{g} / \mathrm{mL}$ at two hours. Average serum concentrations of $15 \mu \mathrm{g} / \mathrm{mL}$ for the two-gram dose and $31 \mu \mathrm{g} / \mathrm{mL}$ for the four-gram dose were present eight hours after dosing (40-42). In children, serum concentrations ranged from 22 to $87.5 \mu \mathrm{g} / \mathrm{mL}$ following $40 \mathrm{mg} / \mathrm{Kg}$ i.m. administration (43).

\section{Distribution}

SPC when administered via i.v., i.m. or constant rate i.v. infusion shows a biexponential disposition with a volume of central compartment about twice the volume of the peripheral compartment. The average total volume of distribution was $11.5 \mathrm{~L}$. The average inter-compartmental distribution rate constant was found to be approximately 0.5 $\mathrm{hr}^{-1}$. SPC has minimal protein binding (42). Tissue distribution studies performed in uterus and fallopian tube suggested that tissue levels of SPC were about one-third to onequarter the levels in the serum (40). 


\section{Metabolism and Excretion}

SPC does not undergo significant metabolism. It is largely eliminated by renal excretion. The first order elimination rate constant following a single dose $0.5 \mathrm{~g}$ i.m. administration was $0.694 \mathrm{hr}^{-1}$ corresponding to a half-life of about $1 \mathrm{hr}$. Following i.v. and i.m. administration approximately $75 \%$ of the drug was excreted unchanged in the urine (42). The pharmacokinetics of SPC after $2 \mathrm{~g}$ i.v. injection studied in volunteers with varying degrees of renal function showed similar mean peak concentrations as compared to individuals with normal renal function but the half-lives ranged from 4.7 to $29.3 \mathrm{hr}$ (44). Since SPC is minimally bound to plasma proteins, the antibiotic may be excreted entirely by glomerular filtration and hence changes in renal function can significantly impact the clearance of the drug.

\section{Toxicity}

SPC is well tolerated when administered as single i.v. or i.m. injection. In animals, i.m. injection of large doses of SPC for 90 days produced only low-grade histopathologic changes at the injection sites (45). It does not show cross-allergenicity with penicillins and is relatively non-toxic. Experiments performed to elaborate immunogenic and anaphylactic properties of SPC showed no allergy in guinea pigs. The anaphylactic activity was found to be of very low order with no mast cell degranulating action (46).

Compared to the related aminoglycoside antibiotics, SPC appears to be neither ototoxic nor nephrotoxic (41). When administered at dosages up to $120 \mathrm{mg} / \mathrm{Kg}$ per day for 75-90 days, it showed no signs of cochlear or vestibular dysfunction in cats. When cattle were overdosed at $150 \mathrm{mg} / \mathrm{Kg} /$ day ( 10 times the labeled dose) for 5 days, there was increased relative kidney weight. Urinary $\mathrm{pH}$ decreased and squamous and transitional cells were found in the kidney (33). Clinical signs of ataxia and coma following a single $90 \mathrm{mg}$ s.c. injection were transient in turkey poults which resolved after 4 hours (38).

Clinical experience indicates good tolerance of single i.m. dose of SPC. Tolerance studies in 25 healthy male volunteers who were given i.v infusions of SPC in doses ranging from 0.5 to $8 \mathrm{~g}$ /day for 5 consecutive days showed no signs of otoxicity (audiometric examinations), hepatotoxicity (SGPT, total bilirubin and alkaline phosphatase), nephrotoxicity (BUN, chemical and microscopic urinalysis) or local intolerance (evaluation of infusion sites) during the treatment (47). In another study, volunteers who were on daily $8 \mathrm{~g}$ dose for a longer duration of 21 days did not develop cochlear, vestibular, or renal abnormalities (48). In the much smaller doses used to treat gonococcal infections, adverse reactions such as dizziness, urticaria, nausea, chills, fever, and insomnia have been uncommon and serious adverse reactions have not been reported $(41,49)$.

Comparison of the structural formula to aminoglycoside antibiotics suggests that SPC is a fused tricyclic molecule containing a unique aminocyclitol actinamin but not 
aminosugar and because of this unique difference, it is speculated that aminosugar portions and their position in the antibiotic determine the degree of toxicity (48).

\section{Streptomycin}

Streptomycin (STM) is an antibiotic produced from the actinobacterium Streptomyces griseus. It was the first drug in the class of aminoglycosides and the first antibiotic that was effective in treating TB. It was originally discovered in 1944 by Schatz, Bugie and Waksman when they demonstrated its inhibitory action in vitro against tubercle bacilli $(50,51)$.

\section{Mechanism of Action and Indications}

STM is a bactericidal antibiotic that acts by interfering with normal protein synthesis. Its site of action is at the ribosomal protein $\mathrm{S} 12$ ( rpsL) of the $30 \mathrm{~S}$ subunit of the ribosome, and 16S rRNA (rrs) in the protein synthesis (52).

Streptomycin is suggested in the therapy of tuberculosis, brucellosis, tularemia and yersinia infections, several of these require the co-administration of another agent (53). It is one of the first drugs that was active against Mycobacterium tuberculosis and is currently used as a second line agent against MTB when one or more of the first line agents are contraindicated because of toxicity, intolerance or resistance. STM is also shown to be efficacious in the treatment of non-tuberculosis infections such as plague, tularemia, influenza (respiratory, endocardial, and meningeal infections), pneumonia, urinary tract infections, and gram negative bacteremia.

\section{Preclinical and Veterinary Pharmacokinetics}

\section{Absorption}

STM is rapidly absorbed via the intramuscular route. Following i.m. administration of dihydro streptomycin (DSTM), a hydrogenated form of streptomycin, at a dose of $10 \mathrm{mg} / \mathrm{Kg}$ once daily for three days in cattle and sheep, mean $\mathrm{T}_{\max }$ was found to be about at $1.4 \mathrm{hr}$ and $1.7 \mathrm{hr}$ respectively, with average peak serum concentrations of approximately $23 \mathrm{mg} / \mathrm{L}$ (54). In dogs and swine, $\mathrm{T}_{\max }$ was found to be $60 \mathrm{~min}$ with an absorption half-life in swine of $14 \min (55,56)$. In a study performed in camels, following $10 \mathrm{mg} / \mathrm{Kg}$ i.m. administration of STM, the absorption half-life was found to be 10.8 min with a mean peak serum concentration of $34.4 \mathrm{mg} / \mathrm{L}$ achieved $30 \mathrm{~min}$ after drug administration. Absolute bioavailability in camels via this route was found to be $98 \%$ (57). 


\section{Distribution}

STM administered via the i.v. and i.m. routes shows a bi-exponential disposition with the apparent volume of distribution numerically similar to the extracellular fluid volume. In a study performed to evaluate the pharmacokinetics of STM after i.v. and i.m. administration in horses, $\mathrm{Vd}$ was found to be $230 \mathrm{~mL} / \mathrm{Kg}(58)$. In a similar study conducted in camels, mean Vd estimated by the area method was found to be $265 \mathrm{~mL} / \mathrm{Kg}$ (57), where the extra cellular fluid volume in camels has been estimated to be $200 \mathrm{~mL} / \mathrm{Kg}$ (59). In cattle, sheep and pigs Vd was found to be $0.39,0.31$ and $0.4 \mathrm{~L} / \mathrm{Kg}$ respectively.

\section{Metabolism and Excretion}

STM does not undergo significant metabolism. Approximately two thirds of the administered dose is recovered in urine after 24 hours. The distribution half-life $\left(\mathrm{t}_{1 / 2 \alpha}\right)$ in cattle was $0.71 \mathrm{hr}$ and the elimination half-life $\left(\mathrm{t}_{1 / 2 \beta}\right)$ was $4 \mathrm{hr}$ after a single $10 \mathrm{mg} / \mathrm{Kg}$ i.m. injection of STM (60). The $\mathrm{t}_{1 / 2 \beta}$ of STM in horses and camels was $3.4 \mathrm{hr}$ and $4.7 \mathrm{hr}$ respectively $(57,58)$ compared to $2-3 \mathrm{hr}$ in humans $(61)$. Urine $\mathrm{pH}$ in herbivores is alkaline whereas in humans it is more acidic and STM being a basic drug tends to be more unionized in alkaline $\mathrm{pH}$ leading to greater reabsorption from kidney tubules accounting for longer plasma half-lives in cattle, horses and camels (60). The mean systemic clearance of STM in horses, camels and cattle was found to be $0.79,0.93$ and $1.58 \mathrm{~mL} / \mathrm{min} / \mathrm{Kg}$ respectively $(57,58,60)$. In one of the studies of STM at $10 \mathrm{mg} / \mathrm{Kg}$ administered intramuscularly in lactating buffaloes, unchanged drug appeared in milk at 3 hours and remained up to 8 hours with concentrations varying from 0.07 to $0.45 \mathrm{mg} / \mathrm{L}$ (60).

\section{Human Pharmacokinetics}

\section{Absorption}

STM because of its poly-cationic nature is not absorbed following oral administration and hence parenteral injection is the usual method of administration, the i.m. route being the most common. A $1000 \mathrm{mg}$ single daily dose (QD) or $500 \mathrm{mg}$ twice daily dose (BID) results in peak serum levels of about $50-60 \mathrm{mg} / \mathrm{L}$ and $15-30 \mathrm{mg} / \mathrm{L}$ respectively within one hour of intramuscular administration (62). Oral administration of $1 \mathrm{~g}$ daily dose does not produce significant concentrations in the blood and about 72 to $110 \%$ of the daily dose could be detected in the feces. When STM is administered intrathecally there is a good retention of the drug in cerebrospinal fluid (CSF). A dose of $15-20 \mathrm{mg}$ results in concentrations from 13 to $25 \mathrm{mg} / \mathrm{L}$ of CSF after 24 hours (63). Continuous i.v. infusion of $3 \mathrm{~g} /$ day produces concentrations in the blood varying between 20 to $60 \mathrm{mg} / \mathrm{L}(64)$. 


\section{Distribution}

STM is distributed into body tissues such as kidney, bile, lungs, heart and muscle, and fluids such as peritoneal, ascetic, synovial, intraocular and pleural fluid except for CSF. It passes through the placental barrier into the fetal blood and is also secreted into milk, saliva and sweat (64). About $30 \%$ of the drug is protein bound $(65,66)$. The volume of distribution is approximately $0.25 \mathrm{~L} / \mathrm{Kg}(62)$.

\section{Metabolism and Excretion}

Approximately $50-60 \%$ of parenterally administered STM dose is excreted unchanged in the urine within first the 24 hours and total urinary excretion accounts for $70-90 \%$ of the total dose in patients with normal renal function which is apparently accomplished by glomerular filtration (67). No metabolites have been identified for STM. About $2-5 \%$ of the drug is excreted in the bile and approximately $2 \%$ of the dose administered parenterally can be recovered from the feces which represents biliary rather than bowel excretion (64). The half-life of STM in humans is about 2-3 hours with clearance of $70 \mathrm{ml} / \mathrm{min} / \mathrm{m}^{2}$ (65). Since STM has a low plasma protein binding and is excreted entirely by glomerular filtration, changes in renal function can significantly impact the clearance of the drug and hence dosage adjustments are needed in renally impaired patients.

\section{Toxicity}

STM adversely affects auditory, vestibular, and renal function in both animals and humans. Its toxic effects can be separated into acute and chronic effects. Acute effects are usually reversible and include neuromuscular blockade and acute hearing impairment. Chronic effects pose a major clinical problem and usually cause permanent damage to the individual. They include damage to the kidney and the inner ear $(68,69)$.

\section{Nephrotoxicity}

The proximal renal tubule is the major site of damage exerted by STM. Although the exact chain of events in STM nephrotoxicity is unclear, accumulation of drug and phospholipids within lysosomes is involved. The lysosomes become overloaded with phospholipid, destabilize and rupture leading to release of acid hydrolases and of high concentrations of aminoglycoside into the cytoplasm where they disrupt cell structure and function (70). In a study, where a total dosage of $75 \mathrm{mg} / \mathrm{Kg} /$ week of STM was given either as a $30 \mathrm{~min}$ i.v. infusion administered daily ( $15 \mathrm{mg} / \mathrm{Kg}$ given Monday to Friday) or 3 times weekly (25 mg/Kg administered Monday, Wednesday, and Friday), 19\% of the patients developed nephrotoxicity, which was defined as an increase in the serum creatinine level of $>0.5 \mathrm{mg} / \mathrm{dL}$ for patients with baseline serum creatinine levels between $0.5-1.9 \mathrm{mg} / \mathrm{dL}$ (71). Prolonged duration of therapy, greater total dose, hypotension, volume depletion, high peak or trough serum concentrations, concurrent 
liver disease, and concurrent use of other nephrotoxic drugs are shown to be risk factors pertaining to STM ototoxicity (72-74).

\section{Ototoxicity}

Ototoxicity is characterized by an early symptom tinnitus. Discontinuance of the drug at this stage can probably prevent auditory loss. Hearing loss occurs as a result of degeneration of the hair cells of the cochlear, beginning at the basal coil and progressing to the apex. Loss starts with high frequencies followed by loss of lower frequencies (75). Within the vestibular apparatus, hair cell damage starts in the apex of the cristae and the striolar regions of the maculae and progresses towards the periphery of the vestibular receptor (76). Along with the sensory cells, afferent nerve endings deteriorate. STM predominantly causes vestibulotoxicity whose symptoms include disequilibrium and ataxia (77). Proposed risk factors include genetic predisposition and total dose administered $(78,79)$. The onset occurs approximately at the end of the fourth week of therapy with daily doses ranging from 1 to $2 \mathrm{~g}$ and about the third week with higher doses of $3 \mathrm{~g}$ daily (80).

\section{Classification of In Vitro PK/PD Models}

In vitro $\mathrm{PK} / \mathrm{PD}$ models have many favorable characteristics such as flexibility, adaptability, relatively low cost, good correlation with human and animal data, and no ethical concerns as compared to animal experiments which make them excellent experimental platforms (81). Especially with antituberculosis drugs, the drugmycobacterial interaction can be measured more precisely than in vivo since the influence of the immune system is absent in such models. On the basis of drug exposure and bacterial concentration these in vitro models can be classified into static models and dynamic models.

\section{Static Models}

As the name suggests, static models evaluate how a bacterial culture responds to a constant environment with a fixed antibiotic exposure. Since there is no exchange of medium in these systems and bacteria utilize the same medium during the course of the experiment, their growth is limited by nutrition, space, aeration and toxic metabolites in addition to the effect of the antibiotic (13). However, MIC determined from such a setup is the most prevalent and best understood in vitro PD parameter for antibiotics, which is used routinely to track antibacterial resistance in clinical isolates (82). Although these models have been extensively used (83-87), they cannot provide information on the time course of antimicrobial activity under varying antibiotic concentrations as observed in vivo and since MIC determination depends on the number of bacteria at a given time point, many different combinations of growth and kill rates can result in the same MIC (13). However, these models when studied with a range of constant antibiotic concentrations over a period of time can provide useful information on the kill kinetics, 
which approximates a constant infusion scenario of an intravenous antibacterial agent in vivo.

The application of such models for studying the PK/PD relationships of the antituberculosis agents rifampin (RIF), isoniazid (INH) and fluoroquinolones such as moxifloxacin (MXF), ofloxacin (OFX), sparfloxacin (SPX), and ciprofloxacin (CIP) has been reported by Jayaram et al. (88-90), and is presented in panel A of Figure 1-2. The kill kinetics were determined in BACTEC 7H12B medium, followed by plating on 7H11 agar plates to obtain colony counts. The MTB culture was inoculated into BACTEC vials which were monitored daily by a radiometric detection method using the BACTEC TB 460 system, in which the mycobacterial growth is determined by the utilization of ${ }^{14} \mathrm{C}$ with release of ${ }^{14} \mathrm{CO}_{2}$ by the multiplying bacteria. Fixed drug concentrations ranging from high to low were added to the vials and samples from the cultures with each concentration along with a drug free control were plated over 9 days to determine the $\mathrm{CFU} / \mathrm{mL}$. Time-kill curves were plotted and analyzed for the rate and extent of bacterial killing. Rate of killing was determined from the start of experiment to the time of maximal reduction in the $\log _{10} \mathrm{CFU} / \mathrm{mL}$ and in vitro dose response curves were obtained by plotting the $\log _{10} \mathrm{CFU} / \mathrm{mL}$ against the ratio of the concentration to its MIC in broth $\left(\mathrm{C}_{\text {broth }} / \mathrm{MIC}\right)$. Each fixed concentration of the drug was multiplied by the time of exposure to get AUC which was then divided by MIC in broth to obtain the AUC/MIC index. The results from these studies allowed the authors to quantify PD parameters that could describe the in vitro bactericidal efficacy and indicated AUC-dependent killing for RIF, concentration-dependent killing for INH, and a time-dependent killing for fluoroquinolones.

\section{Dynamic Models}

Any typical dosage regimen for antituberculosis drugs involves periodic administration of the antibiotic with varying drug concentrations at the effect site due to in vivo absorption, distribution and particularly clearance mechanisms. As bacteria react to these changing concentrations differently than to exposure to constant concentrations, it is important to mimic similar conditions in vitro to understand the rate and extent of bacterial kill by the respective antibiotic under these conditions. Dynamic models utilize time-kill curves, which follow the microbial killing and growth as a function of both time as well as antibiotic concentration. These dynamic models used to study the PK/PD of antituberculosis agents can be further differentiated as follows:

Type I: Models without Filters Involving Bacterial Loss, Where the Mechanism of Drug Loss Involves Dilution

In order to simulate in vivo drug clearance, a systematic loss of drug from the model becomes essential. This was usually achieved in early models via dilution performed in a stepwise or continuous manner. To a culture vessel containing actively growing bacteria, a known volume of culture was either substituted with fresh medium lacking the antibiotic at fixed intervals or it was infused using a peristaltic pump where 


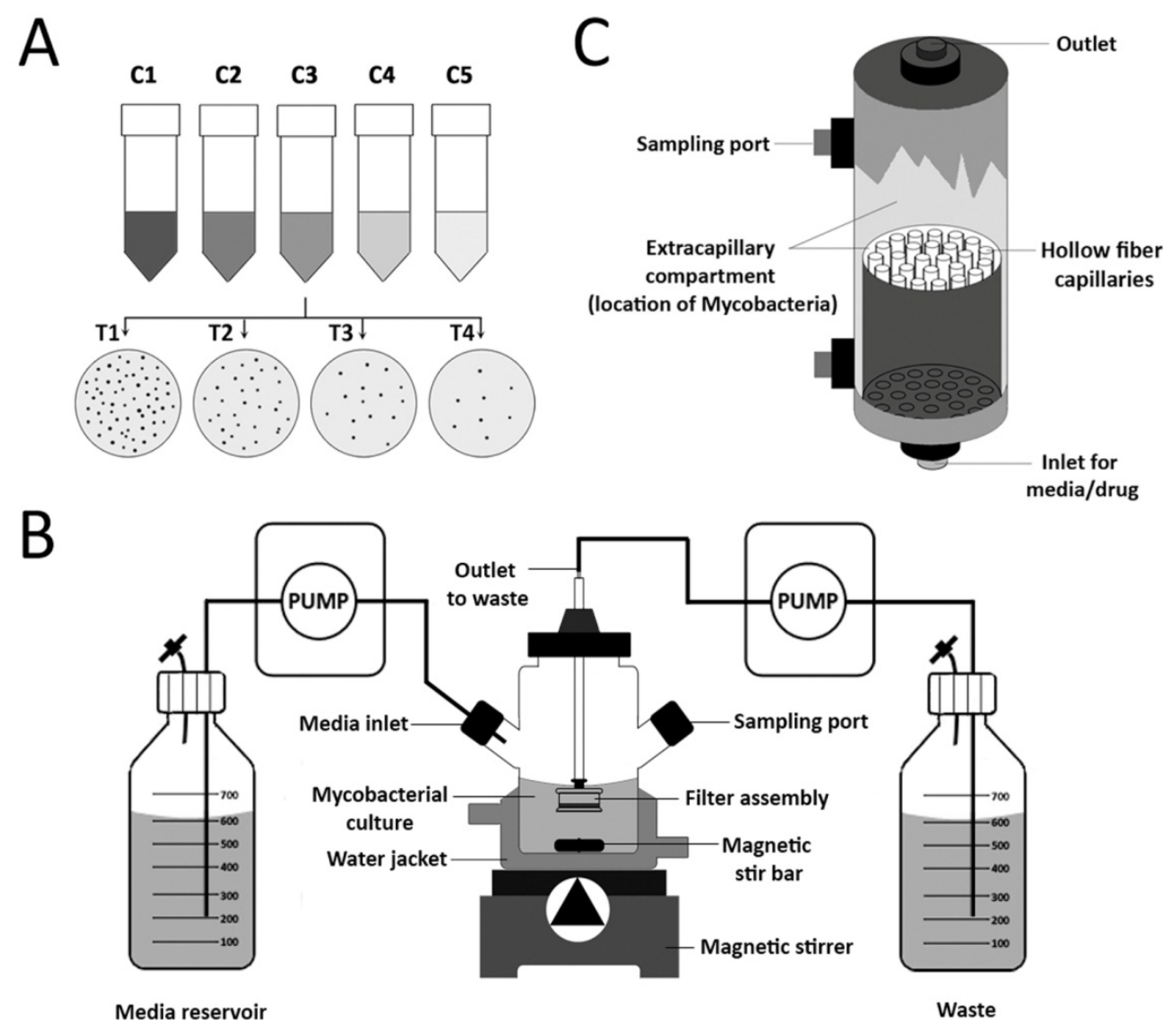

Figure 1-2. In vitro $\mathrm{PK} / \mathrm{PD}$ models for antituberculosis agents. Panel A shows a static model where $\mathrm{C} 1$ to $\mathrm{C} 5$ denote a series of concentrations of a drug and $\mathrm{T} 1$ to $\mathrm{T} 4$ denote times at which aliquots of mycobacteria are plated for every given concentration of drug. Panel B shows a dynamic model with filter assembly involving no bacterial loss and the mechanism of drug loss involves simple dilution by the media. Panel $\mathrm{C}$ shows a hollow fiber model where there is no bacterial loss and the mechanism of drug loss involves diffusion into extra capillary compartment. 
the excess was directed into waste. Although these models mimicked a one compartment pharmacokinetic model, they suffered from dilutional loss of bacteria $(91,92)$. Loss of bacteria from the model leads to a bias which can be corrected using mathematical equations $(93,94)$. However, for slow growing organisms, the resulting bias may be substantial and inadequate for mathematical correction. Therefore no such models have been reported so far for studying antituberculosis agents.

Type II: Models with Filters Involving No Bacterial Loss, Where the Mechanism of Drug Loss Involves Dilution

The use of filters or membranes which do not allow the bacteria to be washed out are alternative approaches to overcome the issue of bacterial loss. A modified version of type I models involving a filter to prevent bacterial loss and a stirrer to prevent blockage of the filter membrane and to maintain the homogeneity of the culture has become popular to study the effects of antibiotics. One such model to evaluate time-kill curves of antituberculosis drugs against mycobacteria has recently been reported by Budha et al. (19).

The in vitro system, as shown in panel B of Figure 1-2, consisted of a two-armed, water jacketed spinner culture flask, where a filter unit, consisting of a pre-filter $(5 \mu \mathrm{m})$ and filter membrane $(0.22 \mu \mathrm{m})$, was used to prevent leakage of bacteria during the dilution process. One of the arms covered with silicone septa was used for dosing and repeated sampling. The other arm was connected to a reservoir containing antibiotic free sterile medium. The whole filter unit was suspended into the media from the top via a hollow steel tube whose outlet was connected to a peristaltic pump to continuously withdraw the medium at a constant rate. The flask was placed on a magnetic stirrer, which ensured homogeneity of the culture and also prevented membrane pore blockage. The temperature in the flask was maintained at $37^{\circ} \mathrm{C}$ for bacterial growth by attaching a thermostatic water circulator to the water jacket of the flask. In this model, mycobacteria were exposed to INH concentration-time profiles as encountered during multiple dose regimens with 25, 100 and $300 \mathrm{mg}$ /day in humans who are either fast or slow INH metabolizers or the resulting time-kill curves were obtained. The different elimination half-lives $\left(\mathrm{t}_{1 / 2}\right)$ of INH in slow vs. fast metabolizers were simulated in the model by varying the flow rates of the medium. The results from this study confirm that $\mathrm{AUC}_{0-24} /$ $\mathrm{MIC}$ is the most explanatory PK/PD index for the antimicrobial effect of INH.

\section{Type III: Hollow Fiber Models Involving No Bacterial Loss, Where the Mechanism of} Drug Loss Involves Diffusion

In spite of the modifications in type II models, membrane pore blockage could be a problem which may become more pronounced when antibiotics with short half-lives are studied, since the flow rate across the filter is higher (95). In order to overcome this problem, a diffusion or dialysis of the drug to create the desired antibiotic concentration profile in a separate bacterial compartment has been employed by several groups (96-98). 
Gumbo et al. (99-104) published several studies of the antituberculosis agents RIF, INH, MXF and pyrazinamide (PZA) utilizing a hollow fiber bioreactor system, which is an example of a diffusion model. Hollow fiber systems represent a two compartment model. The central compartment of the hollow fiber system is composed of the central reservoir, the inner lumina of the hollow fiber capillaries, and the oxygenpermeable flow path connecting the central reservoir to and from the hollow fibers. The peripheral compartment is the space outside the hollow-fiber capillaries that is enclosed by an impermeable plastic encasement. The hollow fiber bioreactor system allows MTB to grow in the peripheral compartment of a hollow fiber cartridge. This peripheral compartment is separated from the central compartment by semi-permeable hollow fibers, with pore sizes that selectively allow transfer of nutrients, drugs and bacterial metabolites but restrict bacteria from leaving the peripheral compartment, as shown in panel $\mathrm{C}$ of Figure 1-2. In these experimental systems, computer controlled peristaltic pumps are used to administer drugs via a dosing port and fresh $7 \mathrm{H} 9$ broth is pumped into the afferent port of the central compartment of the hollow fiber system, while drugcontaining media is iso-volumetrically removed from the efferent port of the system at rates programmed to simulate the drug half-life encountered in humans.

These in vitro models were used to study a wide variety of issues such as selection of MXF doses that suppress drug resistance (99), to understand reasons for failure of CIP (100) and INH treatment (101), to predict efficacy of INH in different ethnic populations (102), and to evaluate the PK/PD parameters of RIF (103) and PZA (104). Dose-effect and dose-scheduling studies were also performed with ethambutol to identify exposures and schedules linked to optimal kill and resistance suppression (105). In spite of these advancements, there are limitations associated with the use of the hollow fiber bioreactor models. These bioreactors are complex and difficult to sterilize between experiments, and hence new hollow fiber cartridges are recommended for every study.

\section{Perspectives Relevant to Drug Development}

The use of in vitro models in drug development has increased tremendously in the recent past. Several scenarios for evaluating the activity of new molecules can be studied using these models MIC does not provide any information on the persistence of antibiotic effects. Such inhibitory effects that persist after exposure to an antibiotic can be studied in these models. Also, antituberculosis therapy is a combination of several antibiotic agents which is further complicated when treating patients co-infected with HIV, and it is challenging to study the different drug combinations in vivo from a drug development perspective.

Whole blood bactericidal assays, serial sputum colony counting and liquid culture have been used in Phase II studies to look at different combinations of drugs albeit not exhaustively and only with specific regimens of particular interest. In vitro models are reasonably simple to quantitatively assess the efficacy of such combinations and to perform more comprehensive experiments addressing these questions. Since it is relatively easy to study antimicrobial activity of drugs against different strains of bacteria 
in these systems, these models may also serve as useful tools to study the emergence of resistance and help identify novel drugs or combinations thereof to treat drug resistant strains.

Owing to the fact that these in vitro $\mathrm{PK} / \mathrm{PD}$ techniques are relatively new in the area of antituberculosis drug development and tedious to work with because of the long doubling times of mycobacteria, only limited or no studies have been reported pertaining to the following three sections. Hence we provide an insight into how these scenarios can be explored in the future using such in vitro models.

\section{Postantibiotic Effect}

The rate of bacterial killing and time before re-growth of surviving bacteria influences the design of an optimal dosing interval. Postantibiotic effect (PAE) refers to the continued suppression of bacterial growth following limited exposure of organism to an antibacterial agent (106). It can be demonstrated in vitro by observing bacterial growth after a drug is removed. The standard method to quantitate PAE is to calculate the difference in time required for drug-exposed and control cultures to increase one $\log _{10}$ above the number present immediately after withdrawal of the antibiotic (107). The PAE using bacterial counts as a parameter is calculated by $\mathrm{PAE}=\mathrm{T}-\mathrm{C}$, where $\mathrm{T}$ is the time required for bacterial counts of drug-exposed cultures to increase one $\log 10$ above the counts observed immediately after washing/dilution and $\mathrm{C}$ is the corresponding time required for counts of untreated cultures (108).

PAE has been used to determine the optimal interval between dosing and is the basis of twice or thrice weekly therapy for TB. Administration of drugs at less frequent dosing intervals may not only improve patient compliance but may also reduce toxicity and costs involved in the treatment. Studies performed using static models with INH, RIF, Streptomycin (SM), Ethambutol (EMB), Rifapentine and MXF have each demonstrated postantibiotic effects against MTB (109, 110). Prolonged PAEs are usually observed with antibiotics that are inhibitors of protein synthesis or nucleic acid synthesis. In other words, concentration-dependent antibiotics tend to have larger PAEs (14). For drugs with concentration-dependent bactericidal action the rate of bactericidal activity is maximal at the $\mathrm{C}_{\max }$ and higher doses of the drug not only increase the rate of reduction of bacteria, but also the length of time of drug exposure to bactericidal concentrations. Therefore the higher the drug concentration, the longer is the duration of the PAE for these drugs, and the less is the residual bacterial population at the time of the next dose (110). Hence from a PK/PD standpoint, large doses can be administered at longer dosing intervals because prolonged PAE protects against the bacterial re-growth when concentrations fall below the MIC.

Bacteria exposed to sub-MIC concentrations of antibiotics may undergo adaptive changes with modifications in cell wall structure, changes in ribosome density, or formation of filaments. In addition to morphological changes, sub-MICs may also have a direct inhibitory effect on the bacterial growth in vitro (111). Postantibiotic sub-MIC 
effect (PA SME) is another useful parameter which measures such effects of sub-MIC drug concentrations on bacterial growth following serial exposure to drug concentrations exceeding the MIC. This can be calculated as PA SME $=\mathrm{T}_{\mathrm{pa}}-\mathrm{C}$, where $\mathrm{T}_{\mathrm{pa}}$ is the time taken for the cultures previously exposed to antibiotics and then exposed to a sub-MIC to increase by one $\log _{10}$ above the counts observed immediately after washing/dilution and $\mathrm{C}$ is the corresponding time for the unexposed cultures (108).

Ginsburg et al. reported PAE properties of fluoroquinolones against MTB using an in vitro PK/PD model (112). By simulating PK parameters in humans, they determined the PAE of a single 400-mg dose of MXF on MTB to be over 1 week compared to a PAE of greater than 15 days in a traditional static in vitro study of MXF after an exposure period of $24 \mathrm{~h}$. Thus, such models can be effectively used to evaluate the post antibiotic effect of existing antimycobacterial agents by simulating a particular dosing regimen in vitro and studying the growth and kill rates after cessation of that specific therapy.

\section{Evaluation of Combination Therapy}

Simultaneous administration of antibiotics is always practiced in antituberculosis therapy because of the high potential for the development of TB drug resistance using monotherapy and the differing drug sensitivities of active and latent populations of the TB bacilli found within an established infection. There has also been substantial interest in novel combinations of new compounds with existing drugs to block the emergence of drug resistance and to shorten the duration of therapy. Testing new drugs or drug combinations for activity against tuberculosis in vivo is a highly expensive and timeconsuming process in animal models, and is largely prohibitive in humans for ethical reasons. In vitro models provide an inexpensive and rapid alternative for optimizing drug combinations before they proceed to preclinical or clinical testing. They also allow a high degree of standardization to study drug-receptor interactions without other interfering biological processes.

There are several existing methods to study antibiotic combinations in vitro. These include checkerboard techniques with tube dilutions or microtiter apparatus, the agar dilution method and various forms of disc proximity tests and paper strip methods (12, 113-115). However, all these methods involve constant concentrations of antibiotics to evaluate their activity.

When studying the combination of drugs and their time-kill curves in in vitro PK/PD models, differences in their pharmacokinetic properties can be taken into account. One of the major setbacks in this approach, however, is the complexity involved in effectively simulating concentration-time profiles of drugs with different half-lives. In spite of these intricacies, in vitro $\mathrm{PK} / \mathrm{PD}$ models for simultaneous simulation of serum kinetics of two or more drugs with different half-lives have been developed against fast growing organisms such as Streptococcus pneumonia and Staphylococcus aureus (96, 116-119). Although no examples have yet been reported for mycobacteria, similar 
models could likely be used to evaluate the synergistic potential of novel combinations of antituberculosis agents.

\section{Resistance}

Emerging resistance threatens the usefulness of available treatment options against the multi drug resistant mycobacteria. For example, MDR-TB takes about 18-24 months of therapy for complete cure as compared to a six month therapy against a susceptible strain. This prolonged duration of therapy is oftentimes also associated with severe toxicity related to the applied second line agents leading to poor patient compliance. Thus, it is desirable to develop novel drugs or drug combinations that are safe and effective against MDR strains. In vitro $\mathrm{PK} / \mathrm{PD}$ models can be effectively used to study such resistant clinical isolates for developing new molecules or new regimens with existing drugs. Conditions that can lead to resistant strains can be simulated and such resistant strains can further be studied.

Tam et al. used a hollow fiber model to examine the relationship between garenoxacin (a des-fluoroquinolone) and the likelihood to select resistant strains with different and escalating free area under the concentration-time curve over $24 \mathrm{~h}$ $\left(\mathrm{AUC}_{24}\right) / \mathrm{MIC}$ exposures of the drug for $48 \mathrm{~h}$ (120). The authors suggested that low $\mathrm{AUC}_{24} / \mathrm{MIC}$ ratios between 10 to 35 were optimal for maximally amplifying the preexisting resistant subpopulation. Exposures in excess of these ratios amplified the resistant subpopulation sub optimally, until an exposure was achieved that kept the number of resistant clones at or below the number present at the initiation of the therapeutic pressure resulting in an "inverted-U" shape for the function linking drug exposure to suppression of the resistant subpopulation.

Mutant prevention concentration (MPC) defines the antimicrobial drug concentration threshold that would require an organism to simultaneously possess two resistance mutations for growth in the presence of the drug. For M. bovis BCG, the MPC is estimated by determining the minimal antibiotic concentration that results in recovery of no mutants when large numbers of cells are applied to antibiotic-containing agar plates. The use of large numbers of cells, on the order of $10^{10}$ ensures that the restrictive antibiotic concentration blocks the growth of first-step mutants (121).

In one of the studies, the association between MPC based $\mathrm{PK} / \mathrm{PD}$ parameters (AUC/MPC, $\mathrm{C}_{\max } / \mathrm{MPC}$ or $\mathrm{T}>\mathrm{MPC}$ ) and emergence of resistant mutants of Streptococcus pneumoniae was evaluated for fluoroquinolones using an in vitro $\mathrm{PK} / \mathrm{PD}$ model. The results from this study identified $\mathrm{AUC}_{0-24} / \mathrm{MPC}$ and $\mathrm{C}_{\max } / \mathrm{MPC}$ indices to be associated with emergence of resistance (122). Although no such studies have been performed using mutants of MTB for emergence of resistance against antimycobacterials, it is plausible to evaluate these scenarios using in vitro $\mathrm{PK} / \mathrm{PD}$ models.

Population analysis profiles (PAP) is a specialized technique initially developed to detect vancomycin resistance but it can be applied to study drug resistant MTB strains. 
This technique compares the AUC of viable counts versus concentration profile of a strain under study against a control resistant strain after a given period of incubation. Based on this ratio one can determine if the test strain is a resistant one or not.

For example, in case of vancomycin resistant Staphylococcus aureus (VRSA), a ratio of AUC of a test strain against AUC of a hetero-resistant control strain Mu3 is calculated. This ratio exceeding 0.9 is used as a criterion to establish a vancomycin hetero-resistant $S$. aureus (hVRSA) $(123,124)$. In vitro PK/PD models can be used to perform such analyses. They can also be used to study the population dynamics of the susceptible and resistant strains over time following a particular dosage regimen (81). Although no such reports have been published so far for antituberculosis drugs, similar studies can be performed using MDR TB and XDR TB in order to establish effective dosing regimens against drug resistant strains.

\section{Pharmacokinetic/Pharmacodynamic Data Analysis}

PK/PD modeling and simulation techniques are increasingly used in drug development in a variety of indications. Such techniques allow characterizing the time course of the effect intensity resulting from a certain dosing regimen (2) and have been widely applied to antibiotics for evaluating their pharmacodynamics (their initial rate of the bacterial killing and the re-growth of bacteria) and to obtain a relevant PK/PD index which correlates with a therapeutic outcome.

Mathematical modeling of drug effects maximizes the information gained from an experiment, provides further insight into the mechanisms of drug effects and allows for simulations in order to design studies or even derive clinical treatment strategies (125). Modeling approaches can be classified into mechanism-based or empirical.

\section{Mechanism Based Modeling for Estimating PK/PD Parameters}

A mechanistic model is by definition a mathematical model based on known or hypothesized mechanisms of behavior of a biological system. The parameters are in accordance with pharmacokinetic, physicochemical, biophysical, physiological and patho-physiological principles, and have direct identifiable biological or biophysical interpretation (126). Mechanism based models for antimicrobials utilize drug concentrations and relate them to their effect. They are commonly applied to estimate the PK/PD parameters of antibiotic drugs and have to include at least a submodel of microorganism replication, a submodel of antimicrobial drug effects and a submodel for changing drug concentrations (pharmacokinetic submodel) (125). Since these models are limited to in vitro data, a host defense submodel is usually not included.

The submodel for microorganism replication is most commonly modeled using the following equation: 


$$
\frac{d N}{d t}=N \cdot k_{\text {replication }}-N \cdot k_{\text {death }}
$$

where $\mathrm{N}$ is the number of microorganisms and the first-order rate constants $\mathrm{k}_{\text {replication }}$ and $\mathrm{k}_{\text {death }}$ describe natural replication and death of microorganisms in the absence of antibacterial agents. This model, however, is based on the underlying assumption that the number of microorganisms $\mathrm{N}$ that can replicate is the same as the number subjected to death. Also this model cannot adequately describe the decrease in the net growth rate as the system approaches the stationary phase of bacterial growth. $k_{\text {growth }}$ or $k_{\text {apparent }}$, a first order rate constant for observed growth, which is the difference between $\mathrm{k}_{\text {replication }}$ and $\mathrm{k}_{\text {death }}$ is often applied because of difficulties in separating microorganism replication and death by a traditional kill curve analysis (125).

Increased knowledge regarding the production and the nature of persister cells, i.e., cells with reduced growth rates and reduced antibiotic susceptibilities can be efficiently applied to build mechanism-based PK/PD models (127). Nielsen et al. described a semi-mechanistic PK/PD model for assessment of antibacterial agents where the total bacterial population was divided into growing $(\mathrm{S})$ and resting $(\mathrm{R})$ population allowing a transfer from the growing population to the resting population as the total population approaches the stationary phase. Mathematically this can be modeled using the equations below:

$$
\begin{gathered}
\frac{d S}{d t}=S \cdot k_{\text {growth }}-S \cdot k_{\text {death }}-S \cdot k_{S R} \\
\frac{d R}{d t}=S \cdot k_{S R}-R \cdot k_{\text {death }}
\end{gathered}
$$

where $\mathrm{k}_{\mathrm{SR}}$ is the transfer rate constant (128).

To incorporate the antimicrobial effect, a submodel of antimicrobial drug effect relating the drug concentrations to the microbial survival is often described using the following equation:

$$
\frac{d N}{d t}=E_{\text {replication }} \cdot N \cdot k_{\text {replication }}-E_{\text {death }} \cdot N \cdot k_{\text {death }}
$$

The $\mathrm{E}_{\max }$ model can be used to describe between drug concentration and its effect on replication and death rate of the bacteria. The maximum effect obtained when determining a concentration-effect relationship $\left(\mathrm{E}_{\max }\right)$ and the concentration required to produce half-maximal effect $\left(\mathrm{EC}_{50}\right)$ are $\mathrm{PD}$ parameters used in defining the $\mathrm{E}_{\max }$ model. The $\mathrm{E}_{\max }$ model can also be used to describe the relationship between a PK/PD index and the effect. Drug effect decreasing the replication rate $\left(\mathrm{E}_{\text {replication }}\right)$ can be modeled using an inhibitory sigmoid $\mathrm{E}_{\max }$ model shown below:

$$
E_{\text {replication }}=1-\frac{I_{\max } \cdot C^{\gamma}}{I C_{50}^{\gamma}+C^{\gamma}}
$$


where $I_{\max }$ is the maximum inhibitory effect, $C$ is the drug concentration, $\gamma$ is the Hill coefficient and $\mathrm{IC}_{50}$ is the concentration that produces half-maximal inhibition.

Drug effect leading to increased death rate $\left(\mathrm{E}_{\text {death }}\right)$ can be modeled using a stimulatory sigmoid $\mathrm{E}_{\max }$ model as follows:

$$
E_{\text {death }}=1+\frac{E_{\max } \cdot C^{\gamma}}{E C_{50}^{\gamma}+C^{\gamma}}
$$

where $E_{\max }$ is the maximum stimulatory effect, $C$ is the drug concentration, $\gamma$ is the Hill coefficient and $\mathrm{EC}_{50}$ is the concentration that produces half-maximal stimulation leading to death.

It is well known that bacteria show different growth phases and that antibioticinduced killing often shows an initial phase with rapid killing, followed by a decline in the killing rate with time. Therefore ignoring the replication inhibition and including stimulation of death rate in Eq. 1-4 leads to the equation shown below:

$$
\frac{d N}{d t}=N \cdot k_{\text {replication }}-N \cdot k_{\text {death }}\left(1+\frac{E_{\max } \cdot C^{\gamma}}{E C_{50}^{\gamma}+C^{\gamma}}\right)
$$

with observed growth $\left(k_{\text {growth }}=k_{\text {replication }}-k_{\text {death }}\right)$ and a maximum kill rate $\left(k_{\text {kill }}\right.$ or $\left.k_{\max }=k_{\text {death }} \cdot E_{\max }\right)$, this equation can be further transformed into the most frequently applied PK/PD model for antibacterial drugs (125):

$$
\frac{d N}{d t}=N \cdot k_{\text {growth }}-N \cdot k_{\max } \cdot \frac{C^{\gamma}}{E C_{50}^{\gamma}+C^{\gamma}}
$$

The pharmacokinetic submodel describes the relationship between dose or dosing regimen and drug concentrations with time. Depending on the in vitro setup, this can be modeled as a one compartment model or a multi-compartment model. Assuming dosing by an intravenous bolus administration, a general form of the model can be defined as:

$$
C_{t}=A_{1} e^{-\alpha_{1} t}+A_{2} e^{-\alpha_{2} t}+\ldots .+A_{n} e^{-\alpha_{n} t}
$$

where $A_{1}$ to $A_{n}$ are coefficients, $\alpha_{1}$ to $\alpha_{n}$ are first order rate constants and $n$ denotes the number of compartments. This submodel can also be applied to approximate the concentration-time profiles for orally administered drugs that are rapidly absorbed.

Budha et al. reported the relationship between INH exposure and mycobacterial kill using a modified version of Eq. 1-8 as shown below, which was initially developed for Voriconazole against Candida isolates $(19,129)$ :

$$
\frac{d N}{d t}=\left[k_{0} \cdot\left(1-\frac{N}{N_{\max }}\right)-\left(1-e^{-\alpha \cdot t}\right) \cdot\left(\frac{I_{\max } \cdot C}{I C_{50}+C}\right)\right] \cdot N
$$


where $\mathrm{N}$ is the mycobacterial cell counts in $\mathrm{CFU} / \mathrm{mL}, \mathrm{k}_{0}$ is the bacterial net growth rate constant, $\mathrm{N}_{\max }$ is the maximum number of bacteria in the system in $\mathrm{CFU} / \mathrm{mL}, \mathrm{I}_{\max }$ is the maximum kill rate, $\mathrm{C}$ is the concentration of $\mathrm{INH}$ at time $\mathrm{t}$, and $\mathrm{IC}_{50}$ is the concentration at half-maximal kill rate. A logistic growth function, 1-N/N $\mathrm{N}_{\max }$ was used to describe the limited growth of bacteria in the absence of INH.

The authors reported a delay in bacterial kill which can likely be due to the time necessary to achieve sufficient intracellular drug exposure to initiate the killing process that was modeled using the term $1-e^{-\alpha \cdot t}$, where $\alpha$ is the delay rate constant.

When studying pharmacodynamics of antibiotics against MTB, an adaptive $\mathrm{IC}_{50}$ is often used $(19,102,129)$. This model component is necessary to explain the change in $\mathrm{IC}_{50}$ over the course of treatment due to an increase in drug insensitive cell population, for example due to latency for Mycobacterium species. The adaptive $\mathrm{IC}_{50}$ can be modeled using the equation below:

$$
I C_{50, A}=I C_{50} \cdot e^{\left(\frac{k_{\text {adaptation }} \cdot N_{0}}{N \cdot A U C_{0-24}}\right) \cdot t}
$$

where $\mathrm{IC}_{50, \mathrm{~A}}$ is the adaptive $\mathrm{IC}_{50}, \mathrm{~N}_{0}$ is the number of bacterial cells at time zero, $\mathrm{N}$ is the cell count in $\mathrm{CFU} / \mathrm{mL}$, $\mathrm{k}_{\text {adaptation }}$ is the adaptation constant and $\mathrm{IC}_{50}$ is the baseline $\mathrm{IC}_{50}$.

Gumbo et al. reported a mechanistic PK/PD model for MXF (99) which has been further applied in modeling INH (101) and RIF (103) PK/PD data. The resultant changes in the drug-resistant [R] and the drug-sensitive [S] Mycobacterium tuberculosis population were described using the equations below:

$$
\begin{array}{ll}
\frac{d N_{S}}{d t}=k_{\text {gmax }-S} \cdot\left(1-L_{S}\right) \cdot N_{S} \cdot E-k_{\text {kmax }-S} \cdot M_{S} \cdot N_{S} & \text { Eq. 1-12 } \\
\frac{d N_{R}}{d t}=k_{\text {gmax }-R} \cdot\left(1-L_{R}\right) \cdot N_{R} \cdot E-k_{k \max -R} \cdot M_{R} \cdot N_{R} & \text { Eq. 1-13 }
\end{array}
$$

where each subpopulation has an independent growth rate constant (drug sensitive, $\mathrm{K}_{\text {gmax- }}$ $\mathrm{s}$, drug resistant, $\mathrm{K}_{\mathrm{gmax}-\mathrm{R}}$ ). Bacteria at the site of infection which were in the logarithmic growth phase in the absence of drug exhibited an exponential density-limited growth rate described by the following equation:

$$
E=1-\left(N_{R}+N_{S}\right) / P O P M A X
$$

where $\mathrm{E}$ is the logistic growth term, and the maximal bacterial density (POPMAX) is identified as part of the estimation process. The authors allowed drug to affect the growth rate independently of kill through a saturable Michaelis-Menten type kinetic event (L), and the killing effect of the drug was modeled as a saturable Michaelis-Menten type kinetic event $(\mathrm{M})$ that relates the kill rate to drug concentration, where $\mathrm{C}_{50-\mathrm{g}}$ and $\mathrm{C}_{50-\mathrm{k}}$ is the drug concentration at which the bacterial growth or kill rate is half-maximal as described in the following equations: 


$$
\begin{aligned}
& L=\left(\frac{X_{1}}{V_{C}}\right)^{H} /\left[\left(\frac{X_{1}}{V_{C}}\right)^{H}+C_{50-g}{ }^{H}\right] \\
& M=\left(\frac{X_{2}}{V_{C}}\right)^{H} /\left[\left(\frac{X_{2}}{V_{C}}\right)^{H}+C_{50-k}{ }^{H}\right]
\end{aligned}
$$

where $\mathrm{H}$ is the slope constant, $\mathrm{X}_{1}$ and $\mathrm{X}_{2}$ are the amounts of drug in the central and the peripheral compartments of the hollow fiber system, respectively, and $\mathrm{V}_{\mathrm{c}}$ is the volume of the central compartment of the hollow fiber system. For drug-sensitive and drug-resistant populations as expressed in Eq. 1-13 and Eq. 1-14, there are separate terms for $\mathrm{H}, \mathrm{C}_{50-\mathrm{g}}$ and $\mathrm{C}_{50-\mathrm{k}}$.

\section{Empirical Modeling for Identifying Appropriate PK/PD Indices}

An empirical model is a non-mechanistic model whose parameters may be adequate for describing the longitudinal data at hand but does not take to underlying biological mechanisms into account. It can be predictive when used in conditions sufficiently similar to the ones already studied, but has usually limited predictability beyond these conditions (126). A typical empirical modeling exercise utilizes a timeintegrated measures of exposure, such as AUC or dose, from in vitro time-kill experiments to determine the primary PK/PD index responsible for the antibacterial effect and to understand whether a molecule exhibits a time-dependent or an exposuredependent killing pattern.

AUC/MIC, $\mathrm{C}_{\max } / \mathrm{MIC}$ and $\mathrm{T}>\mathrm{MIC}$, as discussed in the previous sections, are the three most commonly used PK/PD indices to evaluate antibacterial effects. Using a combination of dose escalation, fractionation and different strains one can identify the best suitable index related to the antibacterial effect for a specific compound (81). A sigmoid inhibitory dose-response model is one of the most frequently used models to characterize antimicrobial activity. For instance, in one of the recent studies for identification of the most appropriate empirical PK/PD index associated with the microbial kill of the first line antituberculosis agent INH, viable cell counts on different days following multiple dosing of INH were analyzed using an inhibitory $\mathrm{E}_{\max }$ model shown below:

$$
E=E_{\text {control }}-\left(\frac{E_{\text {max }} \cdot P K P D}{E C_{50}+P K P D}\right)
$$

where $\mathrm{E}$ is the observed M. bovis $\mathrm{BCG}$ cell counts in $\log _{10} \mathrm{CFU} / \mathrm{mL}, \mathrm{E}_{\text {control }}$ is the cell count in the control experiment, and $\mathrm{E}_{\max }$ is the maximal antimicrobial effect in $\log _{10} \mathrm{CFU} / \mathrm{mL} . \mathrm{EC}_{50}$ is the value of the $\mathrm{PK} / \mathrm{PD}$ index that produces half-maximal antimicrobial effect and PKPD is one of the empirical PK/PD indices $\mathrm{AUC}_{0-24} / \mathrm{MIC}$, $\mathrm{T}>\mathrm{MIC}$ or $\mathrm{C}_{\max } / \mathrm{MIC}$. INH exhibited exposure-dependent antibacterial activity on $M$. bovis $\mathrm{BCG}$ where the empirical $\mathrm{PK} / \mathrm{PD}$ index $\mathrm{AUC}_{0-24} / \mathrm{MIC}$ was found to be well associated with the microbial kill (19). 
For the first line antituberculosis agents INH, RIF and Pyrazinamide (PZA) and second line fluoroquinolones and aminoglycosides, a concentration dependent killing pattern that correlates best with AUC/MIC ratio is shown to be a reliable predictor of efficacy $(19,99-104)$.

\section{Drawbacks of In Vitro Models}

In spite of the potential advantages of in vitro models discussed in this manuscript, these models are obvious simplifications of in vivo scenarios and therefore come with certain drawbacks which cannot be ignored. These can be classified under host factors, pathogen factors and pharmacokinetic factors.

\section{Host Factors}

The host immune system plays a major role in TB. Most infected immunocompetent individuals fail to progress to full-blown disease because the MTB bacilli are directly killed by macrophages or walled off by the immune system inside a tissue nodule known as a granuloma. The granuloma's primary function is to contain and prevent the dissemination of the mycobacteria (130). Human tuberculous granulomas contain an organized collection of differentiated and activated macrophages, $\mathrm{T}$ lymphocytes, some B-lymphocytes, dendritic cells, neutrophils, fibroblasts and extracellular matrix components that limit nutrient delivery to the core (131). Bacteria are often found multiplying inside macrophages around the edge of the necrotic center of a granuloma. In vitro PK/PD models lack the ability to mimic this complex host defense mechanism and therefore approximate more to conditions of an immunodeficient patient where the infection is more bacteremic in nature (92). However, during the reactivation phase of the disease the majority of the bacilli in pulmonary cavities reside extracellularly (132), and hence the results obtained from in vitro models may be reflective of the in vivo killing under this condition.

Besides the lack of immune response, physiological conditions also vary between in vivo and in vitro setups leading to differences in antituberculosis activity. For example the front line antituberculosis drug pyrazinamide is inactive in normal culture conditions and is only active at acidic pHs that are thought to mimic those experienced by bacteria in activated macrophages. The tuberculosis granuloma is also believed to be subjected to a gradient of microaerophilic/ anaerobic conditions which limits growth. Since these conditions are technically difficult to simulate in vitro, antibacterial efficacy can differ significantly between the in vitro model and the in vivo environment.

\section{Pathogen Factors}

The growth rate for most bacteria is faster in vitro compared to growth in vivo or in human serum. Since antimicrobial efficacy in an in vitro model relates to the rate of 
bacterial growth, this can be a potential limitation of in vitro models, especially when there are significant differences in the growth rates $(133,134)$. Biochemical data suggest that MTB growing in vivo upon granuloma formation shifts to anaerobic metabolic pathways. It has also been shown that tubercle bacilli are able to shift into a nonreplicating drug tolerant state when nutrients are depleted. This process gives MTB the capability to lie dormant in the host for long periods of time and this results in differences in growth conditions in vitro and in vivo (23).

Furthermore, the growth rate of MTB within the infected host varies according to the type of lesion such as extracellular and actively multiplying bacilli in the liquefied caseous material covering the cavity wall, semi-dormant bacilli with intermittent bursts of metabolic activity inhabiting solid caseous material and a small population of bacilli within the acidic environment around the areas of active inflammation or necrosis. The growth characteristics within each lesion result in differing susceptibility to specific antituberculosis agents (25) and simulating such varying populations in vitro is very challenging. Only a few studies have been reported so far where different metabolic populations of MTB growing under acidic conditions and under anaeroboic/hypoxic conditions have been subjected to antituberculosis drug treatment using in vitro $\mathrm{PK} / \mathrm{PD}$ models (104).

\section{Pharmacokinetic Factors}

Drug distribution is technically challenging to simulate in vitro. Using complex in vitro setups, one can approximate the in vivo elimination process of the drug, but the model may not truly represent the in vivo drug distribution. Plasma protein binding of the antibiotic is absent in in vitro models and needs to be accounted for. Thus, free drug concentrations relevant to the site of infection must be simulated. Although this is feasible, protein binding in vivo is a dynamic process and can be altered due to coexisting disease conditions or the presence of other drugs which is challenging to simulate in vitro.

\section{Summary}

In vitro experiments are useful in evaluating the direct interaction between antituberculosis agents and Mycobacterium tuberculosis, which aids in the selection of candidate compounds during antituberculosis drug development. Utilizing in vivo drug clearance profiles from animal and/or human studies and simulating them in an in vitro $\mathrm{PK} / \mathrm{PD}$ model allows the in depth characterization of antimycobacterial activity of new and existing anti-TB agents by generating time-kill data. These data capture the dynamic interplay between mycobacterial growth and changing drug concentration as encountered during prolonged drug therapy.

Pharmacokinetic parameters such as protein binding and drug distribution play an important role when extrapolating results from an in vitro experiment to humans or 
animals. While MIC and $\mathrm{MBC}$ are most commonly used in vitro pharmacodynamic parameters, $\mathrm{AUC} / \mathrm{MIC}, \mathrm{C}_{\max } / \mathrm{MIC}$ and $\mathrm{T}>\mathrm{MIC}$ are the three most commonly used $\mathrm{PK} / \mathrm{PD}$ indices to evaluate antimycobacterial effects of antituberculosis agents.

On the basis of drug exposure and bacterial concentration in vitro PK/PD models for antituberculosis agents can be classified into static models and dynamic models. While static models evaluate how a bacterial culture responds to a constant environment with a fixed antibiotic exposure, dynamic models utilize time-kill curves, which follow the mycobacterial killing and growth as a function of both time as well as antibiotic concentration. In vitro models can serve as useful tools to study postantibiotic effect, the emergence of resistance and help identify novel drug combinations to treat drug resistant mycobacterial strains.

$\mathrm{PK} / \mathrm{PD}$ modeling and simulation techniques allow characterizing the time course of the effect intensity of an antibiotic resulting from a certain dosing regimen and have been applied with success to antituberculosis agents for evaluating their initial rate of the bacterial killing and the re-growth of mycobacteria and to obtain a relevant PK/PD index which correlates with therapeutic outcome.

In spite of the potential advantages, in vitro models are obvious simplifications of in vivo scenarios and therefore come with certain drawbacks such as absence of immune response, divergent physiological conditions in vivo and differences in growth conditions of mycobacteria in vitro versus in vivo. Since these in vitro models have benefits of reduced cost, flexibility and adaptability, they are often used to guide the early drug development process, to minimize lengthy experiments for testing antituberculosis activity in vivo, and may help clinicians and researchers combat and treat tuberculosis patients, producing ultimately improved treatment outcomes. 


\section{CHAPTER 2. RESEARCH HYPOTHESIS}

Lead optimization is a complex process of refining the chemical structure of a screen lead to improve its drug-like properties in order to successfully produce a development candidate. Using an iterative approach the knowledge gained at each stage is used to further optimize each new cycle. In an early development phase, this tends to be chemistry intensive and the optimization is carried out after evaluating the pharmacological, biopharmaceutic and pharmacokinetic profile using a variety of in vitro and in vivo methods. One of the tasks towards achieving this goal is to develop a comprehensive absorption, distribution, metabolism and elimination (ADME) package which includes data on absorption/permeability, mechanisms of clearance, oral bioavailability, interaction with CYP enzymes to evaluate metabolic stability, and plasma protein binding (135). For an antituberculosis drug in particular, the effectiveness depends upon its ability to reach the site of infection in a sufficiently high unbound concentration and to remain at the site of infection for a sufficiently long period of time. Along with that, the compound should possess the ability to penetrate the bacterial cell wall to achieve sufficient intracellular concentrations. In order to target the primary phase of TB infection, the drug should also be able to penetrate into macrophages. Once it reaches its target, the compound should have good intrinsic activity towards its target to achieve the required antimicrobial effect.

I hypothesized that designing a screening paradigm involving ADME and drug uptake assays into MTB as well as macrophages would facilitate the identification and characterization of lead compounds specific for antituberculosis therapy. To address this hypothesis, I utilized a series of existing in vivo and in vitro assays (Chapter 3 ) such as plasma protein binding, microsomal metabolic stability and in vivo pharmacokinetics, and developed novel drug uptake assays into M. bovis BCG and J774 murine macrophage to identify and characterize lead molecules from a novel spectinamide series of antituberculosis agents.

PK/PD concepts have recently been applied more widely in the drug development, lending statistical and causal support to the evaluation of the dose-response relationship and are advocated as a means of improving development efficiency (136). For rapid antituberculosis drug development in vitro PK/PD models are useful in evaluating the direct interaction between the drug and the bacteria, thereby guiding the selection of candidate compounds and the optimization of their dosing regimens. Utilizing in vivo drug-clearance profiles from animal studies and simulating them in an in vitro $\mathrm{PK} / \mathrm{PD}$ model allows the in-depth characterization of antibiotic activity of new and existing antibacterials by generating time-kill data. These data capture the dynamic interplay between mycobacterial growth and changing drug concentration as encountered during prolonged drug therapy in vivo (137).

Following the selection of a lead molecule, I hypothesized that simulating the in vivo pharmacokinetic profile at different dosing regimens in an in vitro $\mathrm{PK} / \mathrm{PD}$ system helps in characterizing the dose-response relationship which further facilitates rational 
dose selection for the in vivo efficacy study in animal models infected with TB. To address this hypothesis, we determined the time course of bacterial kill rates for dosing regimens of a spectinamide lead compound, Lee1445 in the in vitro PK/PD model (Chapter 4). Based on this data, we developed an in silico pharmacodynamic model for describing the Lee1445 mediated bacterial kill and applied this in silico model to predict the bactericidal effect of Lee1445 at different, untested dosing regimens by numerical simulation experiments. Based on the predictions, we suggested an optimal dosing regimen that should be tested in the in vivo efficacy study in order to maximize the efficacy in terms of reduction of mycobacterial load in the animal. 


\section{CHAPTER 3. PHARMACOKINETIC EVALUATION OF SPECTINAMIDES}

\section{Introduction}

TB is a contagious and airborne disease caused by Mycobacterium tuberculosis. According to the WHO global tuberculosis control report of 2010, approximately 1.7 million people died from TB in 2009 with a vast majority of deaths occurring in developing countries. There were 9.4 million new TB cases in 2009 of which 1.1 million cases were co-infected with HIV. The highest rates of MDR-TB so far have been reported in 2010, where patients failed to respond to the standard first-line drugs. About 58 countries reported XDR-TB which is resistance to the second-line agents on top of MDR-TB (1).

In the past decade there has been a reawakening of TB drug research and development was spurred by an urgent need to prevent the rising cases of this disease globally and develop new and more effective treatments against both drug sensitive and resistant strains. The primary goals are to shorten and simplify the treatment of active TB, provide safer and more efficacious treatments for drug-resistant TB, simplify treatment of TB-HIV co-infections by eliminating unwanted drug-drug interactions, and shorten treatment for latent TB infection (138).

From a biopharmaceutic and pharmacokinetic standpoint, an ideal TB drug should possess high oral bioavailability, since oral administration is the route of choice for better patient compliance, indicating that the compound should have good aqueous solubility and gastrointestinal permeability. In order to allow lesser frequency of dosing, it should possess a longer elimination half-life (5). To target the primary phase of TB, the drug should be able to penetrate into macrophages and also into the bacterial envelope assuming the drug target is located intracellularly. In an effort to develop more potent drugs for the treatment of TB, a novel class of antituberculosis agents called spectinamides, with promising in vitro activity against MTB, has been recently characterized by our group.

Spectinamides are novel amide derivatives of the antibiotic spectinomycin and have emerged as a new class of agents to treat tuberculosis. These agents showed potent in vitro activity against Mycobacterium tuberculosis compared to spectinomycin. In a preliminary in vivo study in IFN- $\gamma$ knockout mice, spectinamide Lee1329 reduced the lung bacillary load of TB comparable to streptomycin. The main objective of the work summarized in this chapter is to characterize the biopharmaceutic and pharmacokinetic properties of selected spectinamides and develop a rational screening paradigm which will further improve the early drug development process. This will enable us in iteratively optimizing these molecules via structural modification to further improve their PK properties. 


\section{Materials and Methods}

\section{Chemicals and Reagents}

All tested spectinamides were synthesized in the laboratory of Dr. R.E. Lee at St. Jude Children's Research Hospital, Memphis, TN, including Lee1329 (3'-dihydro-3'deoxy-4(R)-(pyridin-2-yl) acetylamino spectinomycin), Lee1443 (3'-dihydro-3'-deoxy4(R)-(thiazol-4-yl) acetylamino spectinomycin), Lee1445 (3'-dihydro-3'-deoxy-4(R)-(5fluoropyridin-2-yl) acetylamino spectinomycin), Lee1465 (3'-dihydro-3'-deoxy-4(R)(benzooxazol-2-yl) acetylamino spectinomycin), Lee1540 (3'-Dihydro-3'-deoxy-4(R)(2-((4-(trifluoromethoxy) phenyl) amino) thiazol-4-yl) acetylamino spectinomycin), and Lee1544 (3'-Dihydro-3'-deoxy-4(R)-(4-chloropyridin-2-yl) acetylamino spectinomycin) (Figure 3-1). Acetonitrile, Methanol, HPLC grade water, formic acid and ammonium formate were purchased from Fisher Scientific (Pittsburgh, PA). Drug free rat plasma was purchased from Aleken Biologicals (Nash, TX).

\section{Caco-2 Permeability Assay}

Caco-2 permeability data was obtained from the high throughput screening laboratory in the department of Chemical Biology and Therapeutics, St. Jude Children's Hospital, Memphis, TN. This assay was performed in the 96-well Transwell system. Caco-2 cells were maintained at $37^{\circ} \mathrm{C}$ in a humidified incubator with an atmosphere of $5 \% \mathrm{CO}_{2}$. The cells were cultured in minimum essential medium (MEM) containing 20\% fetal bovine serum (FBS) in $75 \mathrm{~cm}^{2}$ flasks, 100 units $/ \mathrm{ml}$ of penicillin, and $100 \mu \mathrm{g} / \mathrm{ml}$ of streptomycin. The Caco-2 cells were seeded onto inserts of a 96-well plate at a density of $0.165 \times 10^{5}$ cells/insert and cultured in the MEM containing 10\% FBS for 7 days. Each cultured monolayer on the 96-well plate was washed twice with HBSS/HEPES (10 mM, $\mathrm{pH}$ 7.4). The permeability assay was initiated by the addition of each compound solution $(50 \mu \mathrm{mol} / \mathrm{L})$ into inserts (apical side, $\mathrm{A})$ or receivers (basolateral side, B). The Caco-2 cell monolayers were incubated for $2 \mathrm{~h}$ at $37^{\circ} \mathrm{C}$. Fractions were collected from receivers (if apical to basal permeability) or inserts (if basal to apical permeability), and concentrations were assessed by UPLC/MS (Waters; Milford, MA). All compounds were tested in triplicates. The $\mathrm{A} \rightarrow \mathrm{B}$ ( or $\mathrm{B} \rightarrow \mathrm{A}$ ) apparent permeability coefficients (Papp, $\mathrm{cm} / \mathrm{s}$ ) of each compound were calculated using the equation, $\mathrm{Papp}=\mathrm{dQ} / \mathrm{dt} \times 1 / \mathrm{AC}_{0}$. The flux of a drug across the monolayer is $\mathrm{dQ} / \mathrm{dt}(\mu \mathrm{mol} / \mathrm{s})$. The initial drug concentration on the apical side is $\mathrm{C}_{0}(\mu \mathrm{mol} / \mathrm{L})$. The surface area of the monolayer is $\mathrm{A}\left(\mathrm{cm}^{2}\right)$.

\section{In Vitro Microsomal Metabolic Stability}

In vitro microsomal metabolic stability of the compounds was assessed using pooled liver microsomal preparations (Cellzdirect, Austin, TX). Reactions were started by adding $25 \mu \mathrm{L}$ of microsomal protein solution $(10 \mathrm{mg} / \mathrm{mL})$ to $25 \mu \mathrm{L}$ of test compound $(20 \mu \mathrm{M})$ and $200 \mu \mathrm{L}$ of NADPH regenerating solution $(1.3 \mathrm{mM} \mathrm{NADP}+, 3.3 \mathrm{mM}$ 


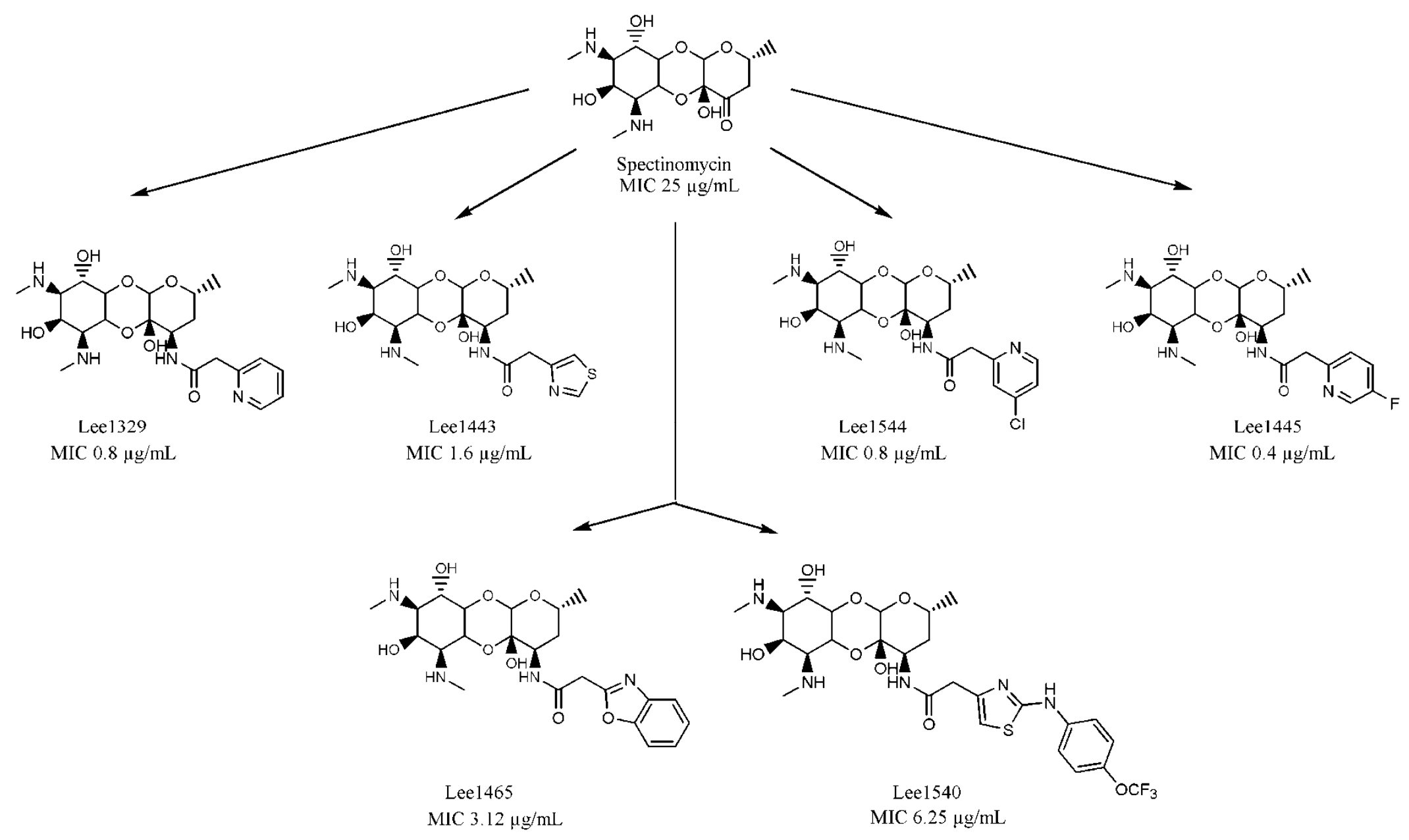

Figure 3-1. Chemical structures of spectinomycin, first generation 2-heteroaryl acetic acid amide analogs (Lee1329, Lee1443, Lee1445 and Lee1544), second generation fused ring analog (Lee1465) and second generation phenyl amino thiazole analog (Lee1540). 
glucose-6-phosphate, $3.3 \mathrm{mM} \mathrm{MgCl} 2$ and 1 unit/mL glucose-6-phosphate dehydrogenase in $\mathrm{pH} 7.4$ phosphate buffer solution). The reaction mixture was incubated at $37^{\circ} \mathrm{C}$ and samples were taken at $0,5,10,15,30,45,60$ and 90 minutes respectively. A reaction mixture containing above mentioned composition but instead using deactivated microsomes was used as negative control and a nitrofuranylamide with known significant phase I metabolism Lee1341 was used as positive control. All the samples were analyzed using LC-MS/MS assay. Disappearance of the parent compound was monitored during the incubation period. The percentage of parent compound remaining intact was estimated by comparing analyte concentrations before and after incubation.

\section{Protein Binding}

Protein binding of the compounds was determined using equilibrium dialysis. Biologically relevant concentrations of test compound were prepared (low and high) in plasma. $200 \mu \mathrm{L}$ of the plasma sample was placed in the central chamber and $350 \mu \mathrm{L}$ of blank isotonic phosphate buffer, $\mathrm{pH} 7.4$ was added to the peripheral chamber of a dialysis device (MW cutoff 6000-8000 D, RED ${ }^{\circledR}$ device, Pierce Biotechnology Inc, Rockford, IL). The chambers were covered with a seal and incubated at $37^{\circ} \mathrm{C}$ for four hours on a shaker set at $100 \mathrm{rpm}$. At the end of incubation, the volumes of plasma and recipient buffer were measured to identify and account for volume shift, if any. Aliquots of plasma and buffer were used to determine the drug concentration using an LC-MS/MS assay. The free fraction of the drug was calculated as ratio of the concentrations in the buffer and in plasma.

\section{Pharmacokinetic Studies}

Catheterized male Sprague-Dawley rats (jugular vein alone for oral study and jugular vein and femoral vein for intravenous study) weighing approximately $225 \mathrm{~g}$ were obtained from Harlan Bioscience (Indianapolis, IN). The animals were kept on a $12 \mathrm{hr}$ light/dark cycle with food and water available ad libitum. Groups of rats $(n=4)$ received either an intravenous (IV) or oral dose of a test compound at a dose level of $10 \mathrm{mg} / \mathrm{Kg}$ or $100 \mathrm{mg} / \mathrm{Kg}$, respectively. For oral administration, the animals were fasted overnight and until 4 hours after administration of test compound. Serial blood samples (approx. 250 $\mu \mathrm{L}$ ) were collected pre-dose and at predetermined time points post-dose until 48 hours. Plasma was separated immediately by centrifugation $\left(10,000 \mathrm{rpm}\right.$ for $10 \mathrm{~min}$ at $\left.4{ }^{\circ} \mathrm{C}\right)$ and stored at $-80^{\circ} \mathrm{C}$ until analysis. Urine specimens were collected for a period of 48 hours following drug administration. The study protocol was approved by the institutional animal care and use committee of the University of Tennessee Health Science Center.

\section{Sample Preparation and LC-MS/MS Assay}

A calibration curve ranging from 3.9-5000 $\mu \mathrm{g} / \mathrm{L}$ was constructed for each test compound by spiking the test compound into $50 \mu \mathrm{L}$ of blank rat plasma. A structurally 
similar analogue to the test compounds, Lee1369, was used as internal standard (IS) to all calibration standards and all plasma specimens. Plasma proteins were precipitated by the addition of four volumes of ice cold methanol containing IS. These samples were vortexed and kept on ice for 20 minutes. Following this, the samples were centrifuged at $10,000 \mathrm{rpm}$ for 10 minutes at $4{ }^{\circ} \mathrm{C}$ and the supernatants were diluted if necessary and injected into LC-MS/MS system for analysis. Chromatographic separations were carried out using a Shimadzu liquid chromatography system (Shimadzu Corporation, USA) consisting of two pumps, online degasser, system controller and a CTC Leap auto sampler (Leap Technologies, Carrboro, NC). A gradient of methanol and $10 \mathrm{mM}$ ammonium acetate at $\mathrm{pH} 3.5$ was used at a flow rate of $0.4 \mathrm{~mL} / \mathrm{min}$. A Phenomenex ${ }^{\circledR}$ Luna $3 \mu$ HILIC, $100 \times 4.6 \mathrm{~mm}$ column (Phenomenex, Torrance, CA) protected with a guard column was used for the separation. $10 \mu \mathrm{L}$ of sample was injected onto the column and the elute was led directly into an API 3000 triple-quadrupole mass spectrometer (Applied Biosystems ABI/MDS-Sciex, Foster City, CA) equipped with an electrospray ion source. The instrument was operated in the positive ion mode with nebulizer gas (NEB) at $7 \mathrm{psi}$, curtain gas (CUR) at 8 psi, collision gas (CAD) at 10 psi, ion spray voltage (IS) at $+4000 \mathrm{~V}$ and temperature (TEM) at $500{ }^{\circ} \mathrm{C}$. The resulting multiple reaction monitoring chromatograms were used for quantification using the Analyst software version 1.4.1 (Applied Biosystems ABI/MDS-Sciex, Foster City, CA).

\section{Pharmacokinetic Data Analysis}

Plasma concentration-time data obtained from oral and intravenous administration were analyzed by non-compartmental analysis. Also, a two compartment open model with bolus input and first order output was used to analyze the IV plasma concentrationtime data. The area under the plasma concentration-time curve from time zero to infinity $\left(\mathrm{AUC}_{\text {inf }}\right)$ was calculated by the trapezoidal rule with extrapolation to time infinity. Mean residence time (MRT), the average amount of time a particle remains in a compartment of system was calculated for IV dose using $\mathrm{MRT}=\mathrm{AUMC} \mathrm{C}_{\text {inf }} / \mathrm{AUC}_{\text {inf }}$ where $\mathrm{AUMC}_{\text {inf }}$ is the area under the moment curve when the concentration-time curve is extrapolated to infinity. The systemic clearance (CL) was calculated using the equation $\mathrm{CL}=$ Dose $_{\mathrm{iv}} / \mathrm{AUC}_{\mathrm{inf}, \mathrm{iv}}$, where Dose ${ }_{\mathrm{iv}}$ and $\mathrm{AUC}_{\mathrm{inf}}$, iv are the IV dose and corresponding area under the plasma concentration-time curve from time 0 to infinity, respectively. An estimate of volume of distribution at steady state $\left(\mathrm{V}_{\mathrm{ss}}\right)$ was obtained from IV data using $\mathrm{V}_{\mathrm{ss}}=\mathrm{MRT}^{*} \mathrm{CL}$. Oral bioavailability $(\mathrm{F})$ was calculated using $\mathrm{F}=\left(\mathrm{AUC}_{\mathrm{inf}}\right.$, oral $\left.* \mathrm{Dose}_{\mathrm{iv}}\right) /$ $\left(\mathrm{AUC}_{\text {inf, iv }}{ }^{*}\right.$ Dose $\left._{\text {oral }}\right)$, where Dose oral $_{\text {, Dose }}, \mathrm{AUC}_{\text {inf, iv }}$, and $\mathrm{AUC}_{\text {inf, oral }}$ are the oral and IV doses and the corresponding areas under the plasma concentration-time curves from time zero to infinity, respectively. Urinary excretion parameters were calculated from the cumulative dose excreted unchanged in urine and the fraction unbound in plasma $\left(f_{u p}\right)$. Glomerular filtration rate (GFR) and hepatic blood flow $\left(\mathrm{Q}_{\mathrm{h}}\right)$ were taken from the physiologic parameters published by Davies et al. (139). The fraction $\left(f_{e}\right)$ of the test compound excreted in urine was calculated as the cumulative amount of dose excreted unchanged in urine divided by the dose of the test compound administered. Renal clearance (CLr) values were determined from the total plasma CL and the fraction excreted in urine using $\mathrm{f}_{\mathrm{e}}{ }^{*} \mathrm{CL}_{\text {total }}$. The net process a drug undergoes in the kidney can be 
evaluated using excretion ratio ( $\left.\mathrm{E}_{\text {ratio }}\right)$. This was calculated using $\mathrm{CLr} /\left(\mathrm{f}_{\mathrm{up}}\right.$ *GFR). Nonrenal clearance $\left(\mathrm{CL}_{\mathrm{nr}}\right)$ was calculated using $\mathrm{CL}_{\text {total }}-\mathrm{CL}_{\mathrm{r}}$ and the hepatic extraction ratio was calculated using $\mathrm{CL}_{\mathrm{nr}} / \mathrm{Q}_{\mathrm{h}}$.

\section{Drug Uptake into M. bovis BCG}

To study the uptake of spectinamide series of anti-tuberculosis agents into $M$. bovis BCG, bacterial cultures were grown in Middlebrook $7 \mathrm{H} 9$ medium (Becton Dickinson, Sparks, MD) with 10\% albumin dextrose complex and $0.1 \% \mathrm{v} / \mathrm{v}$ Tween 80 to late $\log$ phase $\left(\mathrm{OD}_{600}=0.75\right)$. These cultures were concentrated following a series of centrifugation steps to obtain a final cell density of approximately $10^{11}$ cells $/ \mathrm{mL}$. Concentrated culture suspension $(1 \mathrm{~mL})$ was added to pre-weighed centrifuge tubes. To this suspension, concentrated drug stock was added to obtain a final drug concentration of $25 \mu \mathrm{g} / \mathrm{mL}$ and was incubated at $37^{\circ} \mathrm{C}$ for 4 hours. Following incubation, the suspension was centrifuged and the supernatant discarded. The cell pellet was washed thrice with DPBS (pH 7.0) and resuspended with $0.8 \mathrm{~mL}$ of sterile distilled water. This was bead beat using $0.1 \mathrm{~mm}$ glass beads three times (10 cycles of 10 seconds each time). The cell lysate was then sterile filtered and analyzed for drug content using a liquid chromatography-tandem mass spectrometric (LC-MS/MS) assay.

\section{Drug Uptake into Murine Macrophages}

To study the uptake of spectinamide antibiotics into murine macrophages, J774 cells were grown to confluence in Dulbecco's Modified Eagle's Medium with $10 \%$ fetal bovine serum (ATCC, Manassas, VA) in tissue culture flasks maintained at $37^{\circ} \mathrm{C}$ in $5 \%$ $\mathrm{CO}_{2}$ incubator. These adhered cells were then scraped using a teflon cell scraper attached to a rubber policeman following aspiration of old media and addition of fresh media. The cells were counted after they were stained with tryphan blue using a hemocytometer on inverted microscope. The cell density was adjusted to $4 \times 10^{5}$ cells $/ \mathrm{mL}$ using fresh media. The cell suspension was then added to new tissue culture flasks and was kept overnight to allow the cells to adhere. Media containing a final drug concentration of $25 \mu \mathrm{g} / \mathrm{mL}$ was added to these flasks following aspiration of old media and incubated for $24 \mathrm{~h}$ at $37{ }^{\circ} \mathrm{C}$ in a $5 \% \mathrm{CO}_{2}$ incubator. Following incubation the cells were washed thrice with DPBS $(\mathrm{pH}$ 7.0). These cells were then scraped and resuspended in $0.6 \mathrm{~mL}$ sterile distilled water and were subjected to lysis using a bead beater $(0.1 \mathrm{~mm}$ glass beads, three repeats of 10 cycles of 10 seconds each). The cell lysate was then sterile filtered and analyzed for drug content using a liquid chromatography-tandem mass spectrometric (LC-MS/MS) assay.

Cell cytotoxicity was studied for a range of drug concentrations $(1.56 \mu \mathrm{g} / \mathrm{mL}$ to $200 \mu \mathrm{g} / \mathrm{mL}$ ) using the MTT cell proliferation assay (ATCC, Manassas, VA). $100 \mu 1$ of each of these concentrations were added in triplicate in a 96 well plate and to each of these wells $100 \mu \mathrm{l}$ of media containing J774 cells was added so that each well approximately has $10^{3}$ to $10^{5}$ cells/well. A well containing cells without addition of drug was used as a control. These cells were incubated for 24 hours and $20 \mu 1$ of MTT reagent 
was added to each of these wells. These cells were incubated for 4 hours until a purple precipitate was visible. $100 \mu \mathrm{l}$ of detergent reagent to each of these wells and was left at room temperature in the dark for 2 hours. Following this, absorbance at $570 \mathrm{~nm}$ was recorded and the ratio of absorbance of drug treated cells to untreated control was calculated which was converted to percentage activity or cell viability.

\section{Results and Discussion}

\section{LC-MS/MS Assay Performance}

\section{Linearity and Calibration Standards}

The peak area ratios of analyte to IS were linear over a concentration range of 3.9$5000 \mu \mathrm{g} / \mathrm{L}$ for spectinamides. The best fit for the calibration curve was achieved by a linear equation of $\mathrm{y}=\mathrm{mx}+\mathrm{c}$ and a $1 / \mathrm{x}^{2}$ weighting factor with minimum of residuals and regression coefficients $>0.992$ to 0.998 .

\section{Specificity and Selectivity}

Chromatograms of control drug free plasma contained no co-eluting peaks of analyte area at the lowest limit of quantitation (LLOQ) level and no co-eluting peaks $>5 \%$ of the area of IS. There was no cross interference between the analytes. The retention times of analyte and IS showed low variability with a relative standard deviation (RSD) well within the acceptable limit of 5\%.

\section{Limit of Detection (LOD) and Lowest Limit of Quantitation (LOQ)}

The LOD for which the analyte gave a signal-to-noise ratio $(\mathrm{S} / \mathrm{N})$ of $\geq 3$ was determined as $1.95 \mu \mathrm{g} / \mathrm{L}$ concentration. The LLOQ, the lowest concentration in the standard curve which can be measured with acceptable accuracy and precision for spectinamides from normal rat plasma, was established as $3.9 \mu \mathrm{g} / \mathrm{L}$, with an exception of Lee1443 (LLOQ of $7.8 \mu \mathrm{g} / \mathrm{L}$ ).

\section{Biopharmaceutical Properties}

All active spectinamides have molecular weights below 500 Da except for Lee1540 (Table 3-1). While $\log$ P refers to the logarithm of the Partition Coefficient, P, which is defined as the ratio of concentration of neutral species in octanol divided by the concentration of neutral species in water, $\log \mathrm{D}$ refers to the logarithm of the distribution coefficient, $\mathrm{D}$, which is defined as the ratio of concentrations of all species i.e., neutral and ionized, present in octanol divided by the concentration of all species in water. In silico calculated $\log \mathrm{P}(\mathrm{CLog} \mathrm{P})$ values (CS ChemDraw, CambridgeSoft Corporation, MA) as well as calculated $\log \mathrm{D}$ values (MarvinView, ChemAxon Ltd., Hungary) suggested 
Table 3-1. Physicochemical and biopharmaceutical properties of spectinamides.

\begin{tabular}{|c|c|c|c|c|c|c|c|}
\hline \multirow[b]{2}{*}{ Molecule } & \multirow[b]{2}{*}{$\begin{array}{c}\mathbf{M W} \\
(\mathrm{Da})\end{array}$} & \multirow[b]{2}{*}{ CLogP } & \multirow[b]{2}{*}{$\begin{array}{c}\text { LogD } \\
\text { pH 7.0 }\end{array}$} & \multirow[b]{2}{*}{$\begin{array}{c}\text { tPSA } \\
\left(\AA^{2}\right)\end{array}$} & \multicolumn{3}{|c|}{ Caco-2 permeability } \\
\hline & & & & & $\begin{array}{c}\mathbf{P}_{\text {app }} \\
(\mathbf{A} / \mathbf{B}) \\
(\mathrm{nm} / \mathrm{s})\end{array}$ & $\begin{array}{c}\mathbf{P}_{\text {app }} \\
\text { (B/A) } \\
(\mathrm{nm} / \mathrm{s})\end{array}$ & $\begin{array}{c}\text { Efflux } \\
\text { ratio } \\
\text { B2A/A2B }\end{array}$ \\
\hline Spectinomycin & 332.3 & -2.87 & -3.93 & 129.5 & 224.5 & 176.1 & 0.78 \\
\hline Lee1329 & 452.5 & -3.31 & -4.14 & 153.9 & 46.4 & 76.6 & 1.65 \\
\hline Lee1443 & 458.5 & -3.47 & -4.26 & 153.9 & 89.6 & 115.0 & 1.28 \\
\hline Lee 1445 & 470.5 & -3.08 & -4.00 & 153.9 & 145.0 & 149.5 & 1.03 \\
\hline Lee1465 & 492.5 & -2.54 & -3.59 & 163.1 & 225.0 & 153.8 & 0.68 \\
\hline Lee 1540 & 633.6 & 0.1 & -0.71 & 175.2 & 133.4 & 142.5 & 1.07 \\
\hline Lee1544 & 486.9 & -2.51 & -3.54 & 153.9 & 146.8 & 142.0 & 0.97 \\
\hline
\end{tabular}

Notes: MW: Molecular weight

CLogP: Calculated logarithm of partition coefficient

LogD: Calculated logarithm of distribution coefficient at $\mathrm{pH} 7.0$

tPSA: Topological polar surface area

$\mathrm{P}_{\mathrm{app}}$ : Apparent permeability coefficient

B/A: Basolateral to apical

A/B: Apical to basolateral 
that these molecules are highly hydrophilic with the most lipophilic being Lee1540. All the active spectinamides were easily soluble in water (at least $50 \mathrm{mg} / \mathrm{mL}$ ). Due to insufficient compound, the exact solubility has not been reported.

The topological polar surface area (tPSA) is a physicochemical descriptor that is used in predicting permeability and a PSA $\leq 140 \AA^{2}$ indicates good gastrointestinal permeability leading to an oral bioavailability anywhere between $20-100 \%$ (140). However, all the active spectinamides synthesized so far had a PSA $>140 \AA^{2}$ suggesting poor permeability. This was evident in the Caco- 2 permeability assay which is often used as a surrogate for human intestinal permeability. All the spectinamides showed very low apparent permeability in the range of $\mathrm{nm} / \mathrm{s}$. All these results suggest that spectinamides have poor permeability and hence without any chemical modification would lead to poor oral bioavailability. However, they all can be administered via the intravenous route.

\section{In Vitro Microsomal Metabolic Stability}

Metabolic stability is an important property of a drug candidate since it affects the PK parameters such as hepatic clearance, half-life as well as bioavailability. Spectinomycin; spectinamides Lee1329, Lee1443, and Lee1445; nitrofuranylamide Lee1341 as positive control, with known significant metabolism by microsomal fraction, (unpublished report); and deactivated microsomes as negative control were studied in this assay. Spectinomycin and spectinamides were found to be metabolically stable to enzymes present in the microsomal fraction i.e., essentially CYP family of enzymes. After 90 min incubation in the presence of NADPH and rat liver microsomal fraction, the parent compound remaining was found to be between 80 to 100\% (Figure 3-2) indicating that the spectinamides do not undergo significant oxidation, reduction or hydrolysis catalyzed by Phase I enzymes. This also suggests that following oral administration, there will likely not be any significant first pass effect. This result indicates that the most likely route of elimination following i.v. administration would be via the renal route as seen in the case of spectinomycin where nearly $75 \%$ of the drug was eliminated unchanged in the urine (42). Table 3-2 shows the metabolic half-life of spectinamides in the presence of mouse and human liver microsomal fraction.

\section{Protein Binding}

Plasma protein binding can be an important factor in determining drug distribution, metabolism and elimination. Drugs usually are non-specifically adsorbed onto protein surfaces or reversibly bound at specific sites. Therefore, a drug in circulation will exist in two forms, bound and unbound and assuming that the binding is reversible, these two forms will be in equilibrium. It is only the unbound drug can diffuse across cell membranes and cause a pharmacological effect. Hence it is important to evaluate the extent of plasma protein binding. Apart from Lee1540, spectinamides in general showed low to intermediate protein binding in human, rat and mouse plasma. Lee1540 was 90\%$100 \%$ bound to plasma proteins which can be attributed to its improved lipophilicity 


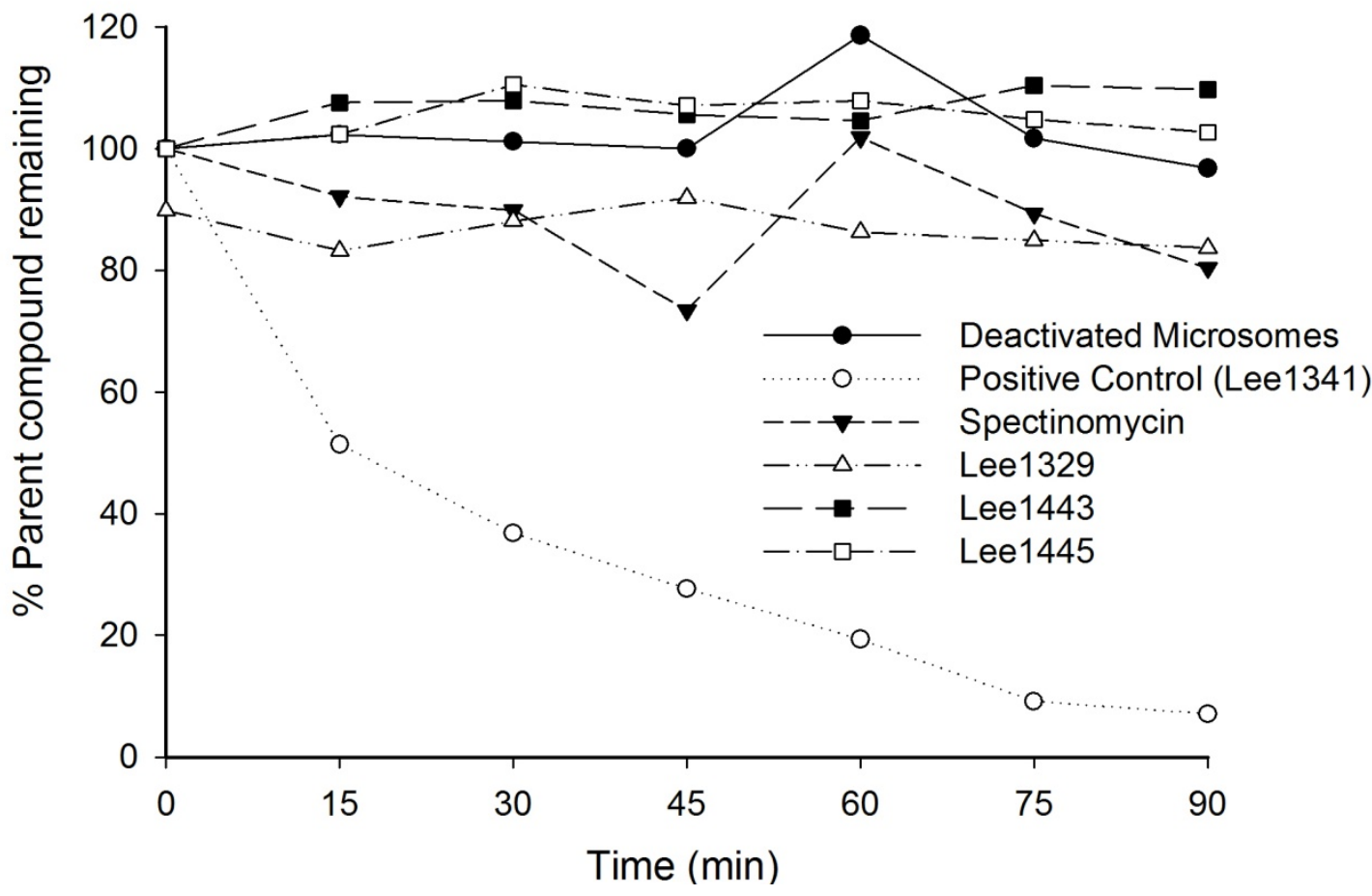

Figure 3-2. In vitro rat liver microsomal metabolic stability of spectinamides. 
Table 3-2. Metabolic half-life of spectinamides in presence of human and mouse liver microsomes.

\begin{tabular}{ccc}
\hline Compound & $\begin{array}{c}\text { Half-life (hr) } \\
\text { human }\end{array}$ & $\begin{array}{c}\text { Half-life (hr) } \\
\text { mouse }\end{array}$ \\
\hline Spectinomycin & 2.48 & $>10$ \\
Lee1329 & 8.59 & $>10$ \\
Lee1443 & 18.51 & 5.59 \\
Lee1445 & 5.24 & 6.89 \\
Lee1465 & 22.07 & 13.08 \\
Lee1540 & $>50$ & $>50$ \\
Lee1544 & 2.58 & 1.51 \\
\hline
\end{tabular}


compared to other spectinamides (CLogP of 0.1). Table 3-3 summarizes the extent of plasma protein binding of spectinamides in human, rat and mouse plasma. This result suggests that plasma protein binding does not limit the drug concentrations from reaching therapeutic levels intracellularly for its efficacy.

\section{Pharmacokinetics}

\section{First Generation Molecules}

Lee1329, Lee1443, Lee1445 and Lee1544 are 2-heteroaryl acetic acid amide analogs of spectinomycin and belong to the first generation of spectinamides that showed potent in vitro activity against MTB. Following intravenous administration of $10 \mathrm{mg} / \mathrm{Kg}$ in rats, all molecules except Lee1443 showed similar bi-exponential concentration-time profiles with distinct distribution and elimination phases (Figures 3-3, 3-4 and 3-5). The pharmacokinetic parameters are shown in Table 3-4. Due to the lack of a very sensitive analytical method for Lee1443, concentrations below $7.8 \mu \mathrm{g} / \mathrm{L}$ could not be quantified. Since Lee1443 concentrations beyond 4 hours fell below this LLOQ of $7.8 \mu \mathrm{g} / \mathrm{L}$, the biexponential PK profile could not be captured for this molecule. Hence a monoexponential concentration-time profile was fit to the available data.

All four compounds showed a similar alpha half-life of 0.30 to $0.35 \mathrm{hr}$. Lee1329, Lee1445 and Lee1544 showed a prolonged beta half-life ranging from 6.6 to $21 \mathrm{hr}$. The beta phase, however, contributed only minimally to the overall systemic exposure (AUC) with its plasma concentrations being 100 to 1000 fold lower than the peak concentrations. This behavior is common for aminoglycosides and many other amines and is likely related to slow leakage of compound from deep tissue compartments. The underlying mechanism is likely lysosomal trapping and/or complexation with membrane phospholipids (141).

First generation spectinamides showed a steady state volume of distribution ranging from 0.46 to $1.15 \mathrm{~L} / \mathrm{Kg}$ and the area under the curve ranged from 11.3 to 18 $\mathrm{mg} . \mathrm{hr} / \mathrm{L}$. They exhibited a mean residence time of $0.52-1.55$ hours and a mean systemic clearance of $0.56-0.89 \mathrm{~L} / \mathrm{hr} / \mathrm{Kg}$. The percent dose excreted unchanged by the kidneys $\left(\mathrm{f}_{\mathrm{e}}\right)$ ranged from $46 \%$ to a $100 \%$. The excretion ratio $\left(\mathrm{E}_{\text {ratio }}\right)$ for all these compounds was greater than one indicating active secretion as the net urinary elimination process.

Following $100 \mathrm{mg} / \mathrm{kg}$ oral administration in rats, Lee1329 and Lee1544 showed similar bi-exponential concentration-time profiles (Figures 3-3 and 3-5). However, they exhibited poor oral bioavailability of 4.0 and $3.2 \%$ respectively (Table 3-5). These molecules have limited permeability leading to poor oral bioavailability. Hence improving lipophilicity and decreasing polar surface area without compromising on the solubility might be a strategy to enhance the oral bioavailability of these agents. 
Table 3-3. Protein binding of spectinamides in human, rat and mouse plasma.

\begin{tabular}{cccc}
\hline Compound & $\begin{array}{c}\text { \%Bound } \\
\text { human }\end{array}$ & $\begin{array}{c}\text { \%Bound } \\
\text { rat }\end{array}$ & $\begin{array}{c}\text { \%Bound } \\
\text { mouse }\end{array}$ \\
\hline Spectinomycin & 51.6 & - & 52.6 \\
Lee1329 & 62.1 & 28.5 & 36.9 \\
Lee1443 & 52.4 & 13.0 & 41.6 \\
Lee1445 & 45.8 & 28.0 & 38.0 \\
Lee1465 & 42.9 & 44.0 & 50.0 \\
Lee1540 & 96.9 & 90.0 & 100.0 \\
Lee1544 & 56.4 & 15.0 & 39.8 \\
\hline
\end{tabular}


A)

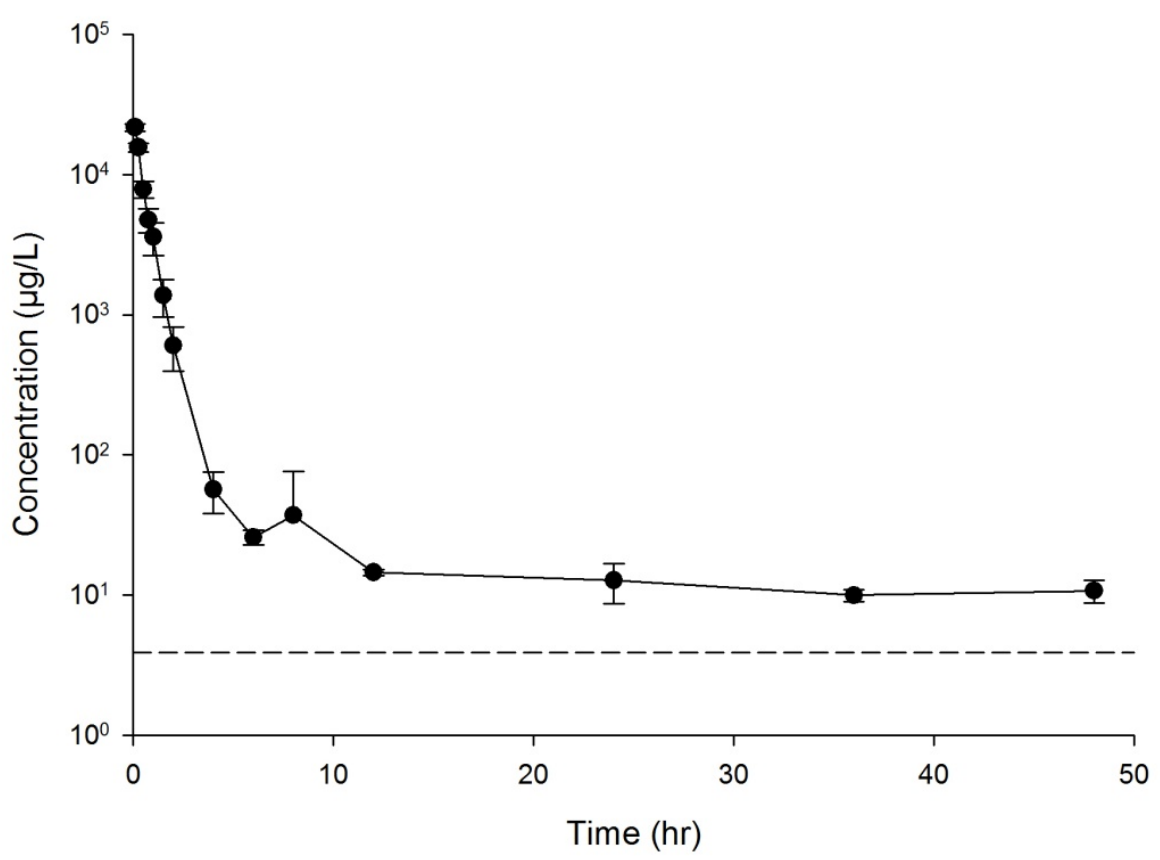

B)

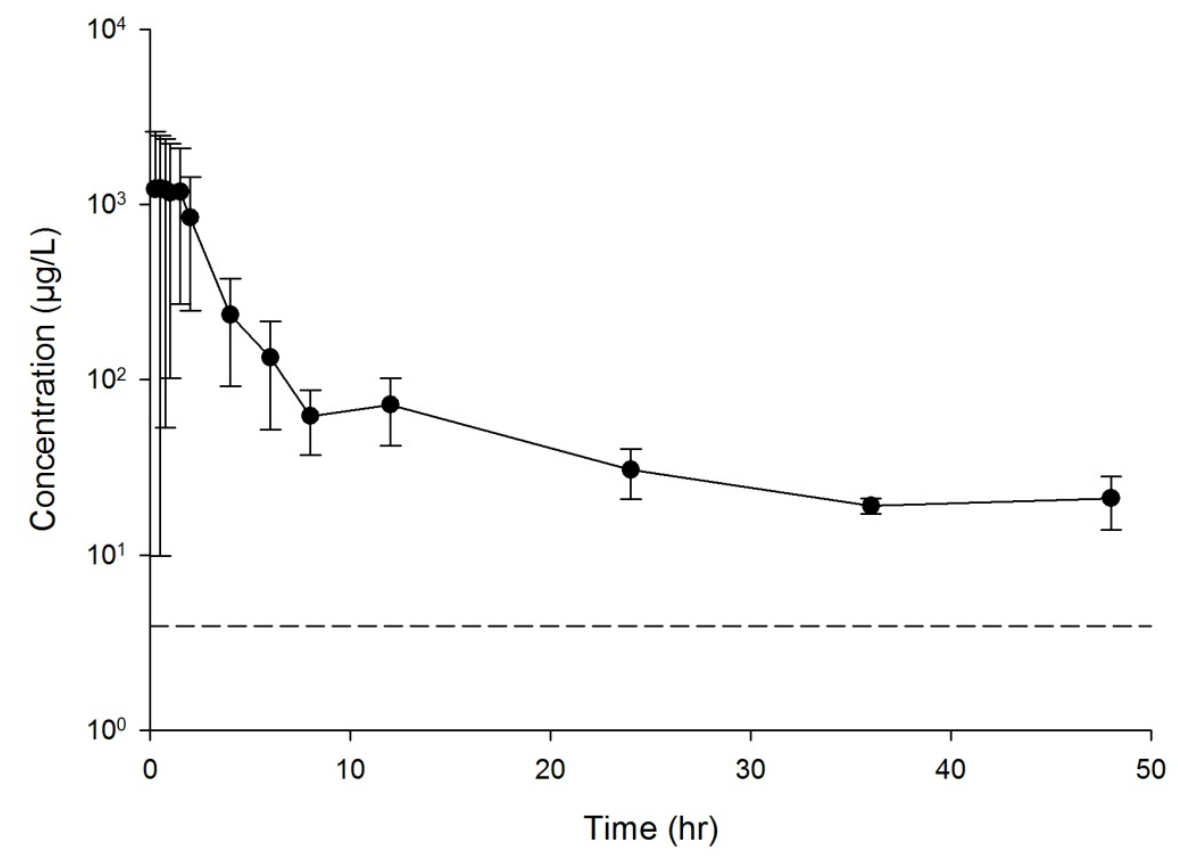

Figure 3-3. Measured plasma concentration-time profiles (mean \pm SD) after intravenous $(10 \mathrm{mg} / \mathrm{Kg}$, Panel A) or oral $(100 \mathrm{mg} / \mathrm{Kg}$, Panel B) administration of Lee1329 in rats. Dashed line denotes LLOQ. 
A)

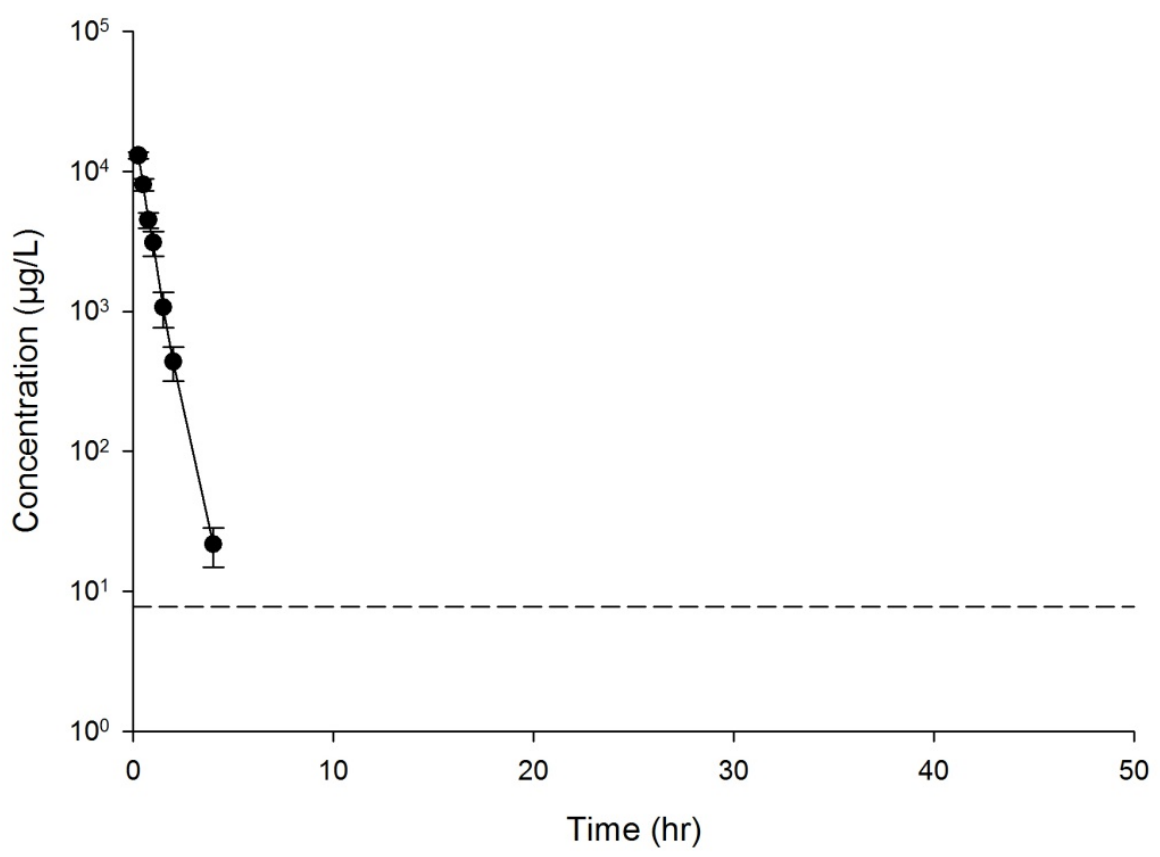

B)

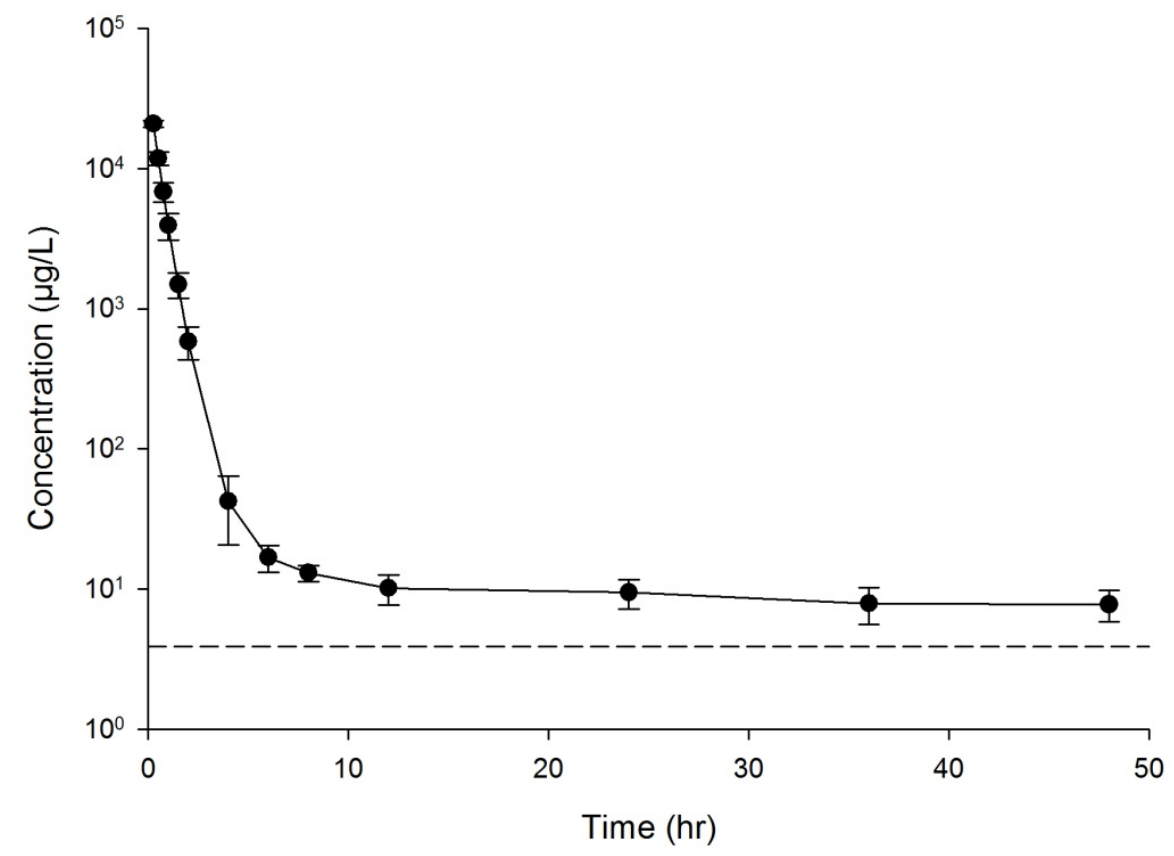

Figure 3-4. Measured plasma concentration-time profiles (mean $\pm \mathrm{SD}$ ) after $10 \mathrm{mg} / \mathrm{Kg}$ intravenous administration of Lee1443 (Panel A) and Lee1445 (Panel B) in rats. Dashed line denotes LLOQ. 
A)

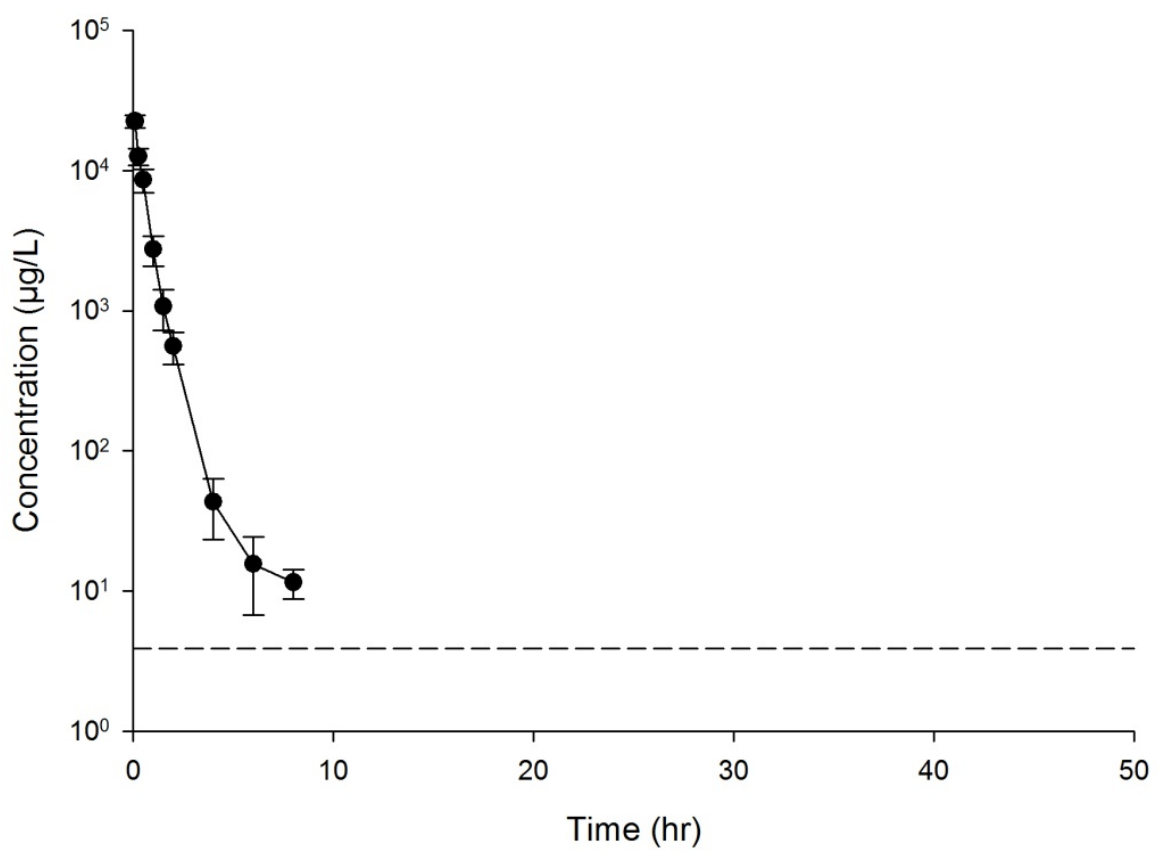

B)

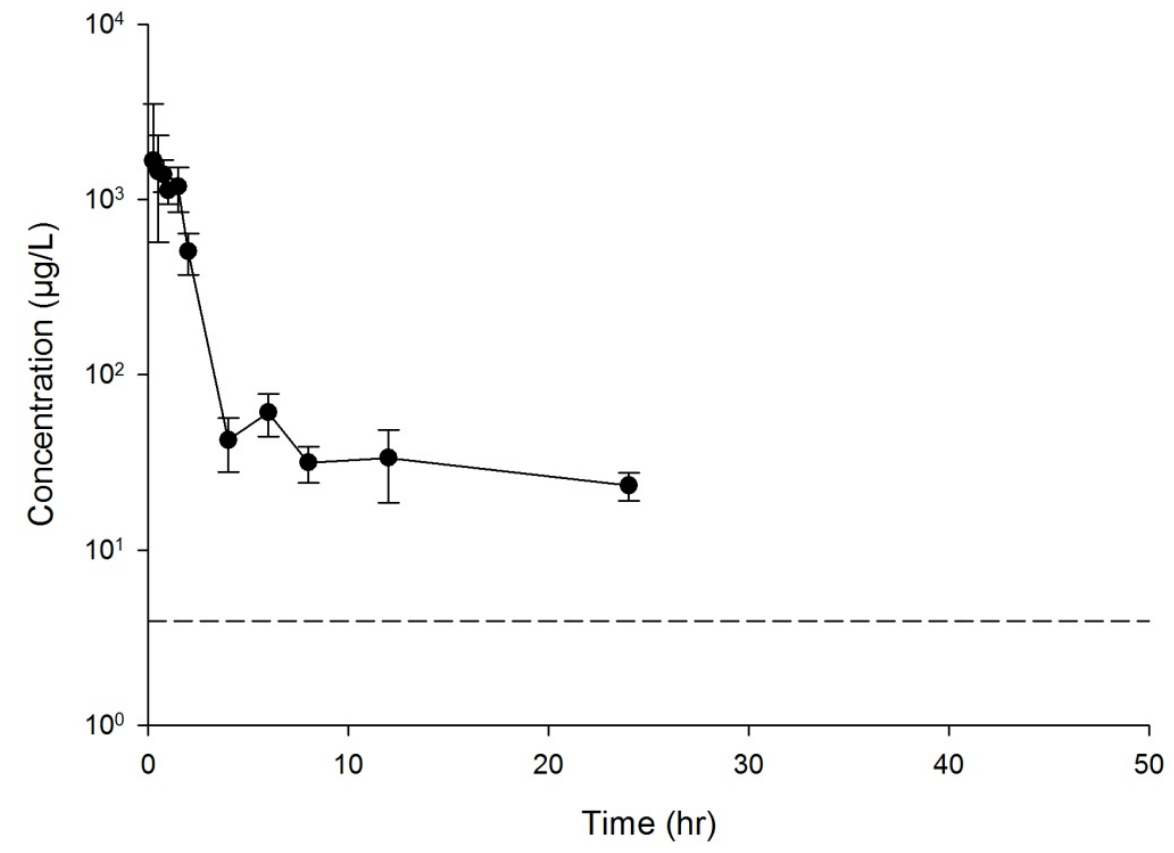

Figure 3-5. Measured plasma concentration-time profiles (mean \pm SD) after intravenous $(10 \mathrm{mg} / \mathrm{Kg}$, Panel A) or oral $(100 \mathrm{mg} / \mathrm{Kg}$, Panel B) administration of Lee1544 in rats. Dashed line denotes LLOQ. 
Table 3-4. Pharmacokinetic parameters of spectinamides following intravenous administration (10 mg/Kg).

\begin{tabular}{|c|c|c|c|c|c|c|c|c|c|c|}
\hline Compound & $\begin{array}{c}\mathbf{C}_{\max } \\
(\mathrm{mg} / \mathrm{L})\end{array}$ & $\begin{array}{c}\mathbf{A U C}_{\text {inf }} \\
(\mathrm{mg} \cdot \mathrm{hr} / \mathrm{L})\end{array}$ & $\begin{array}{c}\mathbf{C L} \\
(\mathrm{L} / \mathrm{hr} / \mathrm{Kg})\end{array}$ & $\begin{array}{c}\mathbf{V d} \\
(\mathrm{L} / \mathrm{Kg})\end{array}$ & $\begin{array}{l}\mathbf{t}_{1 / 2} \\
(\mathrm{hr})\end{array}$ & $\begin{array}{l}\mathbf{t}_{1 / 2 \alpha} \\
(\mathrm{hr})\end{array}$ & $\begin{array}{l}\mathbf{t}_{1 / 2 \beta} \\
(\mathrm{hr})\end{array}$ & $\begin{array}{c}\text { MRT } \\
(\mathrm{hr})\end{array}$ & $\mathbf{f}_{\mathrm{e}}$ & $\mathbf{E}_{\text {ratio }}$ \\
\hline Lee1329 & $21.6 \pm 1.3$ & $13.8 \pm 1.4$ & $0.74 \pm 0.08$ & $1.15 \pm 0.14$ & $0.48 \pm 0.04$ & $0.34 \pm 0.05$ & $15.3 \pm 3.6$ & $1.55 \pm 0.05$ & $0.46 \pm 0.04$ & 1.4 \\
\hline Lee1443 & $13.0 \pm 0.7$ & $11.3 \pm 0.8$ & $0.89 \pm 0.06$ & $0.46 \pm 0.05$ & $0.44 \pm 0.01$ & $0.35 \pm 0.05$ & - & $0.52 \pm 0.06$ & $0.87 \pm 0.11$ & 2.9 \\
\hline Lee1445 & $20.9 \pm 1.2$ & $18.0 \pm 1.6$ & $0.56 \pm 0.05$ & $1.03 \pm 0.13$ & $0.43 \pm 0.06$ & $0.32 \pm 0.03$ & $21.0 \pm 7.8$ & $0.57 \pm 0.04$ & $1.08 \pm 0.09$ & 2.5 \\
\hline Lee1465 & $24.2 \pm 5.2$ & $19.9 \pm 4.4$ & $0.53 \pm 0.15$ & $0.36 \pm 0.15$ & $0.54 \pm 0.07$ & $0.30 \pm 0.03$ & $14.2 \pm 7.4$ & $0.68 \pm 0.16$ & $0.99 \pm 0.06$ & 2.1 \\
\hline Lee 1540 & $35.8 \pm 11.2$ & $31.7 \pm 10.8$ & $0.36 \pm 0.16$ & $0.27 \pm 0.15$ & $0.62 \pm 0.09$ & $0.31 \pm 0.02$ & $12.5 \pm 6.1$ & $0.73 \pm 0.28$ & $0.06 \pm 0.04$ & 0.49 \\
\hline Lee1544 & $22.4 \pm 2.4$ & $12.9 \pm 1.4$ & $0.78 \pm 0.08$ & $0.80 \pm 0.47$ & $0.56 \pm 0.08$ & $0.30 \pm 0.03$ & $6.6 \pm 7.3$ & $1.02 \pm 0.59$ & $0.62 \pm 0.25$ & 1.5 \\
\hline
\end{tabular}

Notes: $\mathrm{C}_{\max }$ : Peak or maximum concentration

$\mathrm{AUC}_{\text {inf: }}$ : Area under the curve from time zero extrapolated to infinity

CL: Total body clearance

$\mathrm{Vd}$ : Volume of distribution

$\mathrm{t}_{1 / 2}$ : Half-life

$t_{1 / 2 \alpha}$ : Half-life associated with the macro rate constant $\alpha$

$t_{1 / 2 \beta}$ : Half-life associated with the macro rate constant $\beta$

MRT: Mean residence time

$\mathrm{f}_{\mathrm{e}}$ : Fraction of drug eliminated unchanged in the urine

$\mathrm{E}_{\text {ratio }}$ : Excretion ratio 
Table 3-5. Pharmacokinetic parameters of spectinamides following oral administration $(100 \mathrm{mg} / \mathrm{Kg})$.

\begin{tabular}{ccccc}
\hline Compound & $\begin{array}{c}\mathbf{C}_{\max } \\
(\mathrm{mg} / \mathrm{L})\end{array}$ & $\begin{array}{c}\mathbf{T}_{\max } \\
(\mathrm{hr})\end{array}$ & $\begin{array}{c}\mathbf{A U} \mathbf{C}_{\mathbf{i n f}} \\
(\mathrm{mg} . \mathrm{hr} / \mathrm{L})\end{array}$ & $\begin{array}{c}\mathbf{F} \\
(\%)\end{array}$ \\
\hline Lee1329 & $1.46 \pm 1.24$ & $0.69 \pm 0.59$ & $5.26 \pm 3.19$ & 4.0 \\
Lee1465 & $1.38 \pm 0.37$ & $0.56 \pm 0.24$ & $2.36 \pm 0.64$ & 1.2 \\
Lee1540 & $1.94 \pm 0.95$ & $0.58 \pm 0.14$ & $2.87 \pm 0.83$ & 0.9 \\
Lee1544 & $2.14 \pm 1.56$ & $0.75 \pm 0.47$ & $4.12 \pm 0.82$ & 3.2 \\
\hline
\end{tabular}

Notes: $\mathrm{C}_{\text {max }}$ : Peak or maximum concentration

$\mathrm{T}_{\max }$ : Time to reach peak concentration

$\mathrm{AUC}_{\text {inf: }}$ : Area under the curve from time zero extrapolated to infinity

F: Absolute bioavailability 


\section{Second Generation Molecules}

Fused ring analog Lee1465 and phenyl amino thiazole analog Lee1540 belong to the second generation of spectinamides that were found to be active in the in vitro assays against MTB. Following i.v. administration (Figure 3-6), Lee1465 had a mean systemic clearance of $0.53 \mathrm{~L} / \mathrm{hr} / \mathrm{Kg}$ and exhibited a steady state volume of distribution of 0.36 $\mathrm{L} / \mathrm{Kg}$. It is only $40 \%$ bound to plasma proteins and has a good microsomal metabolic stability. Therefore most of the dose was eliminated unchanged by the kidneys $\left(f_{e} 0.99\right)$. Thus, renal excretion is the major elimination pathway for this molecule. The excretion ratio $\left(\mathrm{E}_{\text {ratio }}\right)$ of Lee1465 is 2.1 suggesting active secretion as the net urinary elimination process. In contrast, Lee1540 is characterized by a high plasma protein binding of $90 \%$ $100 \%$, probably a reflection of its increased lipophilicity. Following intravenous administration (Figure 3-7), the mean systemic clearance of Lee1540 $(0.36 \mathrm{~L} / \mathrm{hr} / \mathrm{Kg})$ is low when compared to the other compounds in the series and it has a relatively low steady state volume of distribution of $0.27 \mathrm{~L} / \mathrm{Kg}$ indicative of its poor tissue permeability. The fraction of dose excreted unchanged by the kidneys $\left(f_{e}\right)$ is $6 \%$. Thus, non-renal elimination seems to be the major elimination pathway for Lee1540. Since microsomal metabolic stability studies suggest high stability towards liver microsomes from mice, rats and humans, the elimination of Lee1540 is likely related to phase II metabolism and/or biliary excretion. Also, Lee1540 has a MW of 633.6 Da and the likelihood of phase II metabolism increases with MW for compounds greater than $500 \mathrm{Da}$.

Following oral administration, both Lee1465 and Lee1540 showed very poor oral bioavailability ( 1.2 and $0.9 \%$ respectively) in spite of their improved lipophilicity in relation to other molecules in the series. This can be attributed to the fact that the chemical modifications increased the polar surface area of the molecules making them even more poorly permeable. Based on the bioavailability findings, all these agents can be administered via intravenous route until newer compounds with much better oral bioavailability are discovered.

\section{Drug Uptake into M. bovis BCG}

Spectinamides and spectinomycin are structurally similar molecules. However, first generation spectinamides are approximately 30-60 times more potent than spectinomycin in the in vitro $\mathrm{MIC}$ assays (Figure 3-1). Interestingly, the $\mathrm{IC}_{50}$ values for the antiribosomal activity in a cell free translation assay using $M$. smegmatis ribosomes did not significantly differ between the spectinamides and spectinomycin indicating that all these have similar intrinsic activity at the target site on the ribosome. In order to evaluate the differences in their potencies, drug uptake into mycobacterium was studied. After incubating the $M$. bovis BCG cells with different molecules for a period of 4 hours, the total drug taken up into the cells was estimated. The results of this assay are shown in Table 3-6.

Compared to spectinomycin, spectinamides Lee1329 and Lee1445 showed approximately 4 times higher uptake into the mycobacterial cells. Since these molecules 
A)

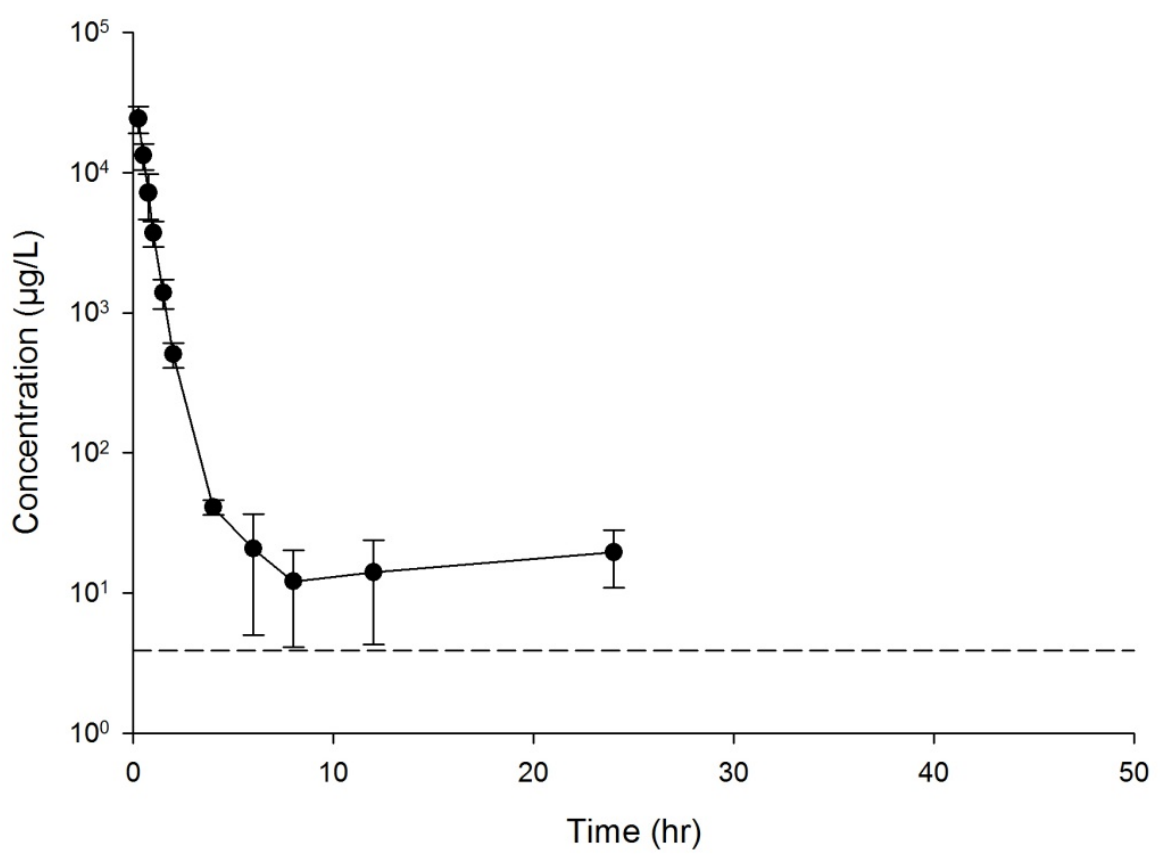

B)

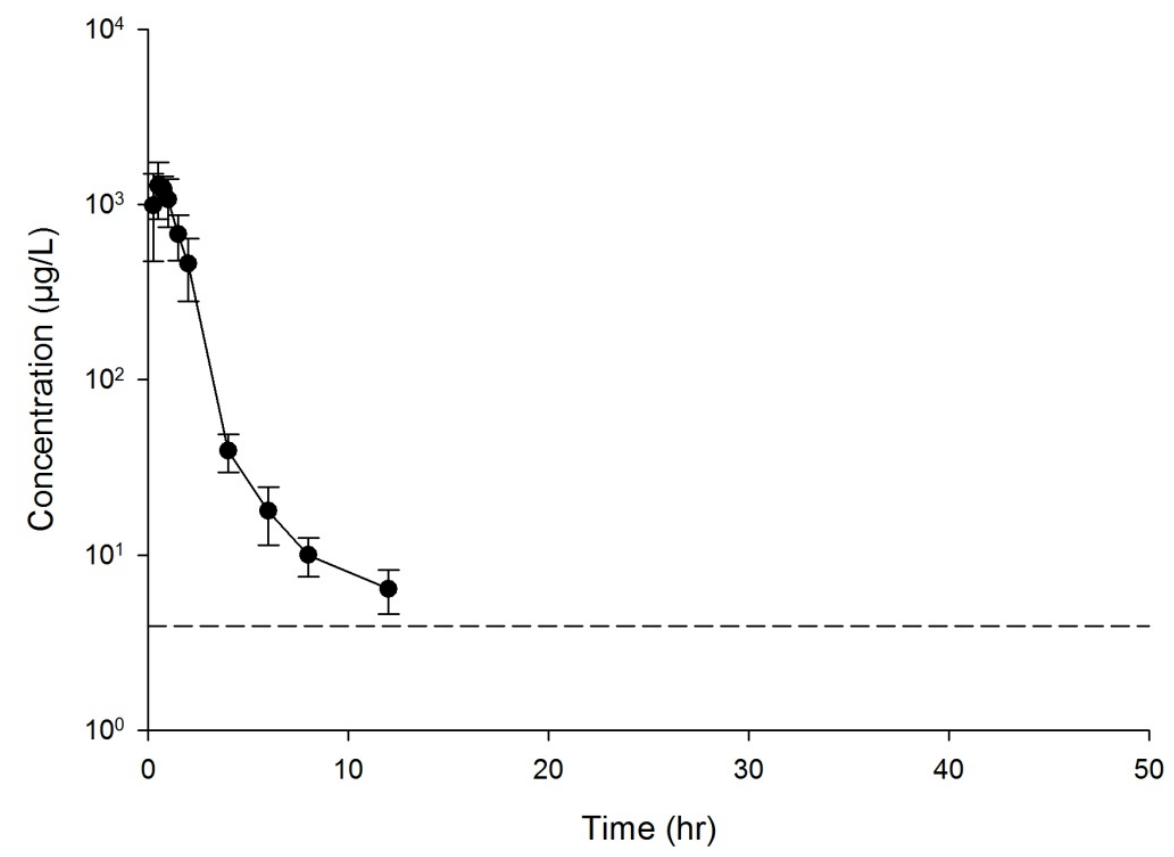

Figure 3-6. Measured plasma concentration-time profiles (mean \pm SD) after intravenous $(10 \mathrm{mg} / \mathrm{Kg}$, Panel A) or oral $(100 \mathrm{mg} / \mathrm{Kg}$, Panel B) administration of Lee1465 in rats. Dashed line denotes LLOQ. 
A)

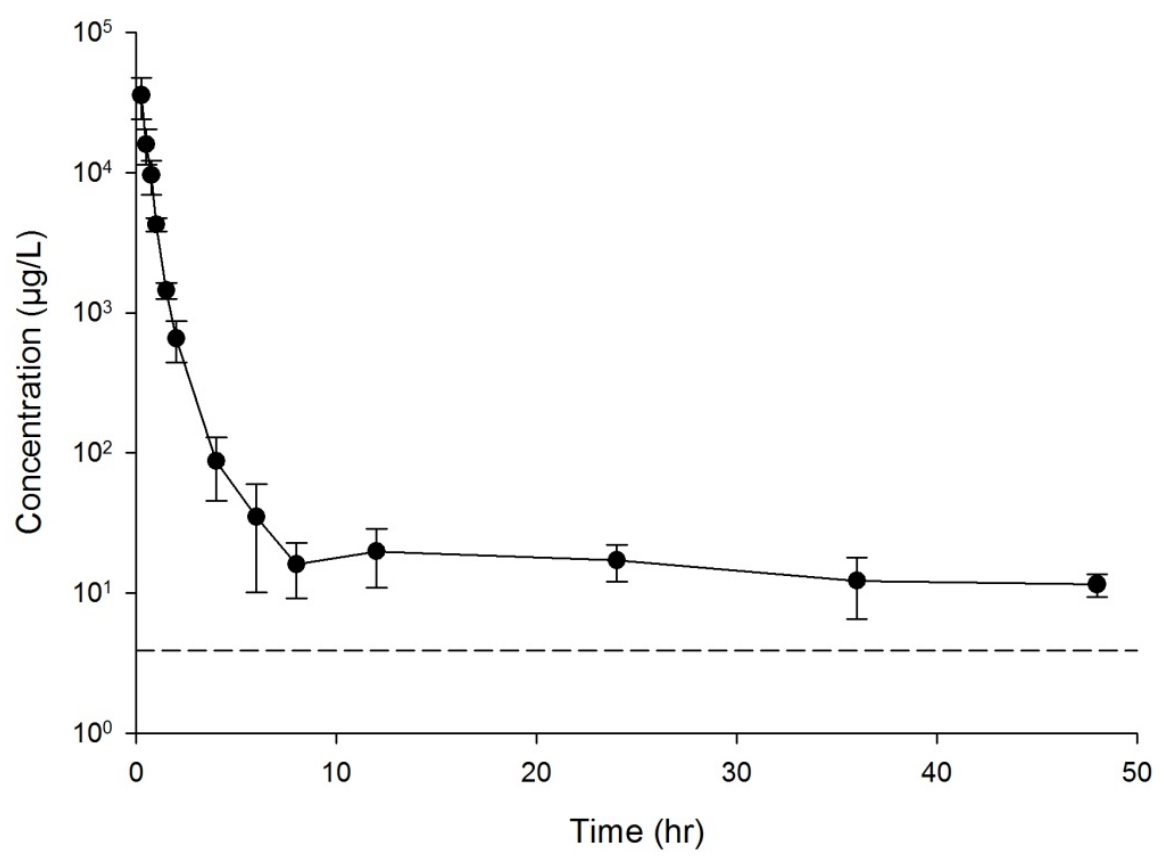

B)

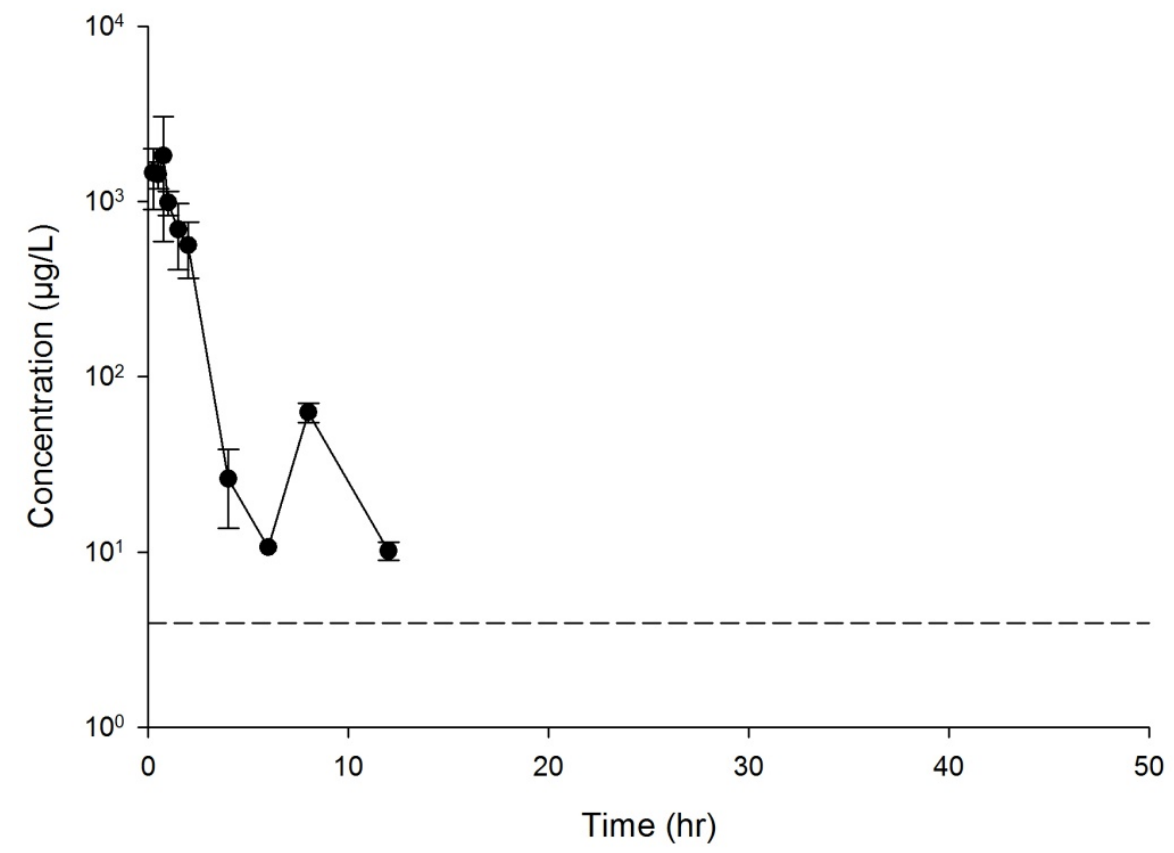

Figure 3-7. Measured plasma concentration-time profiles (mean $\pm \mathrm{SD}$ ) after intravenous (10 mg/Kg, Panel A) or oral $(100 \mathrm{mg} / \mathrm{Kg}$, Panel B) administration of Lee1540 in rats. Dashed line denotes LLOQ. 
Table 3-6. Drug uptake into M. bovis BCG.

\begin{tabular}{cccc}
\hline Molecule & $\begin{array}{c}\text { Amount (ng)/ } \\
\text { weight of cells (gm) }\end{array}$ & \%CV & $\begin{array}{c}\text { Ratio w.r.t. } \\
\text { spectinomycin }\end{array}$ \\
\hline Spectinomycin & 650 & 5 & - \\
Lee1329 & 2548 & 3.6 & 3.9 \\
Lee1445 & 2412 & 3.6 & 3.7 \\
\hline
\end{tabular}

Note: CV: Coefficient of variation 
have similar solubility, lipophilicity, and tissue permeability, it was hypothesized that some active efflux pumps operate on the mycobacterial envelope that selectively pump out spectinomycin but not spectinamides thereby higher concentrations prevail for spectinamides. MIC studies performed by our collaborators at Universidad de Zaragoza, Spain, on efflux pump knockouts (KO-Rv1258c, KO-Rv1410c, KO-Rv2333c, KORv3065) of MTB showed that spectinamides Lee1329, Lee1445, and Lee1544 are not substrates to any of these efflux pumps while spectinomycin is a good substrate of efflux pump encoded by Rv1258c gene (unpublished report). The results of this study clearly answered some of the mechanistic aspects related to the potency of spectinamides in comparison to spectinomycin.

\section{Drug Uptake into Murine Macrophages}

Before addition of the drug to the cells, cytotoxicity of spectinamides was assessed to ensure that drug treatment does not lyse the cells compromising the cell integrity. This was performed using an MTT cell proliferation assay for Lee1329, Lee1443, Lee1445 and streptomycin. The results of this assay are shown in Figure 3-8. These results indicate that spectinamides did not possess cytotoxicity towards $\mathrm{J} 774$ murine macrophage cells even at very high concentrations of $200 \mu \mathrm{g} / \mathrm{mL}$ and hence ensures that the quantified drug would be from the intact macrophages and not from the macrophages lysed due to cell cytotoxicity.

After being ingested by macrophages, the mycobacteria continue to multiply slowly with bacterial cell division occurring every 25 to 32 hours (142). Therefore it is important that the antimycobacterial agents reach the site where bacilli pervade within macrophages. To evaluate this, spectinamides were incubated with murine macrophages and the drug uptake into macrophages was quantified. Streptomycin, a well established second line antituberculosis agent was used as a control. The results of this assay are shown in Table 3-7. Compared to streptomycin, spectinamides Lee1329, Lee1443, and Lee1445 had 3 to 4 times better uptake into macrophages. These results suggest that sufficient intracellular concentrations of spectinamides can be attained within macrophages that can target the primary TB infection.

\section{Conclusion}

Identification and characterization of lead molecules from this spectinamide series of antituberculosis agents was achieved via a screening paradigm consisting of a series of existing in vivo and in vitro assays such as plasma protein binding, microsomal metabolic stability and in vivo pharmacokinetics, and novel drug uptake assays into M. bovis BCG and J774 murine macrophages. Spectinamides exhibited low to intermediate plasma protein binding and were found to be metabolically stable with the exception of Lee1540. Following intravenous administration, spectinamides were widely distributed ( 0.36 to $1.15 \mathrm{~L} / \mathrm{Kg}$ ) with short half-lives $(0.43$ to $0.62 \mathrm{hr}$ ). Mean systemic clearance ranged between 0.36 to $0.89 \mathrm{~L} / \mathrm{hr} / \mathrm{Kg}$ with a significant fraction of drug eliminated unchanged in urine ( 0.46 to 1.0$)$. Spectinamides except Lee1540 exhibited a renal excretion ratio 


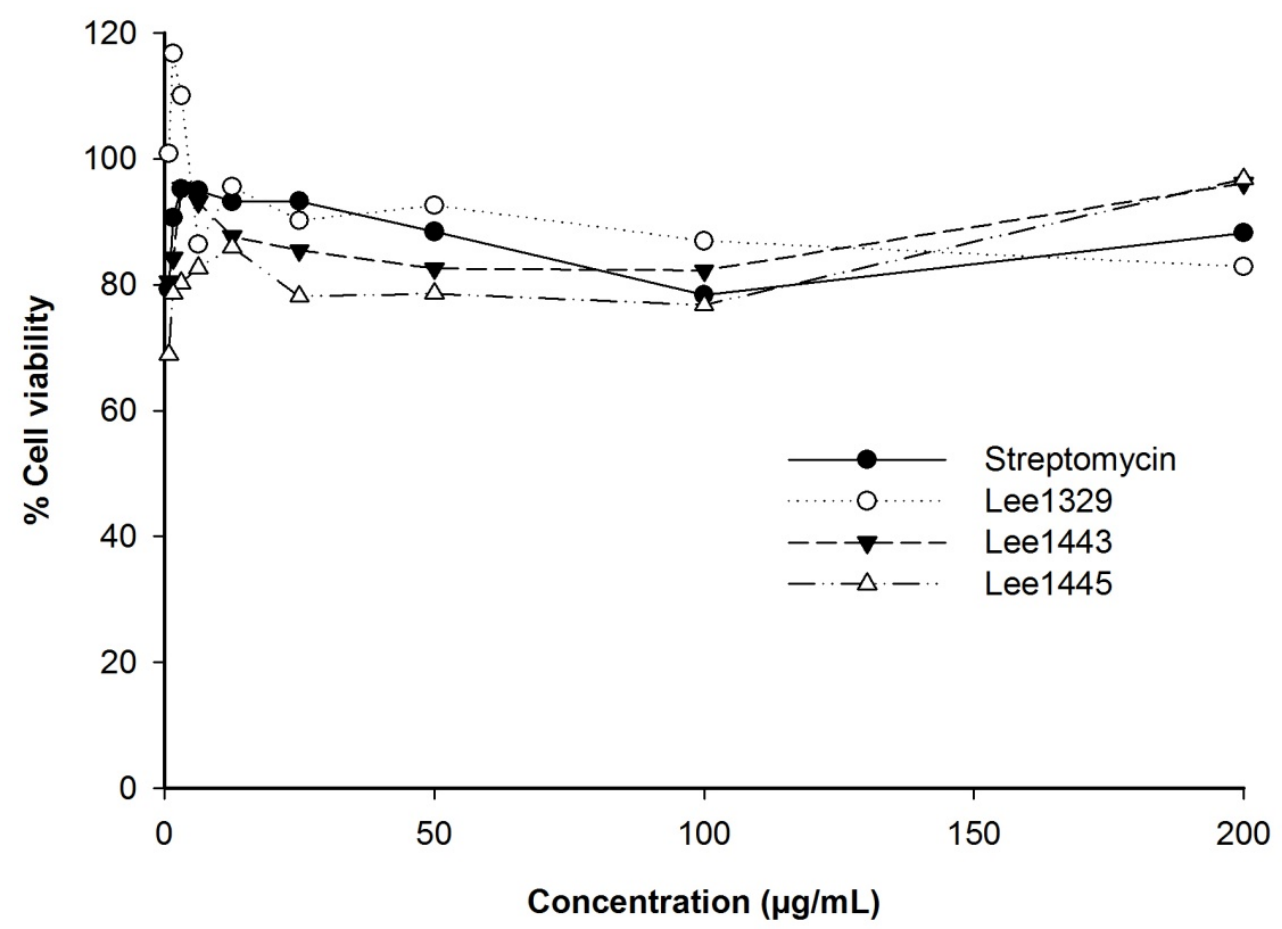

Figure 3-8. Cytotoxicity of spectinamides against J774 murine macrophages. 
Table 3-7. Drug uptake into the J774 murine macrophage cell line.

\begin{tabular}{cccc}
\hline Molecule & $\begin{array}{c}\text { Amount (ng)/ } \\
\text { weight of cells (gm) }\end{array}$ & $\mathbf{\% C V}$ & $\begin{array}{c}\text { Ratio w.r.t. } \\
\text { streptomycin }\end{array}$ \\
\hline Streptomycin & 10303 & 6.2 & - \\
Lee1329 & 29126 & 5.7 & 2.8 \\
Lee1443 & 38450 & 5.4 & 3.7 \\
Lee1445 & 44299 & 6.6 & 4.3 \\
\hline
\end{tabular}

Note: CV: Coefficient of variation 
greater than 1 indicating filtration and active secretion as the net renal elimination process. In the uptake experiments, the spectinamides exhibited 3-4 times higher uptake into murine macrophages compared to streptomycin and showed nearly four times higher uptake into M. bovis BCG compared to spectinomycin, which may in part explain their increased activity compared to spectinomycin.

Based on these properties, new generation of molecules can be synthesized retrospectively via structural modification to further improve their PK properties. In conclusion, we characterized the pharmacokinetic properties of key compounds in the spectinamide series and developed a screening paradigm that could be used for further optimization of leads specifically against MTB. 


\section{CHAPTER 4. PHARMACODYNAMIC EVALUATION AND DOSE OPTIMIZATION OF LEE1445 USING AN IN VITRO PK/PD MODEL}

\section{Introduction}

The MIC is the most commonly used parameter to describe the efficacy of an antibacterial agent against a bacterial strain. However, it is not an optimal pharmacodynamic marker because it reflects only a point estimate of the effect and does not take the time course of the effect into account. The design of dosing schedules can further be optimized if they are based on models that take the whole time course of the $\mathrm{PK} / \mathrm{PD}$ relationship, i.e., the time course of both the drug concentration and the bacterial concentration, into consideration (128). In vitro experiments are useful in evaluating such direct interaction between the drug and the bacteria, which enables the dose selection of candidate compounds. Utilizing in vivo drug clearance profiles from animal and/or human studies and simulating them in an in vitro PK/PD model allows the in-depth characterization of antimycobacterial activity of anti-TB agents by generating time-kill data. These data capture the dynamic interplay between mycobacterial growth and changing drug concentration as encountered during prolonged drug therapy (137).

Spectinamides are a novel class of antituberculosis agents with potent in vitro antituberculosis activity that have been recently characterized by our group. On the basis of the pharmacokinetically guided screening paradigm, discussed in Chapter 2, which is designed to identify molecules with most suitable properties to become an effective antituberculosis agent, Lee1445 has emerged to be one such molecule from the class of spectinamides synthesized so far. Lee1445 was found to have potent in vitro activity against $M$. tuberculosis with an MIC of $0.4 \mu \mathrm{g} / \mathrm{mL}$ and an $\mathrm{IC}_{50}$ value of $0.58 \mu \mathrm{g} / \mathrm{mL}$ in the antiribosomal cell free translation assay using $M$. smegmatis ribosomes. From a PK perspective, it exhibited good metabolic stability with a half-life of $5.24 \mathrm{hr}$ in the presence of human liver microsomes. The stability of Lee1445 in liver microsomal preparations indicates no involvement of the hepatic CYP enzyme system in the clearance of the compound. This absence of major hepatic CYP mediated metabolism increases the likelihood of compatibility with existing anti HIV medications which are mainly metabolized by the hepatic CYP enzyme system and are frequently coadministered with anti-tuberculosis agents $(143,144)$. It has a protein binding of about $28 \%$ in rat plasma suggesting that protein binding does not limit the drug concentrations from reaching therapeutic levels intracellularly for its efficacy. It showed approximately 3 times higher uptake into mycobacteria compared to spectinomycin and about 4 times better uptake into murine macrophages compared to the existing second line agent streptomycin. Moreover, it did not possess any cell cytotoxicity against murine macrophages even at concentrations up to $200 \mu \mathrm{g} / \mathrm{mL}$. On the down side, it showed a relatively short half-life of $0.43 \mathrm{hr}$ with a clearance of $1.03 \mathrm{~L} / \mathrm{Kg}$ after i.v. administration in rats suggesting the need for frequent dosing. Spectinamides in general showed poor oral bioavailability. A prodrug approach is currently being explored to overcome this limitation. However, given its potency against MTB, Lee1445 can be administered via i.v. route and can still serve as a valuable therapeutic option. 
In the current chapter, we investigated the time course of bacterial kill of Lee1445 using a previously reported in vitro PK/PD system (19). A novel and rapid method to quantify the bacterial counts based on adenosine triphosphate (ATP) measurements was developed. PK concentration-time profiles were simulated on the basis of the in vivo clearance of rats and the time-kill effect of different daily doses of Lee1445 were studied on the growing $M$. bovis $\mathrm{BCG}$ present in the PK/PD system. A mathematical model was fit to the time-kill data and in vitro PK/PD parameters were determined. Since the in vivo efficacy model of tuberculosis infection used in our development program was the gamma knock-out (GKO) mouse, a PK bridging study was performed in mice.

Simulations for various dosing regimens were performed using the obtained mouse PK parameters and the in vitro $\mathrm{PK} / \mathrm{PD}$ parameters, which were incorporated into a combined $\mathrm{PK} / \mathrm{PD}$ model to predict the efficacy in terms of reduction of bacterial counts. Based on the results of the simulations, an optimal dose was chosen for the in vivo efficacy study.

\section{Materials and Methods}

\section{Culture, Media, and Antibiotics}

Mycobacterium bovis BCG was grown in Middlebrook 7H9 broth (Becton Dickinson, Sparks, MD) supplemented with $10 \%$ albumin dextrose complex and $0.1 \%$ $\mathrm{v} / \mathrm{v}$ Tween 80 . The cultures were stored at $-80^{\circ} \mathrm{C}$ in Middlebrook $7 \mathrm{H} 9$ broth. For each experiment, aliquots were thawed and incubated at $37^{\circ} \mathrm{C}$ in Middlebrook $7 \mathrm{H} 9$ broth for 4 days. The test compound Lee1445 (3'-dihydro-3'-deoxy-4(R)-(5-fluoropyridin-2-yl) acetylamino spectinomycin) shown in Figure 3-1, was synthesized in the laboratory of Dr. R.E. Lee at the St. Jude Children's Research Hospital, Memphis, TN. A stock solution of Lee1445 was prepared in methanol and stored at $-80^{\circ} \mathrm{C}$. Working solutions of the compound were prepared from the stock solution using distilled water.

\section{Minimum Inhibitory Concentration of Lee1445}

The MIC of Lee1445 was determined using the microbroth dilution method according to the CLSI guidelines (12) and was read by visual inspection. Two fold serial dilutions of Lee1445 in $100 \mu \mathrm{L}$ of the Middlebrook 7H9 broth medium were prepared in round-bottomed micro liter plates (Nunc, Sigma-Aldrich, St. Louis, MO). An equivalent volume $(100 \mu \mathrm{L})$ of broth containing $\sim 10^{5} \mathrm{CFU} / \mathrm{mL}$ bacteria was added to each well to give final concentration of Lee1445 starting at $200 \mathrm{mg} / \mathrm{L}$ and the plates were incubated aerobically at $37^{\circ} \mathrm{C}$ for 7 days. The MIC was recorded as the lowest concentration of the compound that prevented visible growth. 


\section{In Vitro PK/PD Model}

A previously reported in vitro PK/PD model system (19) was used which consisted of a two-armed, water jacketed spinner culture flask (1965 series spinner flask, Bellco Glass, Vineland, NJ). A prefilter $(5 \mu \mathrm{m}$, Millipore, Billerica, MA) and a filter membrane $(0.22 \mu \mathrm{m}$, Millipore, Billerica, MA) were placed in a $25 \mathrm{~mm}$ diameter filter unit (Pall Corporation, East Hills, NY) and this filter unit which is used to prevent leakage of bacteria during the dilution process, was fixed to one end of a custom made hollow steel tube. The other end of the steel tube was connected to plastic tubing (PharMed tubing, Cole-Parmer, Vernon Hills, IL). This whole filter unit was suspended into the media from the top. To ensure homogeneity of the culture and prevent blockage of the filter membrane, this flask set up was placed on a magnetic stirrer with a magnetic stir bar in the flask. The temperature in the flask was maintained at $37^{\circ} \mathrm{C}$ using a thermostat controlled water circulator, circulating water to the external jacket of the flask.

One of the side arms of the flask covered with silicone septa was used for dosing and repeated sampling while the other side arm was connected to a reservoir containing antibiotic free sterile medium through a thin plastic tube (PharMed tubing, Cole-Parmer, Vernon Hills, IL). The outlet tubing from the filter unit was connected to a peristaltic pump (Masterflex L/S, Cole-Parmer, Vernon Hills, IL) to continuously withdraw the medium from the flask at a constant rate. Fresh sterile medium (Middlebrook 7H9, Becton Dickinson, Sparks, MD) was pumped into the flask at the same rate.

Figure 4-1 shows the working picture of the in vitro PK/PD system. The solution containing the antibiotic Lee1445 was added through the side arm. Lee1445

concentration in the flask was decreased exponentially according to the equation,

$$
C=C_{0} \cdot e^{-k t}
$$

where $\mathrm{C}_{0}$ is the initial concentration of Lee1445 in the flask, $\mathrm{C}$ is the concentration at any time $\mathrm{t}, \mathrm{k}$ is the elimination rate constant and $\mathrm{t}$ is the time elapsed since the addition of Lee1445. The in vivo half-life $\left(\mathrm{t}_{1 / 2}\right)$ of Lee1445 was simulated in the model by changing the flow rate according to the equation,

$$
F=V \cdot \frac{\ln 2}{t_{1 / 2}}
$$

where the elimination rate constant $\mathrm{k}$ is equal to $\mathrm{F} / \mathrm{V}$, where $\mathrm{F}$ is the flow rate of the medium and $\mathrm{V}$ is the volume of the medium in the flask. The apparatus was sterilized by autoclaving between experiments and was kept in a biological safety cabinet during operation. 
A)

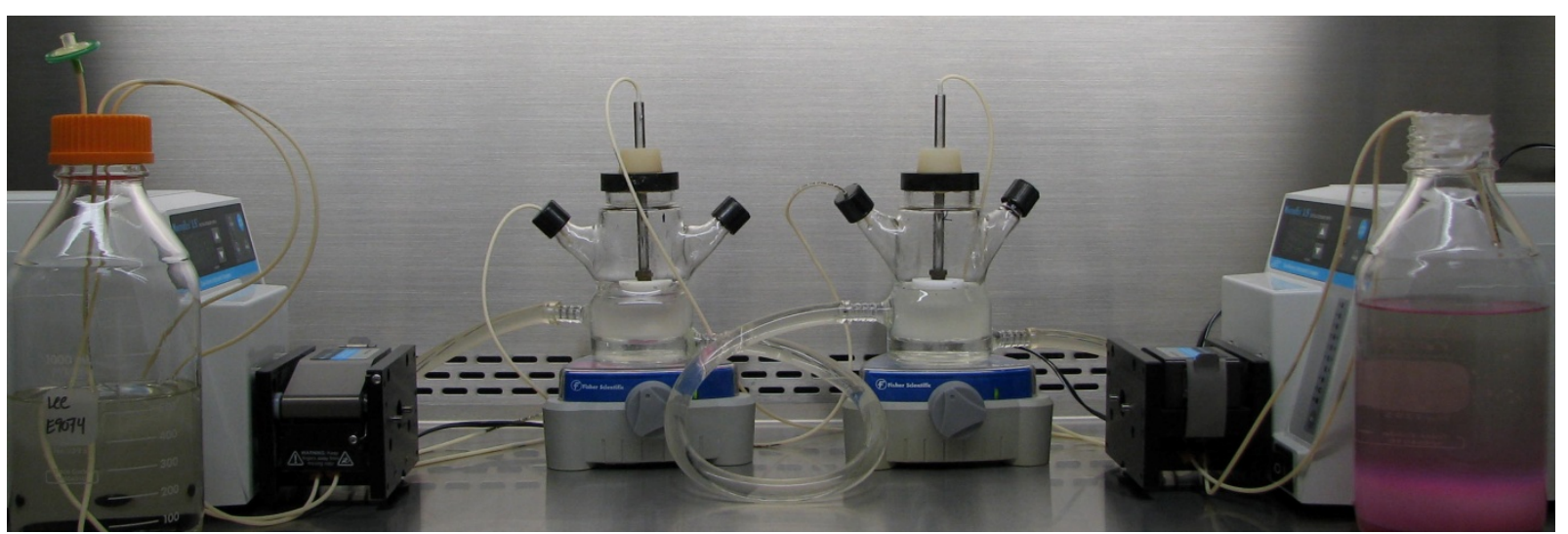

B)

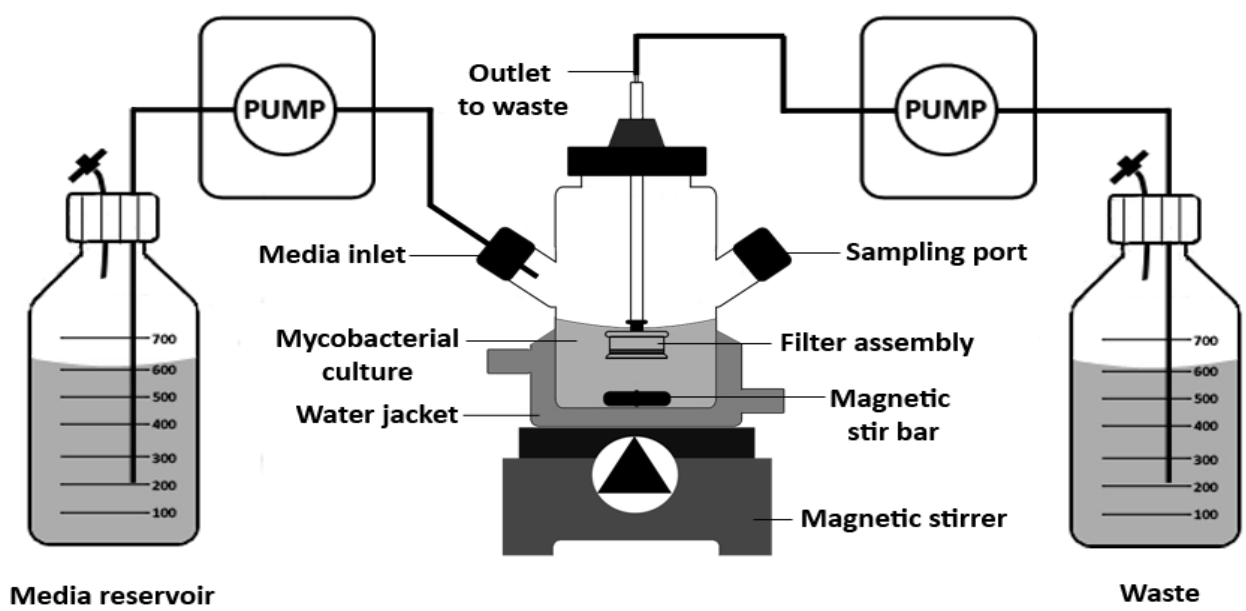

Figure 4-1. In vitro PK/PD system with two working units (Panel A) and its different components (Panel B). 


\section{Cell Viability Assay}

A rapid and sensitive microbial cell viability assay using BacTiter-Glo reagent (Promega, Madison, WI) was developed and validated to estimate the number of viable bacteria in a given sample of culture based on quantitation of the ATP present. ATP is an indicator of metabolically active cells. The homogeneous assay procedure involves adding BacTiter-Glo reagent directly to bacterial cells in medium and measuring luminescence. It utilizes a proprietary thermo stable luciferase to enable extraction of ATP from bacterial cells and to support a stable luminescent signal which can be directly measured using a luminometer. An ATP standard curve was established by making serial dilutions of ATP and recording the luminescence for each such dilution. For an unknown bacterial sample, treated with BacTiter-Glo reagent, ATP content was estimated based on the prior established ATP standard curve. A detailed protocol is described in the Promega BacTiter-Glo microbial cell viability assay technical bulletin (145).

Fresh media was inoculated with a logarithmically growing culture to obtain dilutions of $\sim 10^{3} \mathrm{CFU} / \mathrm{mL}$. This media was allowed to grow in the in vitro PK/PD system as well as in a shaker-incubator maintained at $37^{\circ} \mathrm{C}$ set to $100 \mathrm{rpm}$. Aliquots were taken on different days until the bacteria reached stationary phase. The number of viable bacteria in each sample was determined by plating serial dilutions of the samples on antibiotic-free Middlebrook $7 \mathrm{H} 11$ agar plates and counting the $\mathrm{CFU} / \mathrm{mL}$ after incubating these plates at $37{ }^{\circ} \mathrm{C}$ for 21 days. Simultaneously, ATP content present in each sample was measured using the previously described BacTiter-Glo assay. The relationship between measured ATP content and the CFU/mL was established using a mathematical equation by plotting $\log \mathrm{CFU} / \mathrm{mL}$ versus $\log \mathrm{ATP}(\mathrm{nM})$. This equation was further used to determine the $\mathrm{CFU} / \mathrm{mL}$ based on the ATP present in the unknown samples.

\section{Time-Kill Curves of Lee1445}

M. bovis $\mathrm{BCG}$, which is an attenuated derivative of Mycobacterium bovis, a virulent tubercle bacillus very closely related to M. tuberculosis (146), was used to determine the time-kill curves of Lee1445. M. bovis BCG was grown to early logarithmic growth phase in Middlebrook7H9 medium and was then used to inoculate the medium in the in vitro model. The final density of the culture in the in vitro system was $\sim 10^{6}$ $\mathrm{CFU} / \mathrm{mL}$. The medium was drawn from the flask via the combination of a $5 \mu \mathrm{m}$ and a $0.22 \mu \mathrm{m}$ filter at a predetermined flow rate that mimicked the in vivo elimination half-life of Lee1445. Fresh sterile medium was pumped into the flask at the same rate through one side arm to keep the volume of the medium at the initial level. The addition of Lee1445 doses was started when the culture had reached the logarithmic growth phase which was determined by the optical density of the culture. Lee1445 doses were added daily for 7 days to simulate in vivo plasma concentration-time profiles in rats. Different total daily doses were administered as once daily (QD), twice daily (BID), and thrice daily (TID) administration as shown in Table 4-1. Figure 4-2 shows the in vivo concentration-time profiles based on rat $\mathrm{PK}$ data that were simulated in the in vitro $\mathrm{PK} / \mathrm{PD}$ system. Although an in vivo elimination half-life of $0.43 \mathrm{hr}$ was observed for Lee1445 in rats, due to the 
Table 4-1. Dosing regimens tested in the in vitro PK/PD model.

\begin{tabular}{cccccc}
\hline $\begin{array}{c}\text { Dose } \\
\text { group }\end{array}$ & $\begin{array}{c}\text { Half-life } \\
\mathbf{h r}\end{array}$ & $\begin{array}{c}\text { Dose } \\
\mathbf{m g} / \mathbf{K g}\end{array}$ & Frequency & $\begin{array}{c}\text { Total daily dose } \\
\mathbf{m g} / \mathbf{K g} / \mathbf{d a y}\end{array}$ & $\begin{array}{c}\text { Unbound } \mathbf{C}_{\mathbf{0}} \\
\mathbf{m g} / \mathbf{L}\end{array}$ \\
\hline Control & - & - & - & - & - \\
Treatment & 1.26 & 10 & QD & 10 & 15.0 \\
& 1.26 & 50 & QD & 50 & 75.1 \\
& 1.26 & 0.2 & BID & 0.4 & 0.3 \\
& 1.26 & 1 & BID & 2 & 1.5 \\
& 1.26 & 5 & BID & 10 & 7.5 \\
& 1.26 & 25 & BID & 50 & 37.5 \\
& 1.26 & 3.33 & TID & 10 & 5.0 \\
& 1.26 & 16.67 & TID & 50 & 25.0 \\
\hline
\end{tabular}

Note: $\mathrm{C}_{0}$ : Concentration at time zero 
A)

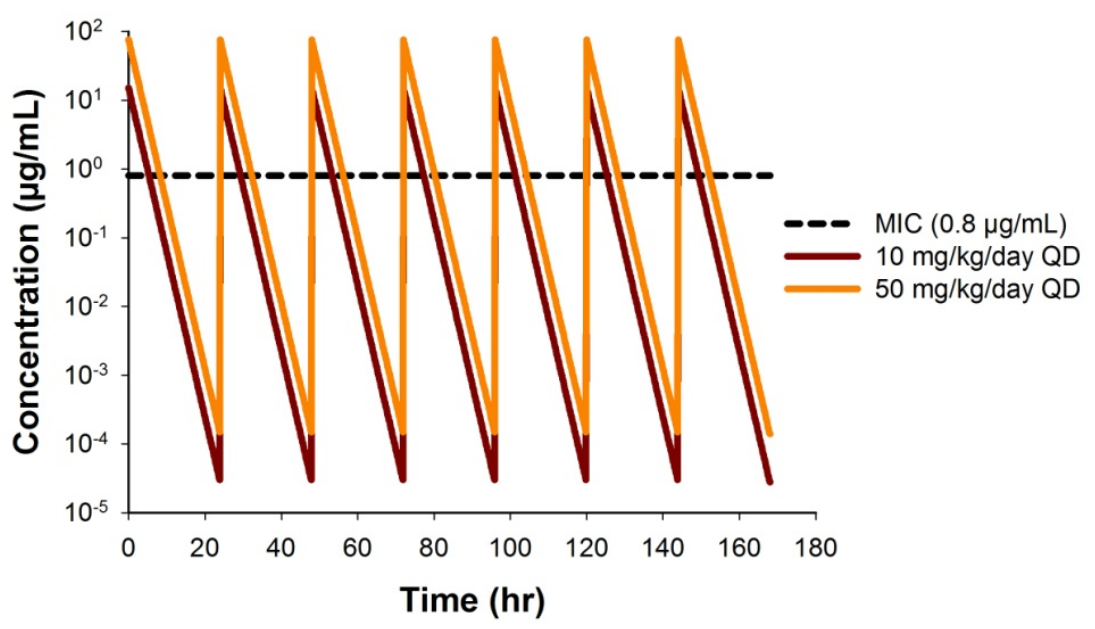

B)

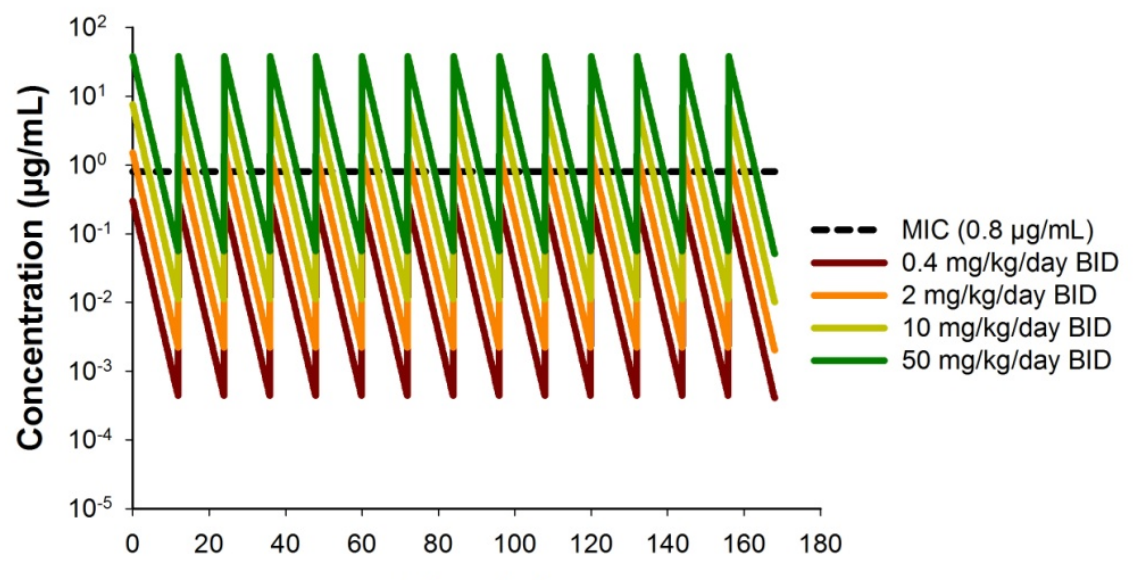

C)

Time (hr)

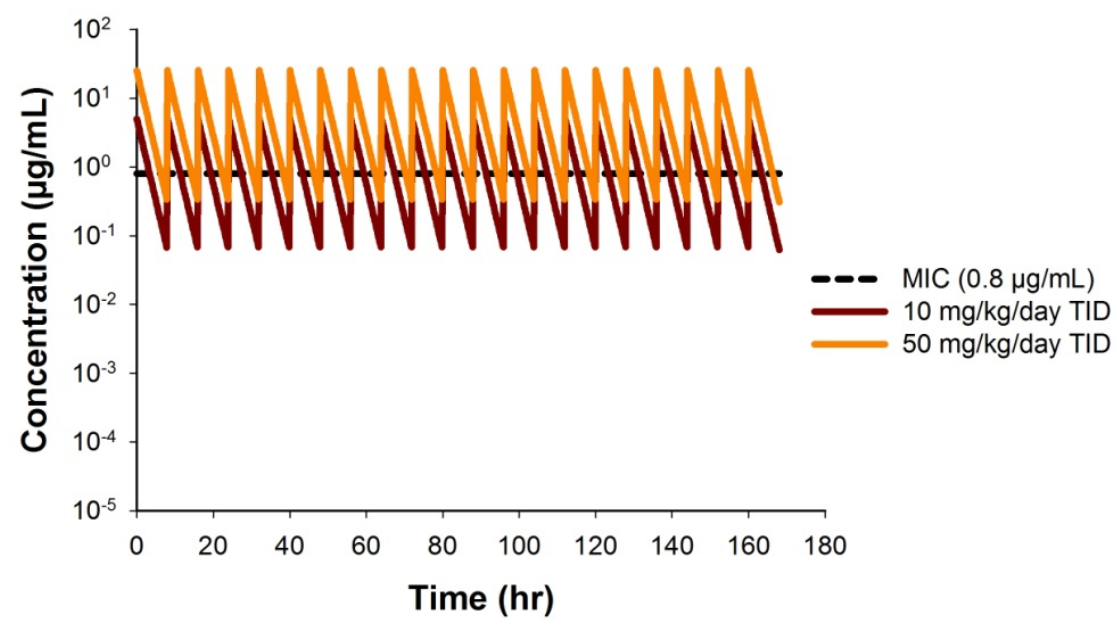

Figure 4-2. In vivo concentration-time profiles of QD regimens (Panel A), BID regimens (Panel B), and TID regimens (Panel C) simulated in the in vitro $\mathrm{PK} / \mathrm{PD}$ system. MIC of Lee 1445 against $M$. bovis BCG was $0.8 \mu \mathrm{g} / \mathrm{mL}$ represented in dotted lines. 
technical difficulties in operating the pump at higher flow rates limited by the surface area of the filter, an adjusted elimination half-life of $1.26 \mathrm{hr}$ was simulated in the in vitro model. Approximately $200 \mu \mathrm{L}$ of the sample was collected at time zero and every day for QD, every $12 \mathrm{hr}$ for BID and every $8 \mathrm{hr}$ for TID treatments thereafter until 7 days. The number of viable bacteria in each sample was determined using the ATP measurements from the sample as a surrogate for estimating CFU/mL using the previously described cell viability assay. Simultaneously, serial dilutions of the samples for certain time points were plated on antibiotic-free Middlebrook $7 \mathrm{H} 11$ agar plates to ensure that the $\mathrm{CFU} / \mathrm{mL}$ estimates obtained using ATP measurements were accurate. The antibacterial activity was measured as the reduction in number of viable bacteria, expressed as CFU/mL. Nodrug control was included in the study to assess the extent of killing by Lee1445.

\section{Time-Kill Data Analysis}

The PK/PD relationship between Lee1445 exposure and microbial kill was modeled using a previously described time-kill curve equation (129),

$$
\frac{d N}{d t}=\left[k_{0} \cdot\left(1-\frac{N}{N_{\max }}\right)-\left(1-e^{-\alpha \cdot t}\right) \cdot\left(\frac{I_{\max } \cdot C}{I C_{50}+C}\right)\right] \cdot N
$$

where $\mathrm{N}$ is the $M$. bovis $\mathrm{BCG}$ cell count in $\mathrm{CFU} / \mathrm{mL}, \mathrm{k}_{0}$ is the bacterial net growth rate constant, $\mathrm{N}_{\max }$ is the maximum number of bacteria in the system in $\mathrm{CFU} / \mathrm{mL}, \mathrm{I}_{\max }$ is the maximum kill rate, $\mathrm{C}$ is the concentration of Lee1445 at time $\mathrm{t}$, and $\mathrm{IC}_{50}$ is the concentration at half-maximal kill rate. The logistic growth function, 1-N/N $\mathrm{N}_{\max }$ was used to describe the limited growth of bacteria in the absence of antibacterial agents. The delay rate constant $\alpha$ was incorporated to characterize the initial delay in the killing. All timekill data for different dosing regimens were analyzed simultaneously by nonlinear mixed effects modeling (NONMEM v.6.2, Icon, Ellicott City, MD). PK parameters were fixed in the PK/PD model and set equal to their selected values $(\mathrm{V}=55 \mathrm{~mL}$, where $\mathrm{V}$ is volume of the media in the system and half-life, $\mathrm{t}_{1 / 2}=1.26 \mathrm{hr}$ ). First order conditional estimation with interaction in NONMEM which allows interaction between the betweenexperimental variability and residual variability was used to analyze the time-kill data generated from the in vitro $\mathrm{PK} / \mathrm{PD}$ system. The data and model predictions were log transformed and the random residual variability was modeled using an additive error term. The between experiment variability term was incorporated only for the net growth rate constant to account for differences in the net growth across different regimens. Model performance was analyzed using goodness-of-fit plots, including observed vs. predicted, weighted residuals vs. time and weighted residuals vs. predictions plots.

For identification of the most appropriate empirical PK/PD index associated with the microbial kill, cell counts on different days were analyzed using an inhibitory sigmoid $\mathrm{E}_{\max }$ model, as shown in the equation below:

$$
E=E_{\max }-\frac{\left(E_{\max }-E_{0}\right) \cdot(P K P D)^{\gamma}}{(P K P D)^{\gamma}+E C_{50}^{\gamma}}
$$


where, $\mathrm{E}$ is the observed $M$. bovis $\mathrm{BCG} \log \mathrm{CFU} / \mathrm{mL}, \mathrm{E}_{0}$ is the $\mathrm{BCG}$ cell count in the control experiment, $\mathrm{E}_{\max }$ is the maximal antimicrobial effect in $\log \mathrm{CFU} / \mathrm{mL}, \mathrm{EC}_{50}$ is the value of PK/PD index that produces half-maximal antimicrobial effect and $\gamma$ is the hill coefficient. PKPD is one of the empirical PK/PD indices frequently used in antibiotic therapy namely $\mathrm{AUC}_{0-24} / \mathrm{MIC}, \mathrm{C}_{\max } / \mathrm{MIC}$ and $\mathrm{T}>\mathrm{MIC}$. The $\mathrm{PK} / \mathrm{PD}$ index that best characterized the effect of Lee1445 on M. bovis was selected based on goodness-of-fit criteria and the visual inspection.

\section{In Vivo Pharmacokinetic Bridging Study in Mice}

Mice weighing approximately $25 \mathrm{~g}$ were obtained from Harlan Bioscience (Indianapolis, IN). The animals were kept on a $12 \mathrm{hr}$ light/dark cycle with food and water available ad libitum. Groups of mice ( $\mathrm{n}=3$ per time point) received an intraperitoneal (IP) dose of Lee1445 at a dose level of $20 \mathrm{mg} / \mathrm{Kg}$. Blood samples $(\sim 250 \mu \mathrm{L})$ were collected at predetermined time points post-dose until 6 hours. Plasma was separated immediately by centrifugation $\left(10,000 \mathrm{rpm}\right.$ for $10 \mathrm{~min}$ at $\left.4{ }^{\circ} \mathrm{C}\right)$ and stored at $-80{ }^{\circ} \mathrm{C}$ until analysis. The study protocol was approved by the institutional animal care and use committee of the University of Tennessee Health Science Center. Plasma concentrationtime data obtained from this study was analyzed by NONMEM using ADVAN3 TRANS3 subroutine where a two compartment open model with bolus input and first order output was fit to the IP plasma concentration-time data (147).

\section{Numerical Simulations}

Numerical simulation experiments were performed based on the PK/PD model to predict the bactericidal effect of Lee 1445 on M. bovis BCG at untested dosing regimens in mice, the species used for in vivo infection model of mycobacteria. The murine PK parameters of Lee1445 were obtained from the bridging study. Using the PK/PD parameters obtained from the experimental time-kill data, and the two compartmental PK parameters obtained using the mice concentration-time profile, simulations were performed to predict the time-kill effect of Lee1445 for QD, BID and TID regimens in mice at doses up to $400 \mathrm{mg} / \mathrm{Kg} /$ day. Based on the time-kill effect, optimal doses were selected for the in vivo studies performed in M. tuberculosis infected gamma knockout (GKO) mice at the Department of Microbiology, Immunology and Pathology, Colorado State University.

\section{Results and Discussion}

\section{Cell Viability Assay}

The relationship between $\log \mathrm{CFU} / \mathrm{mL}$ and $\log$ ATP was found to be linear with a regression coefficient $\left(\mathrm{R}^{2}\right)$ of 0.9853 as shown in Figure 4-3. By measuring the amount of 


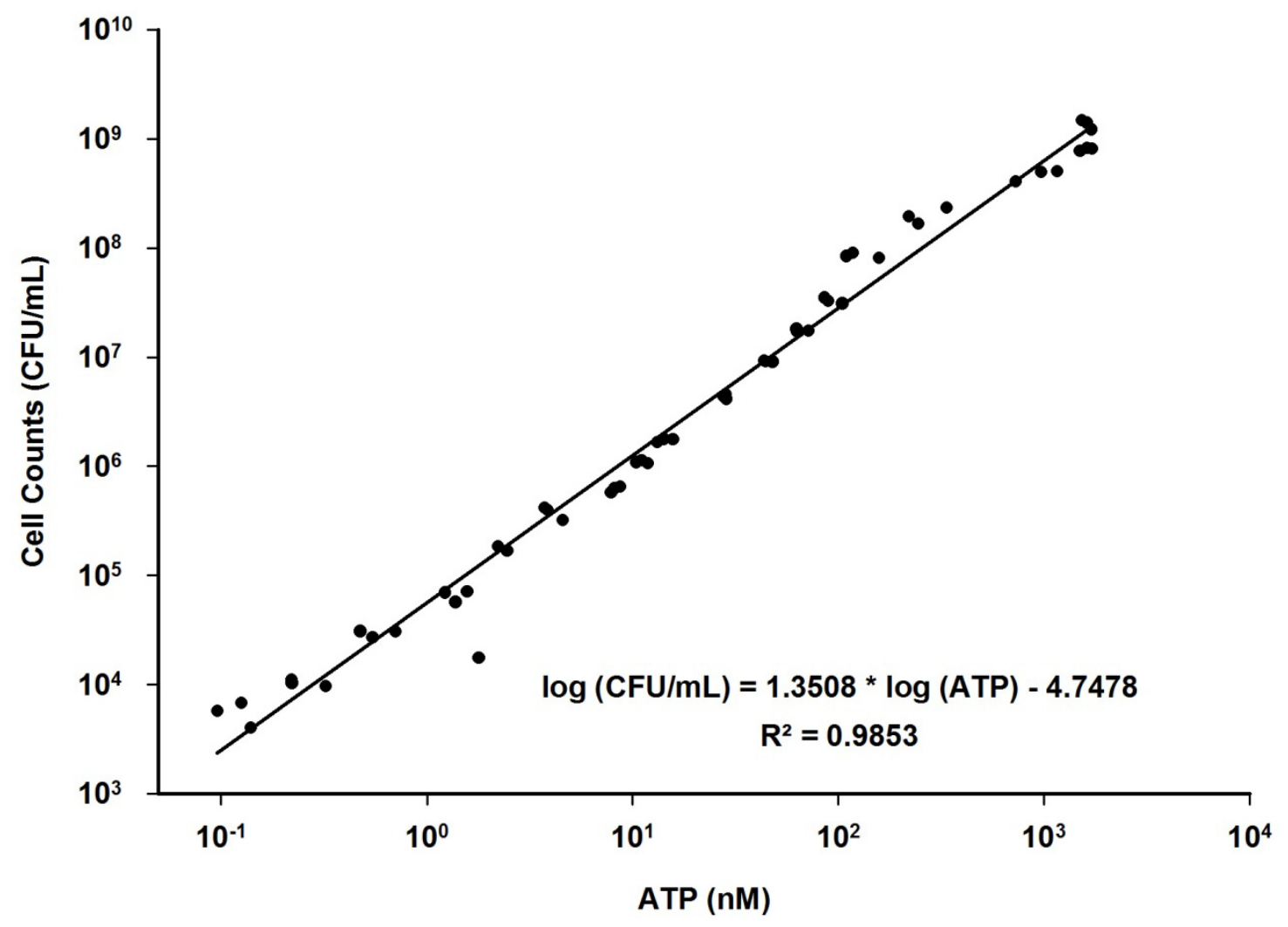

Figure 4-3. Relationship between cell counts and ATP present in a given sample. 
ATP present in a test sample, it is possible to estimate the cell viability in terms of $\mathrm{CFU} / \mathrm{mL}$. In the presence of an antibiotic that is known for its bactericidal action, the numbers of bacterial cells decrease over a period of time. This decrease in cell viability can be measured by quantifying the amount of ATP present in a sample at any given time and extrapolating that using the mathematical relationship to estimate the cell counts in $\mathrm{CFU} / \mathrm{mL}$. For a slow growing organism such as $M$. bovis $\mathrm{BCG}$, the time taken for the colonies to grow when using the commonly used plating technique is 21 days and it is important to minimize such significant amounts of wait time especially during the drug development phase to enable faster screening of novel agents. Since this assay helps in estimating the cell counts almost instantenously, it gives a significant advantage over the already existing techniques for measuring cell counts. However, caution must be taken in validating it for a newer class of agents because certain agents tend to significantly increase the luminescence and thereby the ATP counts leading to erroneous results. For example, in the presence of INH, it was found that although the M. bovis BCG cell counts decreased when treated with INH using the plating technique, ATP measurements were significantly higher leading to very high cell counts as per the mathematical relationship. Hence this assay cannot be used to measure cell viability when cells are treated with INH. However, this phenomenon was not observed with spectinamides indicating that this assay can be safely used for this class of agents.

\section{Time-Kill Curves and PK/PD Parameters}

In vitro $\mathrm{PK} / \mathrm{PD}$ models have many favorable characteristics, such as flexibility, adaptability, relatively low cost, good correlation with human and animal data and no ethical concerns as compared with animal experiments, which make them excellent experimental platforms (81). Especially with anti-TB drugs, the drug-mycobacterial interaction can be measured more precisely than in vivo since the influence of the immune system is absent in such models (137). The time-kill effect of Lee1445 against M. bovis BCG at different dosing regimens is shown in Figure 4-4. Doses $0.4 \mathrm{mg} / \mathrm{Kg} /$ day and $2 \mathrm{mg} / \mathrm{Kg}$ /day administered as BID regimens did not show any bactericidal effect and were comparable to untreated control. At much higher doses $10 \mathrm{mg} / \mathrm{Kg}$ and 50 $\mathrm{mg} / \mathrm{Kg} /$ day administered QD, Lee1445 displayed a static effect where the bacterial cells remained in a state of equilibrium with the growth rate balancing out the death rate in the flask. The same total daily dose of $10 \mathrm{mg} / \mathrm{Kg} /$ day and $50 \mathrm{mg} / \mathrm{Kg} /$ day administered as BID regimens showed a significant difference in the killing pattern compared to the same total daily dose administered as QD regimen. Also comparing the simulated PK concentrationtime profiles shown in Figure 4-2 to the time-kill curves suggests clearly that the time for which the concentrations remain above the MIC value $(0.8 \mu \mathrm{g} / \mathrm{mL})$ is nearly doubled when administered as BID compared to QD. This result clearly indicates that for better efficacy, the concentrations need to remain above MIC for longer periods of time rather than the concentrations remaining above a certain threshold value $\left(\mathrm{C}_{\max }\right)$ or the exposure associated with it (AUC). The time-kill effect of TID regimens did not differ from the BID regimens suggesting that a maximum effect was achieved although the concentrations remain above MIC for longer periods in TID regimens compared to BID regimens. From the dose ranging studies where different doses were administered at a 


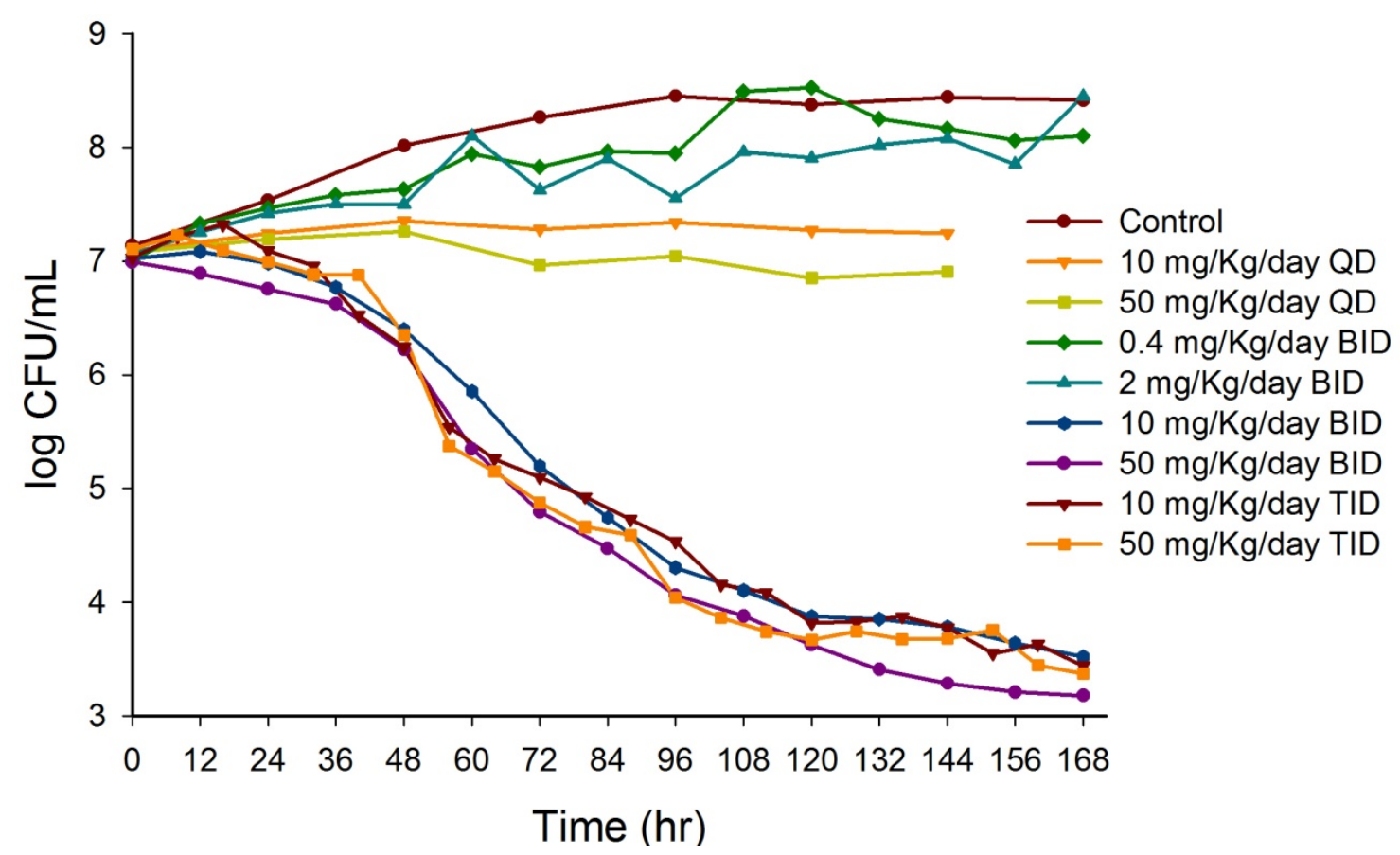

Figure 4-4. Time-kill effect of Lee1445 against $M$. bovis BCG with observed log $\mathrm{CFU} / \mathrm{mL}$ plotted against time for different dosing regimens. 
fixed interval, it is evident that higher doses produced better time-kill effect. For example in BID regimens, doses from $0.4 \mathrm{mg} / \mathrm{Kg} /$ day and up to $50 \mathrm{mg} / \mathrm{Kg} /$ day showed a significant difference in the bactericidal effect suggesting that there is clearly a dosedependent effect.

A semi-mechanistic PK/PD model incorporating a logistic function for growth of mycobacteria in the absence of drug and an inhibitory sigmoidal $\mathrm{E}_{\max }$ model with a delay function for the time-kill effect of the drug was fit to the data obtained from in vitro $\mathrm{PK} / \mathrm{PD}$ studies. The maximum growth rate constant $\left(\mathrm{K}_{0}\right)$ was estimated to be $0.0274 \mathrm{hr}^{-1}$ which corresponds to a maximum doubling time of $25.3 \mathrm{hr}$. The commonly observed in vitro doubling times when working with $M$. bovis BCG are in the range of $20-24 \mathrm{hr}$ (148) suggesting that the observed doubling time is comparable to the published range. A maximum bacterial kill rate $\left(I_{\max }\right)$ was calculated as $0.0566 \mathrm{hr}^{-1}$, suggesting that in the absence of growth, it takes nearly 12.2 hours for the bacterial counts to be halved. The concentration of the drug required to produce half-maximum inhibition $\left(\mathrm{IC}_{50}\right)$ was calculated as $2.62 \mathrm{mg} / \mathrm{L}$. Since there was a delay in the commencement of the actual killing effect, a delay function was employed in the equation. The delay rate constant for the initial kill was found to be $0.0245 \mathrm{hr}^{-1}$, suggesting the half-life of the delay in the initial kill equivalent to $28.3 \mathrm{hr}$. The results of this analysis are shown in Table 4-2 and the model predicted profiles for various dosage regimens are shown in Figure 4-5.

Examination of the relationship between empirical PK/PD indices and microbial kill for day 6 of the treatment revealed that the PK/PD index T $>$ MIC was able to describe the Lee1445 mediated microbial kill (Figure 4-6). Similar results were observed for days 3,4 , and 5 data (results not shown). $\mathrm{AUC}_{0-2} / \mathrm{MIC}$ and $\mathrm{C}_{\max } / \mathrm{MIC}$ did not show any relevance to the microbial kill on any given day indicating that it is a time dependent rather than a concentration dependent antibacterial kill. The parameter estimates from the empirical PK/PD analysis are listed in Table 4-3. This analysis suggests that the longer the free drug concentrations remain above MIC the better the microbial kill. Clinically, this can be achieved by administering the drug more frequently or by administration of higher doses so that the free drug concentrations remain above MIC for longer periods in spite of the short half-life of the drug as in the case of Lee1445.

\section{In Vivo Pharmacokinetic Bridging Study in Mice}

The efficacy studies in a tuberculosis infection model were carried out in gamma knockout (GKO) mice. Since the pharmacokinetic profile varies across species, it is important to understand the differences in the pharmacokinetic parameters between rats and mice in order to design a rational dosing regimen. To characterize the PK properties in mice, a bridging study was performed where Lee 1445 was administered intraperitoneally to healthy mice at a dose of $20 \mathrm{mg} / \mathrm{Kg}$. Figure $4-7$ shows the pharmacokinetic profile of Lee1445 in mice. A two compartmental model was fitted to the data and the pharmacokinetic parameters obtained are shown in Table 4-4. The clearance was higher in mice and the $t_{1 / 2 \alpha}$ was relatively shorter in mice compared to rats 
Table 4-2. In vitro PK/PD parameter estimates of Lee1445.

\begin{tabular}{cccc}
\hline Parameter & Units & Estimate (RSE) & $\begin{array}{c}\text { BEV } \\
(\mathbf{\% C V})\end{array}$ \\
\hline $\mathrm{K}_{0}$ & $\mathrm{hr}^{-1}$ & $0.0274(24.5)$ & 48.4 \\
$\mathrm{IC}_{50}$ & $\mathrm{mg} / \mathrm{L}$ & $2.62(90.8)$ & \\
$\mathrm{I}_{\max }$ & $\mathrm{hr}^{-1}$ & $0.0566(30.9)$ & \\
$\alpha$ & $\mathrm{hr}^{-1}$ & $0.0245(14.3)$ & \\
$\mathrm{RV}$ & $\% \mathrm{CV}$ & $22(13.8)$ & \\
\hline
\end{tabular}

Notes: RSE: Relative standard error

BEV: Between experiment variability

$\mathrm{K}_{0}$ : maximum growth rate constant in the absence of drug

$\mathrm{IC}_{50}$ : concentration needed to produce half-maximal effect

$\mathrm{I}_{\max }:$ maximum bacterial kill rate

$\alpha$ : delay rate constant

RV: Residual variability

$\mathrm{CV}$ : Coefficient of variation 


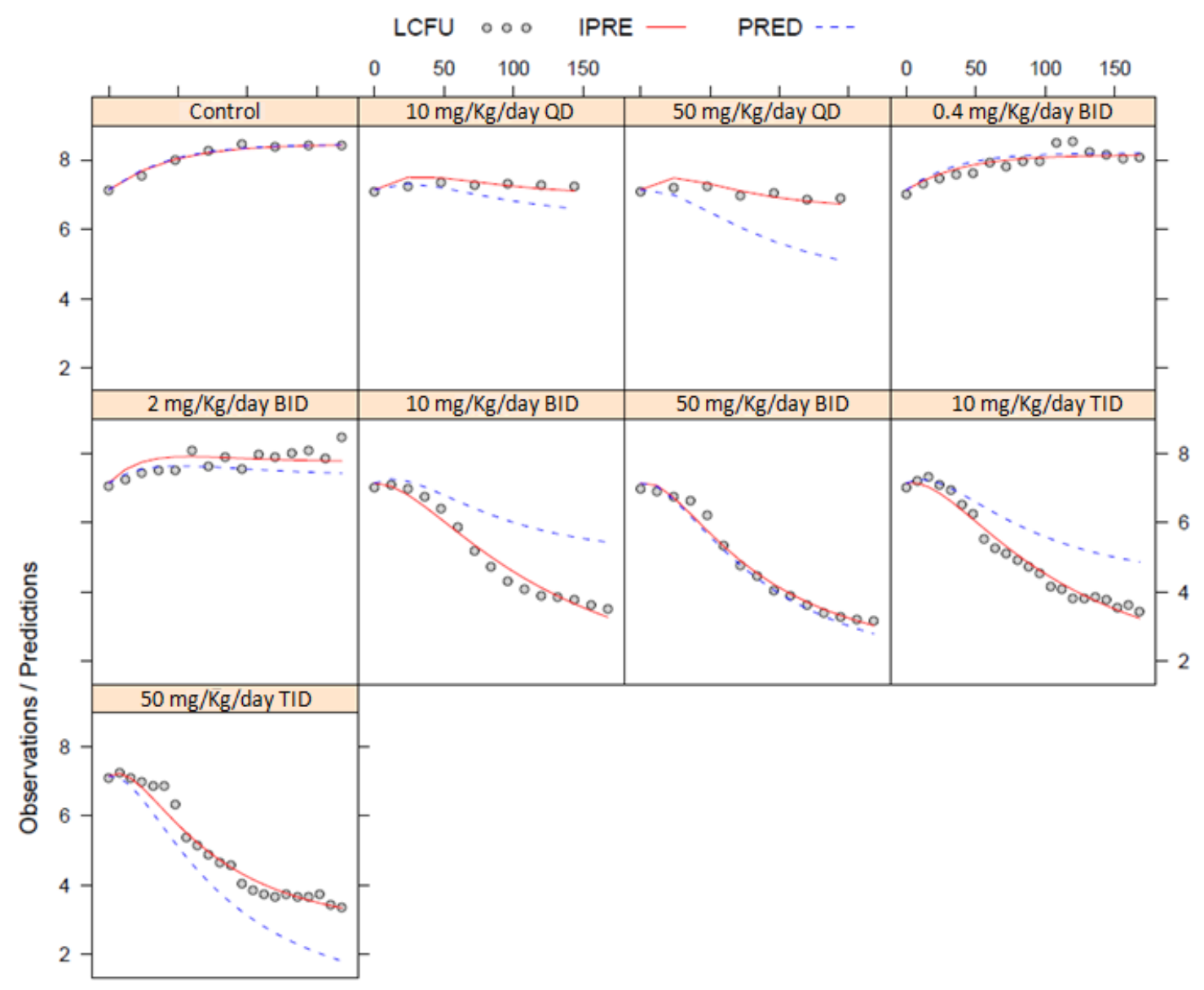

Figure 4-5. Model predicted profiles for various dosage regimens. LCFU is observed $\log \mathrm{CFU} / \mathrm{mL}$ data from in vitro $\mathrm{PK} / \mathrm{PD}$ model, IPRE is individual predicted $\log \mathrm{CFU} / \mathrm{mL}$ for each dosing regimen, $\mathrm{PRE}$ is predicted $\log \mathrm{CFU} / \mathrm{mL}$ from the mean parameters of the mathematical model. 
A)

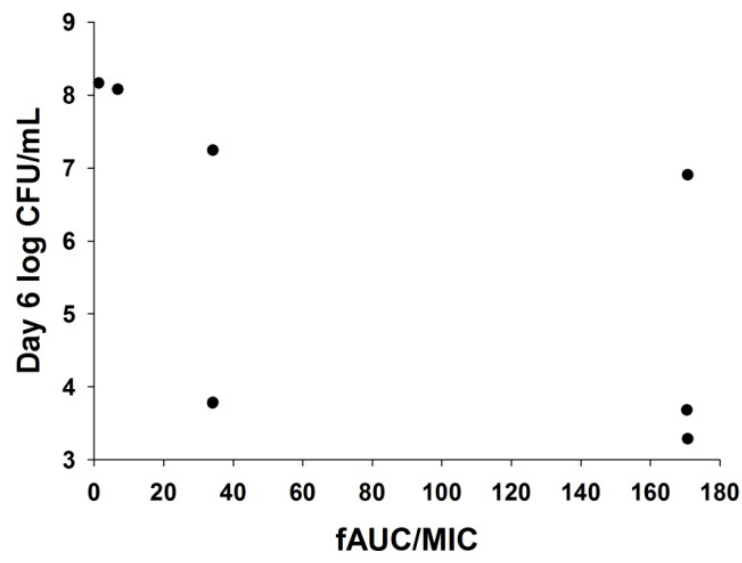

B)

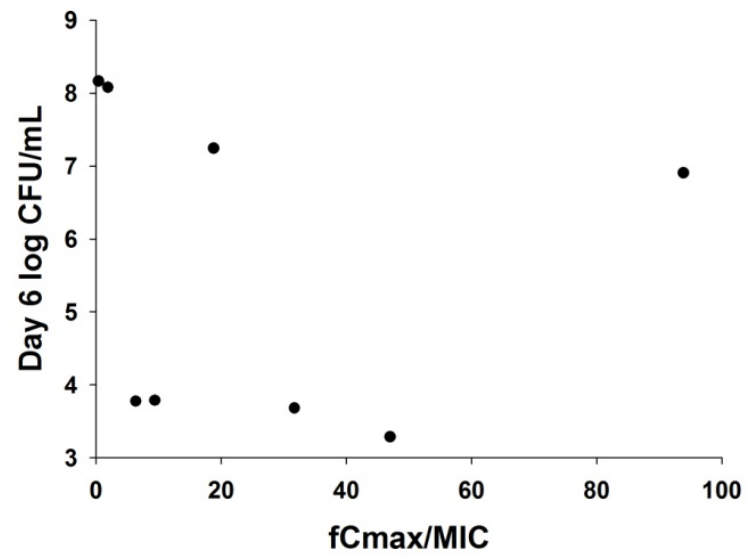

C)

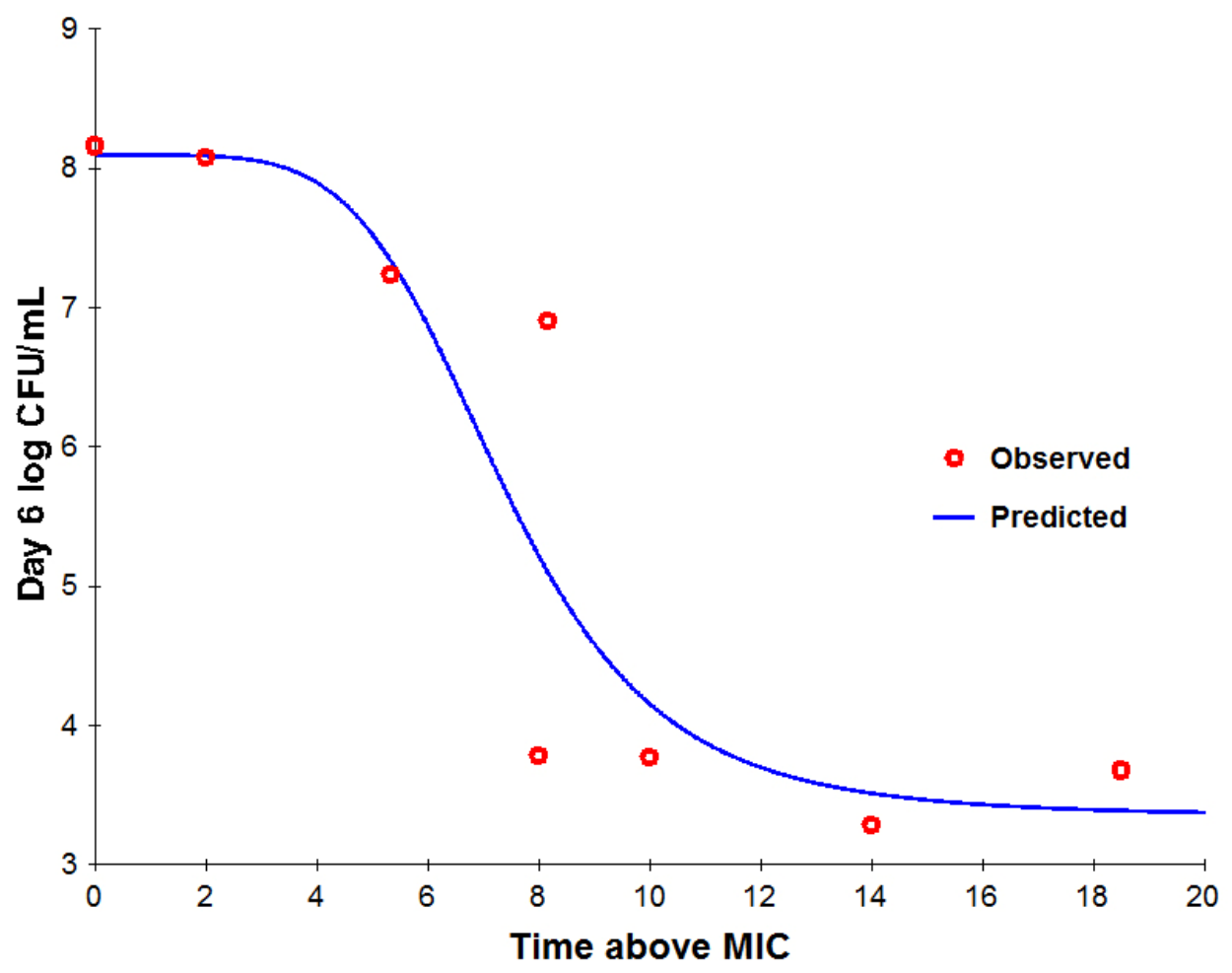

Figure 4-6. $\quad \mathrm{PK} / \mathrm{PD}$ indices and their relationship to the microbial kill on day 6 . No correlation is seen between microbial kill versus unbound $\mathrm{AUC}_{0-24} / \mathrm{MIC}$ (Panel A) or versus unbound $\mathrm{C}_{\max } / \mathrm{MIC}$ (Panel B). An inhibitory sigmoidal relationship can be seen between day 6 microbial kill and T>MIC (Panel C). 
Table 4-3. Parameters for empirical PK/PD index $\mathrm{T}>\mathrm{MIC}$ based on day 6 data.

\begin{tabular}{cccc}
\hline Parameter & Units & Estimate & \%CV \\
\hline $\mathrm{E}_{\max }$ & $\log \mathrm{CFU} / \mathrm{mL}$ & 8.09 & 10.4 \\
$\mathrm{EC}_{50}$ & $\mathrm{hr}$ & 7.38 & 18.5 \\
$\mathrm{E}_{0}$ & $\log \mathrm{CFU} / \mathrm{mL}$ & 3.34 & 30.5 \\
$\gamma$ & & 5.18 & 93.9 \\
\hline
\end{tabular}

Notes: CV: Coefficient of variation

$\mathrm{E}_{\max }$ : Initial M. bovis $\mathrm{BCG}$ count in $\log \mathrm{CFU} / \mathrm{mL}$

$\mathrm{E}_{0}$ : M. bovis $\mathrm{BCG}$ count at the end of experiment in $\log \mathrm{CFU} / \mathrm{mL}$

$\mathrm{EC}_{50}: \mathrm{T}>\mathrm{MIC}$ value that produces half-maximal antimicrobial effect

$\gamma$ : Hill coefficient 


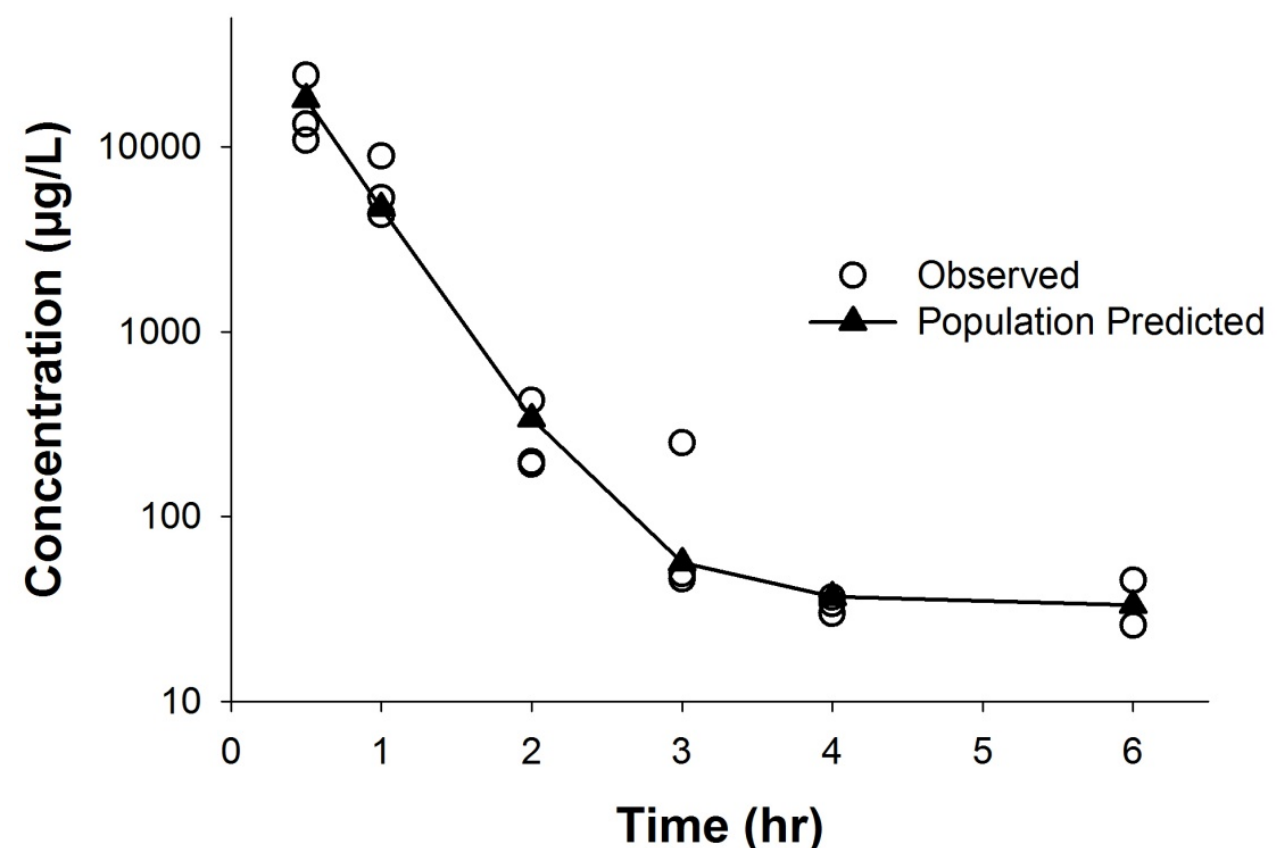

Figure 4-7. Pharmacokinetic profile of Lee 1445 in rats following $20 \mathrm{mg} / \mathrm{Kg}$ IP injection. Circles indicate observed concentrations and triangles indicate model predicted concentrations. 
Table 4-4. Lee1445 in vivo pharmacokinetic differences between rat and mouse.

\begin{tabular}{cccc}
\hline Parameter & Units & Rat & Mouse \\
\hline $\mathrm{CL}$ & $\mathrm{L} / \mathrm{hr} / \mathrm{Kg}$ & 0.56 & 0.73 \\
$\mathrm{~V}_{\mathrm{ss}}$ & $\mathrm{L} / \mathrm{Kg}$ & 1.03 & 1.26 \\
$\mathrm{t}_{1 / 2 \alpha}$ & $\mathrm{hr}$ & 0.32 & 0.25 \\
$\mathrm{t}_{1 / 2 \beta}$ & $\mathrm{hr}$ & 20.9 & 21.0 \\
\hline
\end{tabular}

Notes: CL: Total body clearance

$\mathrm{V}_{\mathrm{ss}}$ : Volume of distribution at steady state

$t_{1 / 2 \alpha}$ : Half-life associated with the macro rate constant $\alpha$

$t_{1 / 2 \beta}$ : Half-life associated with the macro rate constant $\beta$ 
suggesting that higher doses are needed in comparison to rats to achieve a given target concentration and systemic exposure.

\section{Numerical Simulations}

Utilizing the pharmacokinetic parameters from the mice PK bridging study and the pharmacodynamic parameters from the in vitro time-kill experiments, numerical simulations were performed in order to predict the bactericidal effect of Lee1445 on $M$. bovis BCG at untested dosing regimens. Since the clearance of Lee1445 was higher in mice compared to rats, higher doses were chosen for simulations in comparison to the ones tested in the in vitro PK/PD system. Figures 4-8, 4-9 and 4-10 show the predicted efficacy for QD, BID and TID dosing scenarios respectively. A dose response relationship was clearly evident in each of these dose escalation scenarios where higher doses predicted better efficacy. Figure 4-11 shows predicted efficacy profiles for a total daily dose of $200 \mathrm{mg} / \mathrm{Kg} /$ day fractionated as QD, BID and TID regimens. Even though the same total daily dose was administered, BID and TID regimens showed better efficacy compared to QD regimen. Since TID is practically cumbersome, the BID regimen was chosen over TID regimen. Hence from these simulation studies, 200 $\mathrm{mg} / \mathrm{Kg} /$ day administered BID was chosen as the optimal dose to test in the GKO mouse model. In order to evaluate the upper end of the dose response relationship in the GKO mouse model, we also recommended a higher dose of $400 \mathrm{mg} / \mathrm{Kg} /$ day BID to be incorporated in the in vivo efficacy studies.

In one of the preliminary in vivo efficacy studies of Lee1329 performed in the GKO mice infected with MTB performed at Colorado State University by our collaborators, an arbitrary dose of $200 \mathrm{mg} / \mathrm{Kg}$ QD was chosen. This dose was administered as a subcutaneous injection for nine days and CFU $/ \mathrm{mL}$ were evaluated in the lungs of the animals at the end of nine days. Results from this study are presented in Figure 4-12. Lee1329 showed approximately one log reduction in bacterial counts in the lungs compared to an untreated control. Streptomycin administered as $200 \mathrm{mg} / \mathrm{Kg}$ via the subcutaneous route served as a positive control. Lee1329 showed promising antituberculosis activity very similar to that of streptomycin in these studies. However, the dose selection criterion in these studies was arbitrary and not based on a rational $\mathrm{PK} / \mathrm{PD}$ modeling approach. There was no evidence that the dose selected would provide the optimal efficacy in these animals.

The predictions from the simulations thus suggested that total daily dose of 200 $\mathrm{mg} / \mathrm{Kg} /$ day and $400 \mathrm{mg} / \mathrm{Kg} /$ day administered BID would be optimal and result in approximately 2 and $3 \log$ reduction in bacterial counts, respectively, after seven days of therapy. Therefore these Lee1445 doses selected from the numerical simulations were evaluated in the interferon $-\gamma$ knockout mice infected with MTB. Results from this study are presented in Figure 4-13. These results indicate a dose-dependent in vivo efficacy as predicted from the numerical simulations. There was an improved reduction (from $1 \mathrm{log}$ $\mathrm{CFU} / \mathrm{mL}$ to $1.6 \log \mathrm{CFU} / \mathrm{mL}$ ) in bacterial counts in the lungs due to dose fractionation as evidenced from the in vitro $\mathrm{PK} / \mathrm{PD}$ study and the numerical simulations. Although the 


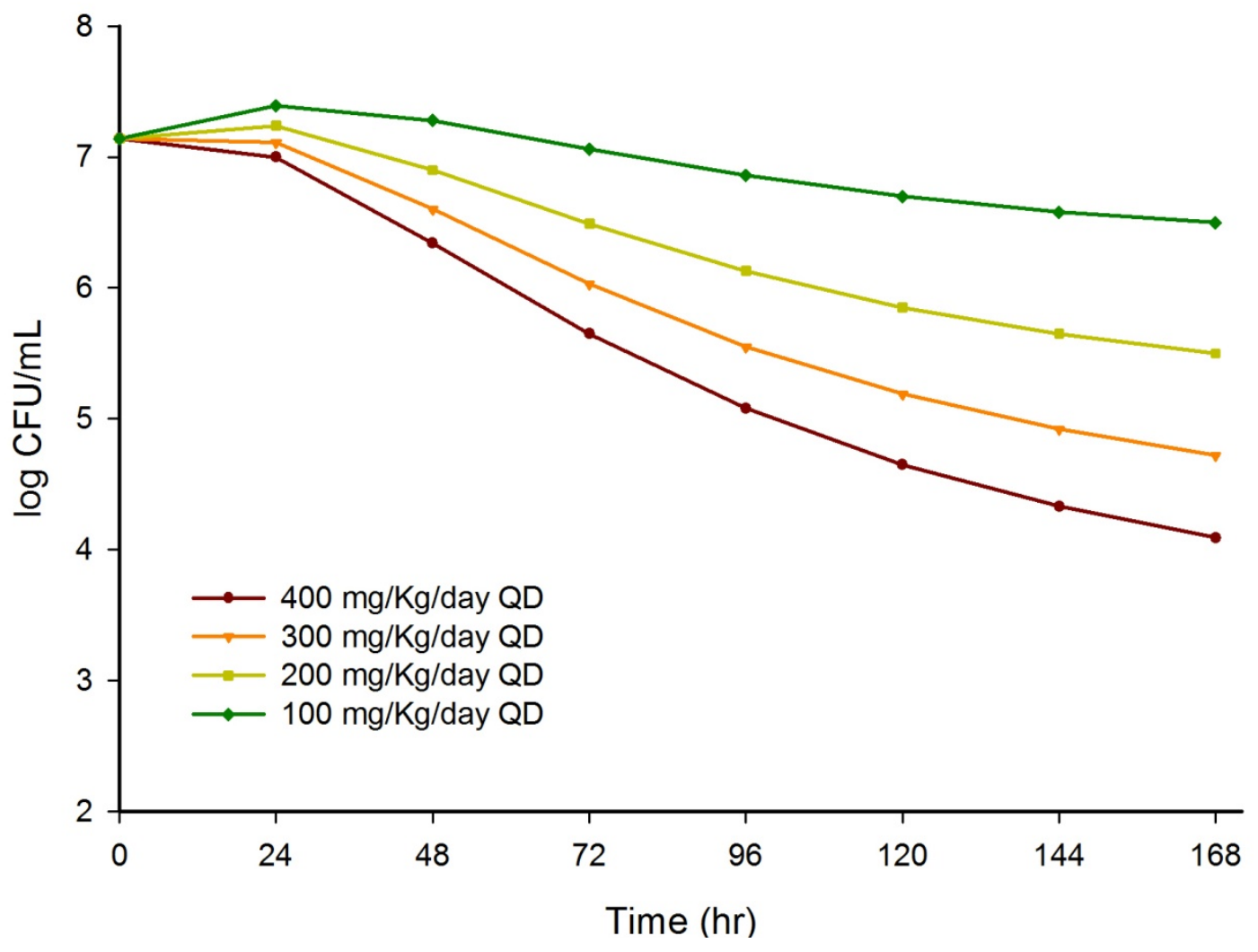

Figure 4-8. Numerical simulations for once daily dosing regimens of Lee1445 in mice. 


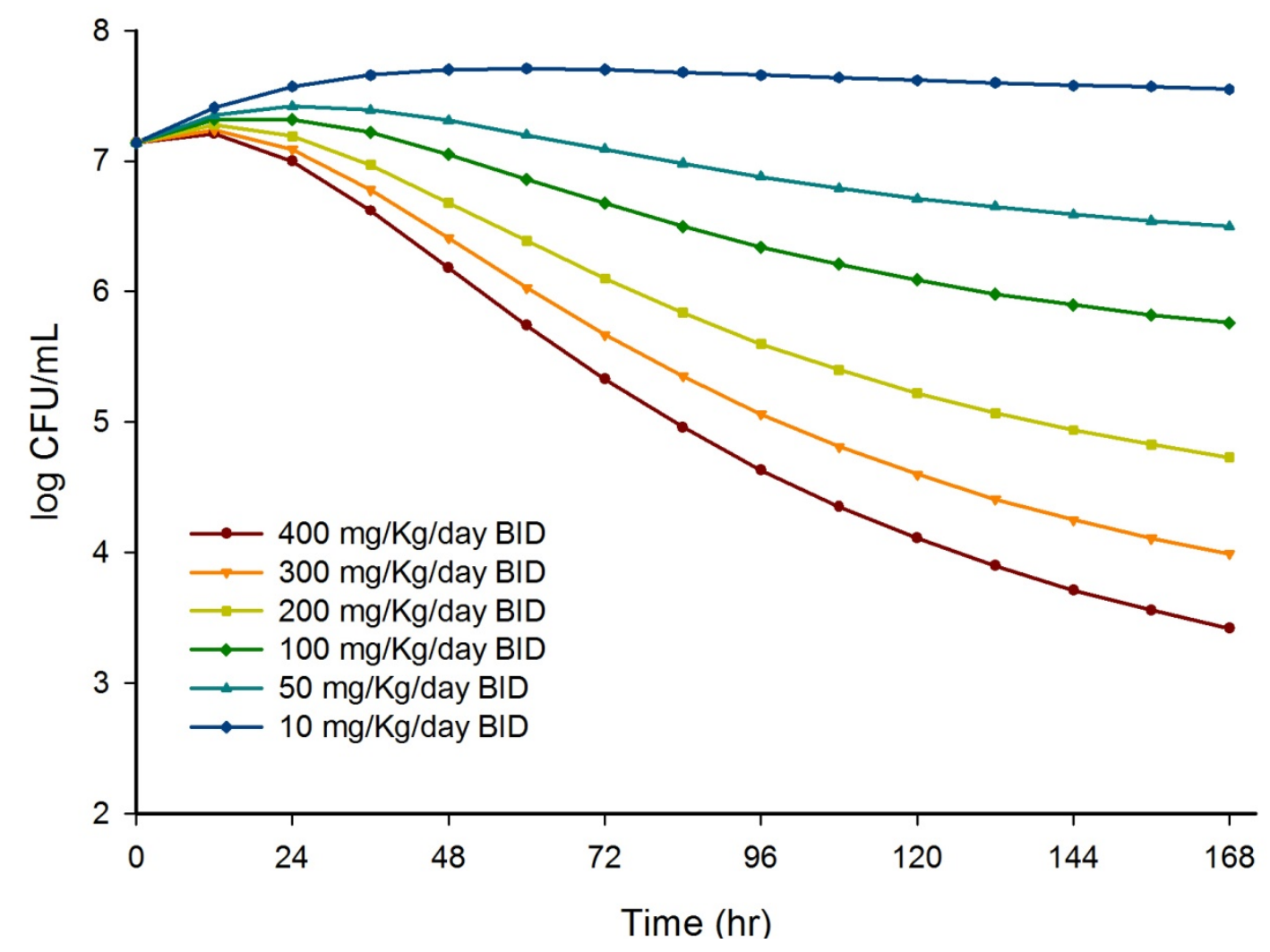

Figure 4-9. Numerical simulations for twice daily dosing regimens of Lee1445 in mice. 


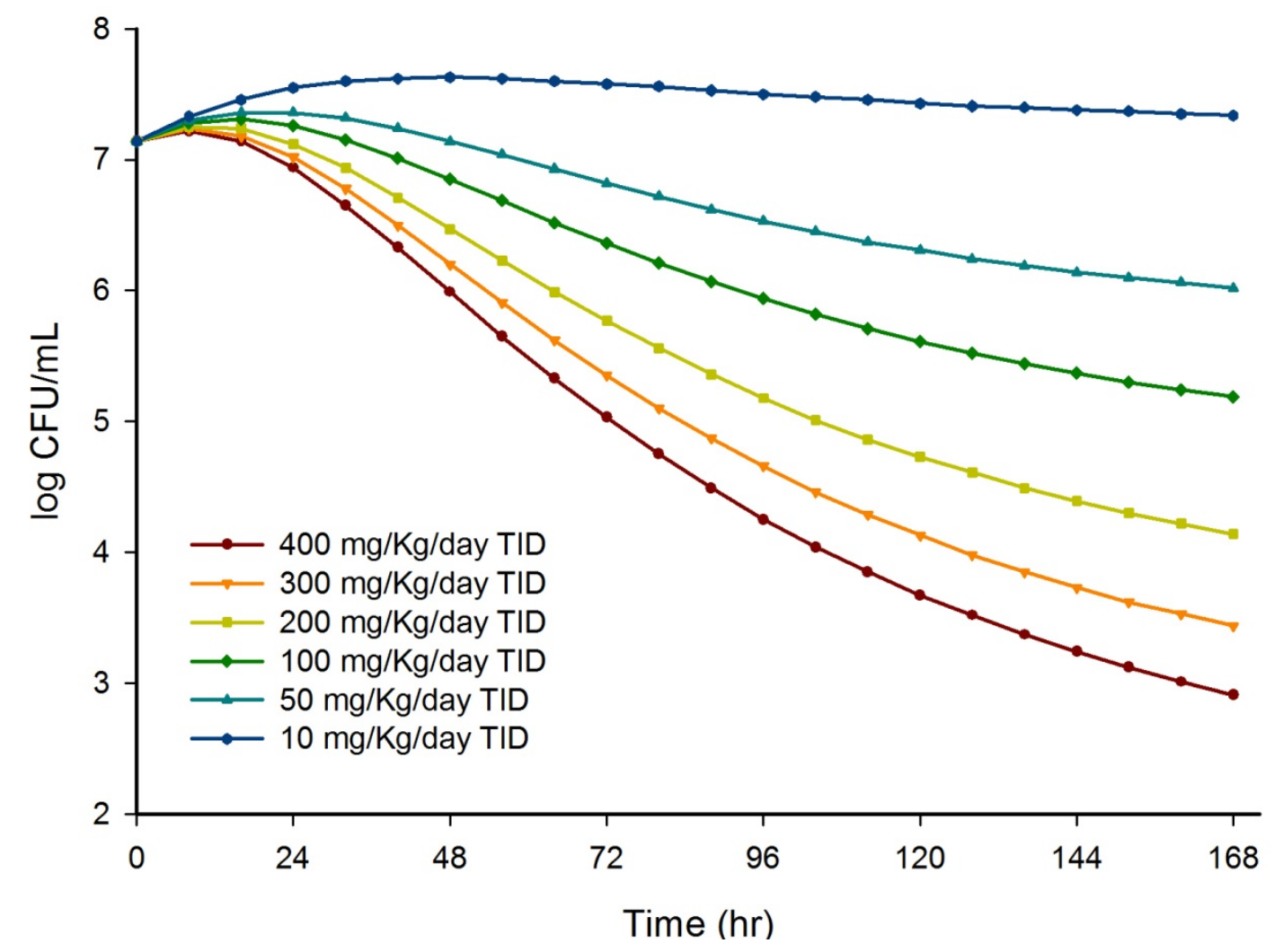

Figure 4-10. Numerical simulations for thrice daily dosing regimens of Lee1445 in mice. 


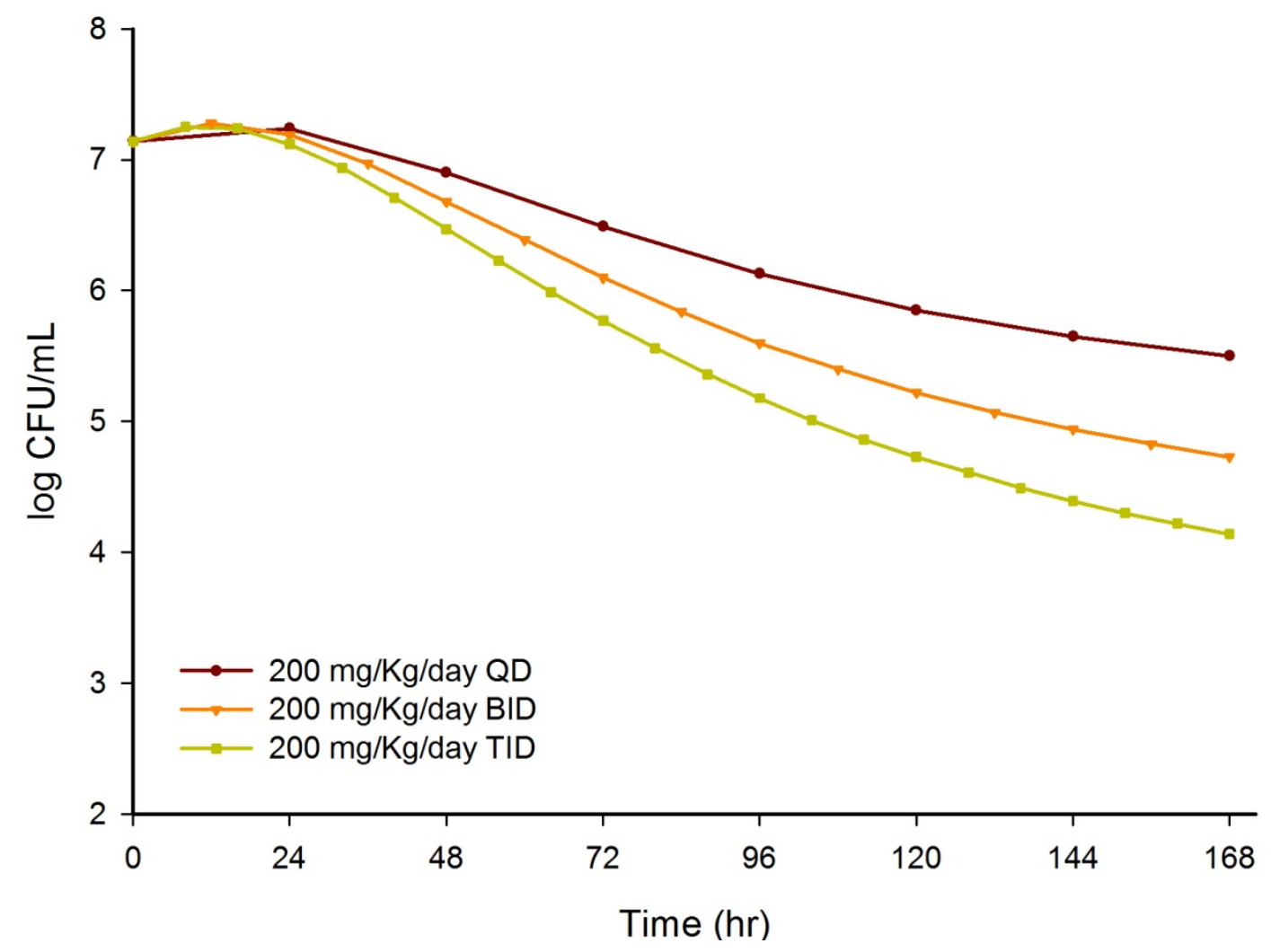

Figure 4-11. Simulated efficacy profiles of $200 \mathrm{mg} / \mathrm{Kg}$ /day administered as QD, BID and TID regimens. 


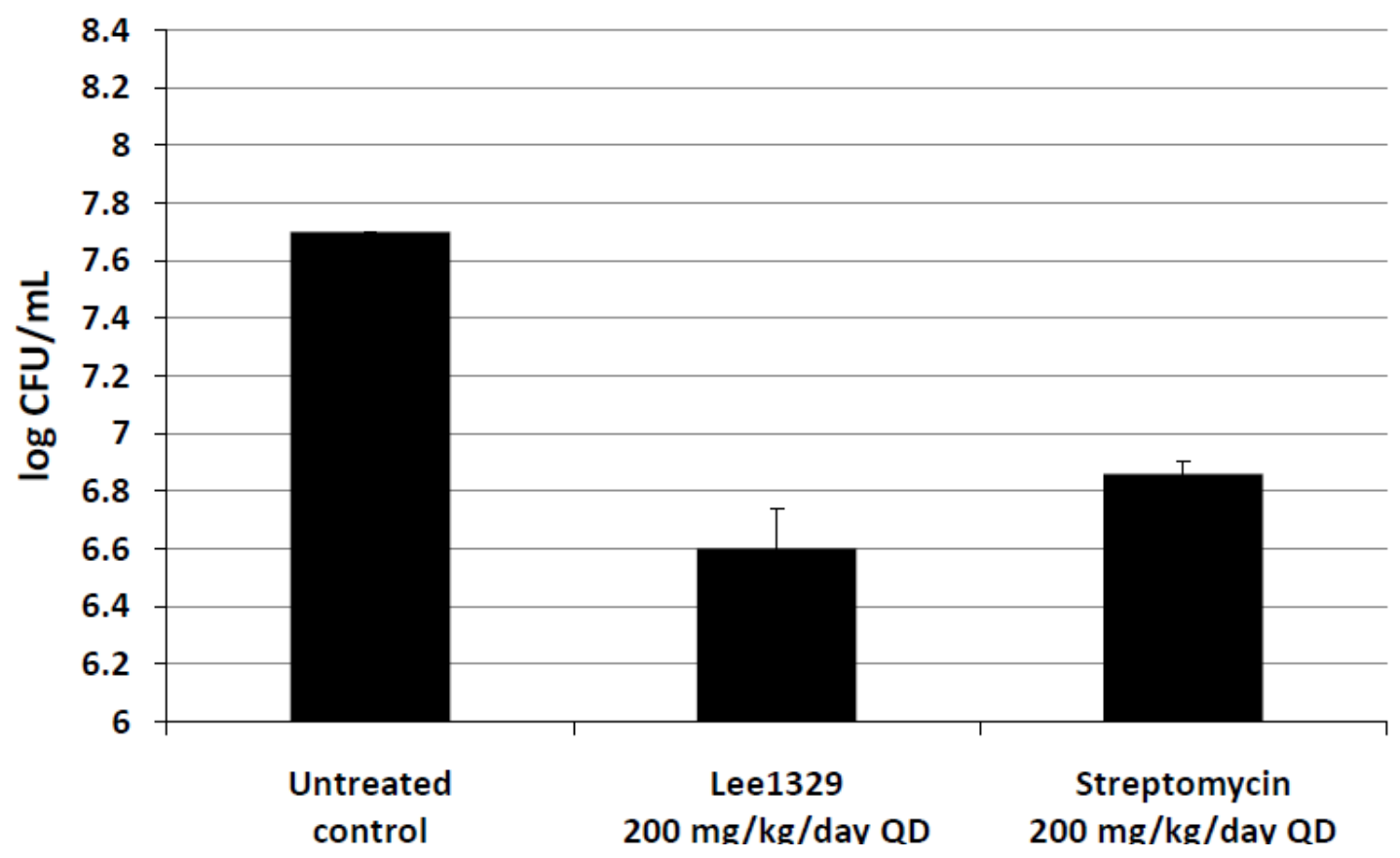

Figure 4-12. Preliminary in vivo efficacy study of Lee1329 in the GKO mouse model where the dose selection criterion was arbitrary. 


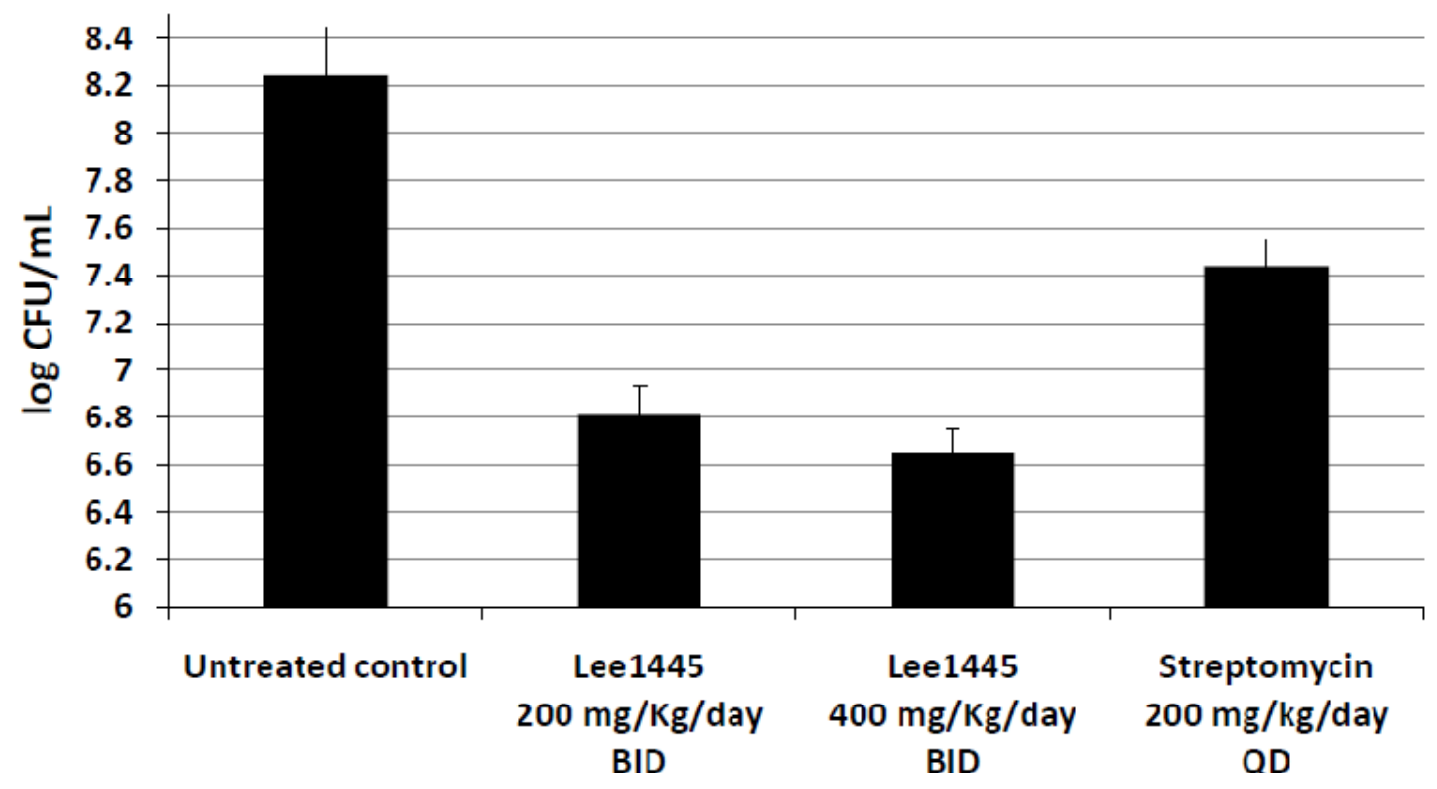

Figure 4-13. In vivo efficacy study of Lee1445 in the GKO mouse model where the dose selection criterion was based on PK/PD modeling approach. 
simulations over predicted the efficacy, they did predict a general trend that was seen in the dose-response relationship in the in vivo efficacy studies.

\section{Conclusion}

In conclusion, we developed and validated a faster way to determine the counts of colony forming units of a slow growing organism, $M$. bovis $\mathrm{BCG}$ and determined the time-kill profiles for dosing regimens of Lee1445 against this organism. A semimechanistic PK/PD model developed was able to describe the effect of Lee1445 on the growth and survival of $M$. bovis BCG. Model-based numerical simulations suggested that a twice daily dosing regimen could produce similar antibacterial effects in the GKO mice as observed in the in vitro kill curve analysis. This assumption was supported by the results obtained in the in vivo efficacy studies. These findings support the hypothesis that by using a rational $\mathrm{PK} / \mathrm{PD}$ approach and simulating the in vivo pharmacokinetic profile at different dosing regimens in an in vitro $\mathrm{PK} / \mathrm{PD}$ system facilitates characterizing the doseresponse relationship of novel anti-infectives against TB and selecting an optimal dosing regimen for maximal in vivo efficacy in animal models infected with TB. 


\section{CHAPTER 5. SUMMARY OF DISSERTATION RESEARCH}

With extensive support from several organizations, tuberculosis drug research has been attracting renewed interest after several decades of neglect. Although no new major classes of drugs have been approved for the treatment of TB since the introduction of the rifamycins into the market in 1971 (143), new and promising drug candidates have been in the discovery to late drug development phases over the past 10 years that provided hope to improve the treatment and control this dreadful threat. However, the situation is critical with increasing incidence of TB infections and growing numbers of patients with multidrug resistant TB and extensively drug resistant TB. Currently used treatment strategies involve multiple drugs with treatment periods ranging from six to nine months and especially patients with MDR-TB undergo treatment up to two years with more toxic and expensive drugs with poor patient compliance. Hence there is a definite need for more effective and better tolerated antituberculosis agents to go into optimized, less complicated and shorter dosage regimens.

In an effort to develop more potent drugs for the treatment of TB, a novel class of antituberculosis agents called spectinamides, with promising in vitro activity against MTB, has been recently characterized by our group. One of the main objectives of this work was to characterize the biopharmaceutic and pharmacokinetic properties of some of the active spectinamides and develop a rational screening paradigm which will help in the early drug development process. I hypothesized that designing a screening paradigm with ADME and drug uptake assays into mycobacterium as well as macrophages would facilitate the identification and characterization of lead compounds specific for antituberculosis therapy. To address this hypothesis, I utilized a series of existing in vivo and in vitro assays such as plasma protein binding, microsomal metabolic stability and in vivo pharmacokinetics, and developed novel drug uptake assays into M. bovis BCG and J774 murine macrophage to identify and characterize lead molecules from this novel spectinamide series of antituberculosis agents.

From the results of these assays, we found that spectinamides exhibit low to intermediate plasma protein binding and are metabolically stable with the exception of Lee1540. Following intravenous administration, spectinamides are relatively widely distributed ( 0.36 to $1.15 \mathrm{~L} / \mathrm{Kg})$ with short half-lives $(0.43$ to $0.62 \mathrm{hr})$ and the mean systemic clearance ranging between 0.36 and $0.89 \mathrm{~L} / \mathrm{hr} / \mathrm{Kg}$ with a significant fraction of drug eliminated unchanged in urine (0.46 to 1.0$)$. Spectinamides except for Lee1540 exhibit a renal excretion ratio greater than 1 indicating filtration and active secretion as the net renal elimination process. In the uptake experiments, spectinamides exhibited 3-4 times higher uptake into murine macrophages compared to streptomycin and showed nearly four times higher uptake into $M$. bovis BCG compared to spectinomycin, which may in part explain their increased activity compared to spectinomycin. Thus, we characterized the pharmacokinetic properties of key compounds in the spectinamide series and developed a screening paradigm that could be used for further optimization of leads specifically against MTB. 
Another important objective was to investigate the time course of bacterial kill of Lee1445 using an in vitro PK/PD system in order to identify the optimal dosing regimen as well as to identify the empirical PK/PD index which correlates with the therapeutic outcome. I hypothesized that simulating the in vivo pharmacokinetic profile at different dosing regimens in an in vitro $\mathrm{PK} / \mathrm{PD}$ system helps in charactering the dose-response relationship which further facilitates rational dose selection of Lee1445 for the in vivo efficacy study in animal models infected with TB. To achieve this objective, PK concentration-time profiles were simulated on the basis of the in vivo clearance of rats and the time-kill effect of different daily doses of Lee1445 were studied on the growing M. bovis $\mathrm{BCG}$ present in the PK/PD system. A novel and rapid method to quantify the bacterial counts based on the ATP measurements was developed. A mathematical model was fit to the time-kill data and in vitro PK/PD parameters were determined. Since the in vivo efficacy model of tuberculosis infection was the gamma knock-out (GKO) mouse, a PK bridging study was performed in mice. Simulations for various dosing regimens were performed using the obtained mouse PK parameters and the in vitro PK/PD parameters, which were incorporated into a combined PK/PD model to predict the efficacy in terms of reduction of bacterial counts. Based on the results of the simulations, an optimal dose was chosen for the in vivo efficacy study.

In vitro $\mathrm{PK} / \mathrm{PD}$ studies showed that $\mathrm{QD}$ was marginally effective and the same total daily dose administered BID showed a marked reduction in mycobacterial counts. The parameters obtained from the semi-mechanistic PK/PD model correlated well with the physiological and microbiological parameters. The in vitro doubling time of M. bovis BCG was found to be $25.3 \mathrm{hr}$ and the half-life of the delay in the initial kill was approximately $28 \mathrm{hr}$. The concentration of the drug required to produce half-maximum inhibition $\left(\mathrm{IC}_{50}\right)$ was calculated as $2.62 \mathrm{mg} / \mathrm{L}$. The PK/PD index $\mathrm{T}>\mathrm{MIC}$ was able to describe the Lee1445 mediated microbial kill, indicating that it is a time dependent rather than a concentration dependent antibacterial kill. This analysis indicated that the longer the free drug concentrations remain above MIC the better the microbial kill would be. Since Lee1 445 has a short half-life of $0.43 \mathrm{hr}$, this analysis indicates that by administering the drug more frequently or by administration of higher doses, the free drug concentrations would remain above MIC for longer periods and thereby lead to improved efficacy.

A total daily dose of $200 \mathrm{mg} / \mathrm{Kg} /$ day and $400 \mathrm{mg} / \mathrm{Kg} /$ day of Lee 1445 administered BID was chosen from simulations based on the parameters obtained from the mouse PK bridging study and the in vitro PK/PD model. Simulations predicted that these regimens would result in approximately 2 and 3 log reduction in bacterial counts respectively after seven days of therapy. Lee1445 doses selected from the numerical simulations were evaluated in the GKO mice infected with MTB and a dose-dependent in vivo efficacy as predicted from the numerical simulations was evident. Dose fractionation lead to an improved reduction in bacterial counts in the lungs of GKO mice from 1 to 1.6 $\log \mathrm{CFU} / \mathrm{mL}$ as predicted from the numerical simulations. Although the simulations over predicted the efficacy, they did predict a general trend that was seen in the dose-response relationship in the in vivo efficacy studies. These findings support the hypothesis that by using a rational $\mathrm{PK} / \mathrm{PD}$ approach and simulating the in vivo pharmacokinetic profile at 
different dosing regimens in an in vitro PK/PD system facilitates in characterizing the dose-response relationship and dose selection for the in vivo efficacy study in animals infected with TB.

In summary, we have successfully developed an iterative PK/PD guided process for lead optimization and characterization of spectinamides that uses a set of biopharmaceutic, pharmacokinetic and pharmacodynamic evaluations designed to identify the compounds suitable for further development. The utilization of PK/PD modeling and simulation techniques facilitated in characterizing the time course of the effect intensity of these antibiotics and resulted in an evidence-based dosage selection criterion that leads to a scientifically driven drug development process. The results of this study serve as an example for the optimization of lead compounds against tuberculosis. The general approach described in this dissertation, however, can also be applied for lead optimization of other chemical classes not only for treating tuberculosis but also for other bacterial infections. 


\section{LIST OF REFERENCES}

1. Global tuberculosis control. WHO report $2010 \mathrm{WHO} / \mathrm{HTM} / \mathrm{TB} / 2010.7$ (2010).

2. B. Meibohm and H. Derendorf. Pharmacokinetic/pharmacodynamic studies in drug product development. J Pharm Sci. 91:18-31 (2002).

3. S. Suryawanshi, L. Zhang, M. Pfister, and B. Meibohm. The current role of model-based drug development. Expert Opin Drug Discov. 5:311-321 (2010).

4. L. Zhang, M. Pfister, and B. Meibohm. Concepts and challenges in quantitative pharmacology and model-based drug development. AAPS J. 10:552-559 (2008).

5. N.R. Budha, R.E. Lee, and B. Meibohm. Biopharmaceutics, pharmacokinetics and pharmacodynamics of antituberculosis drugs. Curr Med Chem. 15:809-825 (2008).

6. A.D. Rodrigues. Use of in vitro human metabolism studies in drug development: An industrial perspective. Biochem Pharmacol. 48:2147-2156 (1994).

7. A.D. Rodrigues and S.L. Wong. Application of human liver microsomes in metabolism-based drug-drug interactions: In vitro-in vivo correlations and the Abbott Laboratories experience. Adv Pharmacol. 43:65-101 (1997).

8. T.N. Thompson. Optimization of metabolic stability as a goal of modern drug design. Med Res Rev. 21:412-449 (2001).

9. C.M. Masimirembwa, U. Bredberg, and T.B. Andersson. Metabolic stability for drug discovery and development: Pharmacokinetic and biochemical challenges. Clin Pharmacokinet. 42:515-528 (2003).

10. L.C. du Toit, V. Pillay, and M.P. Danckwerts. Tuberculosis chemotherapy: Current drug delivery approaches. Respir Res. 7:118 (2006).

11. D.G. Russell. Mycobacterium tuberculosis: Here today, and here tomorrow. Nat Rev Mol Cell Biol. 2:569-577 (2001).

12. Clinical and Laboratory Standards Institute. Approved standard M7-A7: Methods for dilution antimicrobial susceptibility tests for bacteria that grow aerobically. (2007).

13. M. Mueller, A. de la Pena, and H. Derendorf. Issues in pharmacokinetics and pharmacodynamics of anti-infective agents: Kill curves versus MIC. Antimicrob Agents Chemother. 48:369-377 (2004). 
14. W.A. Craig. Pharmacokinetic/pharmacodynamic parameters: Rationale for antibacterial dosing of mice and men. Clin Infect Dis. 26:1-10 (1998).

15. M.A. Borovinskaya, S. Shoji, J.M. Holton, K. Fredrick, and J.H. Cate. A steric block in translation caused by the antibiotic spectinomycin. ACS Chem Biol. 2:545-552 (2007).

16. D.J. Mason, A. Dietz, and R.M. Smith. Actinospectacin, a new antibiotic. I. Discovery and biological properties. Antibiot Chemother. 11:118-122 (1961).

17. B. Levy, J. Brown, and W. Fowler. Spectinomycin in gonorrhoea. Br J Clin Pract. 28:174-176 (1974).

18. Treatment of tuberculosis: Guidelines for national programmes. WHO/HTM/TB/2009.420 (2009).

19. N.R. Budha, R.B. Lee, J.G. Hurdle, R.E. Lee, and B. Meibohm. A simple in vitro $\mathrm{PK} / \mathrm{PD}$ model system to determine time-kill curves of drugs against mycobacteria. Tuberculosis. 89:378-385 (2009).

20. M.R. Holdiness. Clinical pharmacokinetics of the antituberculosis drugs. Clin Pharmacokinet. 9:511-544 (1984).

21. J.L. Johnson, D.J. Hadad, W.H. Boom, C.L. Daley, C.A. Peloquin, K.D. Eisenach, D.D. Jankus, S.M. Debanne, E.D. Charlebois, E. Maciel, M. Palaci, and R. Dietze. Early and extended early bactericidal activity of levofloxacin, gatifloxacin and moxifloxacin in pulmonary tuberculosis. Int J Tuberc Lung Dis. 10:605-612 (2006).

22. P. Jureen, K. Angeby, E. Sturegard, E. Chryssanthou, C.G. Giske, J. Werngren, M. Nordvall, A. Johansson, G. Kahlmeter, S. Hoffner, and T. Schon. Wild type minimal inhibitory concentration distributions for aminoglycoside and cyclic polypeptide antibiotics used for the treatment of Mycobacterium tuberculosis. $\mathbf{J}$ Clin Microbiol. 48:1853-1858 (2010).

23. R. Manganelli, E. Dubnau, S. Tyagi, F.R. Kramer, and I. Smith. Differential expression of 10 sigma factor genes in Mycobacterium tuberculosis. Mol Microbiol. 31:715-724 (1999).

24. B. McGee, R. Dietze, D.J. Hadad, L.P. Molino, E.L. Maciel, W.H. Boom, M. Palaci, J.L. Johnson, and C.A. Peloquin. Population pharmacokinetics of linezolid in adults with pulmonary tuberculosis. Antimicrob Agents Chemother. 53:39813984 (2009). 
25. E. Nuermberger and J. Grosset. Pharmacokinetic and pharmacodynamic issues in the treatment of mycobacterial infections. Eur J Clin Microbiol Infect Dis. 23:243-255 (2004).

26. C.A. Peloquin. Tuberculosis. In J.T. DiPiro, G.C. Yee, G.R. Matzke, B.G. Wells, and L.M. Posey (eds.), Pharmacotherapy: A pathophysiologic approach, McGraw-Hill Medical, New York. 1839-1856 (2008).

27. W.A. Petri. Chemotherapy of tuberculosis, Mycobacterium avium complex disease, and leprosy. In L.L. Brunton, J.S. Lazo, and K.L. Parker (eds.), Goodman and Gilman's the pharmacological basis of therapeutics, McGraw-Hill Medical, New York. 1203-1223 (2005).

28. W.M. McCormack and M. Finland. Drugs five years later. Spectinomycin. Ann Intern Med. 84:712-716 (1976).

29. M.C. McHenry and D.H. Fieker. Newer antibacterial and antimycotic drugs: Critical appraisal. Med Clin North Am. 62:873-897 (1978).

30. E. Novak, J.E. Gray, and R.T. Pfeifer. Animal and human tolerance of high-dose intramuscular therapy with spectinomycin. J Infect Dis. 130:50-55 (1974).

31. J. Davies, P. Anderson, and B.D. Davis. Inhibition of protein synthesis by spectinomycin. Science. 149:1096-1098 (1965).

32. W.J. Holloway. Spectinomycin. Med Clin North Am. 66:169-173 (1982).

33. Spectinomycin. The United States Pharmacopeial Convention, Inc. Available at http://vetmed.tamu.edu/common/docs/public/aavpt/spectinomycin.pdf (2008) (accessed 04 January 2011).

34. E.A. Abu-Basha, R. Gehring, and S.J. Albwa'neh. Pharmacokinetics and bioavailability of spectinomycin after i.v., i.m., s.c. and oral administration in broiler chickens. J Vet Pharmacol Ther. 30:139-144 (2007).

35. J.F. Caputo. Plasma pharmacokinetics of spectinomycin sulphate in calves after a single intramuscular, intravenous and subcutaneous injection at a dose of 10 $\mathrm{mg} / \mathrm{kg}$ body weight. The Upjohn Company technical report. 823: 9690-95-001 (1995).

36. L. Cuerpo and R.C. Livingston. Spectinomycin. In: Residues of some veterinary drugs in animals and foods. Monographs prepared by the forty-second meeting of the joint FAO/WHO expert committee on food additives. FAO Food Nutr Pap. 41:1-86 (1994). 
37. G. Ziv and F.G. Sulman. Serum and milk concentrations of spectinomycin and tylosin in cows and ewes. Am J Vet Res. 34:329-333 (1973).

38. A. Arrioja-Dechert. Compendium of veterinary products, CD ed. North American Compendiums Inc., Port Huron, MI. (2006).

39. A.L. Craigmill, S. Wetzlich, and S.A. Brown. Linco-Spectin R sterile solution in sheep. Part 1: Pharmacokinetics of spectinomycin after multiple IM administration at a dose of $15 \mathrm{mg} / \mathrm{kg}$ (5 mg lincomycin and $10 \mathrm{mg}$ spectinomycin $/ \mathrm{kg}$ ) to sheep. The Upjohn Company technical report. 803: 7926003 (1995).

40. W.J. Holloway. Spectinomycin. Med Clin North Am. 66:169-173 (1982).

41. W.M. McCormack and M. Finland. Drugs five years later. Spectinomycin. Ann Intern Med. 84:712-716 (1976).

42. J.G. Wagner, E. Novak, L.G. Leslie, and C.M. Metzler. Absorption, distribution, and elimination of spectinomycin dihydrochloride in man. Int Z Klin Pharmakol Ther Toxikol. 1:261-285 (1968).

43. P.J. Rettig, J.D. Nelson, and H. Kusmiesz. Spectinomycin therapy for gonorrhea in prepubertal children. Am J Dis Child. 134:359-363 (1980).

44. R. Kusumi, C. Metzler, and R. Fass. Pharmacokinetics of spectinomycin in volunteers with renal insufficiency. Chemotherapy. 27:95-98 (1981).

45. J.E. Gray. Local histologic changes following long-term intramuscular injections. Arch Pathol. 84:522-527 (1967).

46. W.P. Raab. Spectinomycin - experimental investigations on possible side-effects. Int J Clin Pharmacol Biopharm. 14:44-47 (1976).

47. E. Novak, C.A. Schlagel, L.A. LeZotte, and R.T. Pfeifer. The tolerance of high dose intravenous spectinomycin therapy in man. J Clin Pharmacol. 14:442-447 (1974).

48. E. Novak, J.E. Gray, and R.T. Pfeifer. Animal and human tolerance of high-dose intramuscular therapy with spectinomycin. J Infect Dis. 130:50-55 (1974).

49. A. Duancic, N.J. Fiumara, S. Alpert, Y.H. Lee, P.I. Tarr, B. Rosner, and W.M. McCormack. Comparison of spectinomycin hydrochloride and aqueous procaine penicillin $\mathrm{G}$ in the treatment of uncomplicated gonorrhea. Antimicrob Agents Chemother. 6:512-515 (1974). 
50. A. Schatz, E. Bugie, and S.A. Waksman. Streptomycin, a substance exhibiting antibiotic activity against gram-positive and gram-negative bacteria. Proc Soc Exper Biol Med. 55:66-69 (1944).

51. A. Schatz and S.A. Waksman. Effect of streptomycin and other antibiotic substances upon Mycobacterium tuberculosis and related organisms. Proc Soc Exper Biol Med. 57:244-248 (1944).

52. C.R. Spotts and R.Y. Stanier. Mechanism of streptomycin action on bacteria: A unitary hypothesis. Nature. 192:633-637 (1961).

53. M. Barza and R.T. Scheit. Antimicrobial spectrum, pharmacology and therapeutic use of antibiotics. Part 4: Aminoglycosides. Am J Hosp Pharm. 34:723-737 (1977).

54. NRA Special Review of (Dihydro) Streptomycin/ Penicillin Combination Products and (Dihydro) Streptomycin Products. Available at http://www.apvma.gov.au/products/review/docs/streptomycinpenicillin.pdf (1999) (accessed 16 January 2011).

55. W.G. Huber. Streptomycin, chloramphenicol, and other antibacterial agents. In L.M. Jones, N.H. Boots, and L.E. McDonald (eds.), Veterinary pharmacology and therapeutics, Iowa State University Press. 940-946 (1977).

56. H.D. Mercer, H.F. Righter, G. Gordon, and A.B. Carter. Serum concentration of penicillin and dihydrostreptomycin after their parenteral administration in swine. $\mathrm{J}$ Am Vet Med Assoc. 160:61-65 (1971).

57. A.A. Hadi, I.A. Wasfi, A.K. Bashir, and F.A. Gadir. Pharmacokinetics of streptomycin in camels. J Vet Pharmacol Ther. 21:494-496 (1998).

58. J.D. Baggot, D.N. Love, R.J. Rose, and J. Raus. The pharmacokinetics of some aminoglycoside antibiotics in the horse. J Vet Pharmacol Ther. 4:277-284 (1981).

59. W.V. MacFarlane, R.J.H. Morris, and B. Howard. Turn-over and distribution of water in desert camels, sheep, cattle and kangaroos. Nature. 197:270-271 (1963).

60. C. Jayachandran, M.K. Singh, S.D. Singh, and N.C. Banerjee. Pharmacokinetics of streptomycin with particular reference to its distribution in plasma, milk and uterine fluid of she-buffaloes. Vet Res Commun. 11:353-358 (1987).

61. E.A. Jackson and D.C. McLeod. Pharmacokinetics and dosing of antimicrobial agents in renal impairment, Part II. Am J Hosp Pharm. 31:137-148 (1974). 
62. G.L. Mandell and W.A. Petri. Antimicrobial agents: Drugs used in the chemotherapy of tuberculosis and leprosy. In J.G. Hardman, L.E. Limbird, P.B. Molinoff, R.W. Ruddon, and A.G. Goodman (eds.), Goodman and Gilman's the pharmacological basis of therapeutics, McGraw-Hill, New York, NY. 1161-1162 (1996).

63. C.W. Buggs, M.A. Pilling, B. Bronstein, J.W. Hirshfeld, L. Worzniak, and L.J. Key. The absorption, distribution, and excretion of streptomycin in man. J Clin Invest. 25:94-102 (1946).

64. S.M. Farber and H.R. Eagle. Streptomycin therapy of tuberculosis. Calif Med. 69:6-11 (1948).

65. G.E. Boxer, V.C. Jelinek, and A.O. Edison. Streptomycin; clearance and binding to protein. J Pharmacol Exp Ther. 97:93-104 (1949).

66. K.M. Coyne, A.L. Pozniak, M. Lamorde, and M. Boffito. Pharmacology of second-line antituberculosis drugs and potential for interactions with antiretroviral agents. AIDS. 23:437-446 (2009).

67. E.M. McGovern, A.H. Thomson, V.R. Balasubramanian, and R.S. Rodger. Streptomycin dose requirements in continuous ambulatory peritoneal dialysis. Nephrol Dial Transplant. 9:331-332 (1994).

68. J. Schacht. Biochemical basis of aminoglycoside ototoxicity. Otolaryngol Clin North Am. 26:845-856 (1993).

69. D. Bagger-Sjoback. Effect of streptomycin and gentamicin on the inner ear. Ann N Y Acad Sci. 830:120-129 (1997).

70. G.J. Kaloyanides. Aminoglycoside nephrotoxicity. In R.W. Schrier and C.W. Gottschalk (eds.), Diseases of the kidney, Little Brown and Co., Boston. 11311164 (1992).

71. C.A. Peloquin, S.E. Berning, A.T. Nitta, P.M. Simone, M. Goble, G.A. Huitt, M.D. Iseman, J.L. Cook, and D. Curran-Everett. Aminoglycoside toxicity: Daily versus thrice-weekly dosing for treatment of mycobacterial diseases. Clin Infect Dis. 38:1538-1544 (2004).

72. G.B. Appel. Aminoglycoside nephrotoxicity. Am J Med. 88:16S-20S; discussion 38S-42S (1990).

73. R.D. Moore, C.R. Smith, J.J. Lipsky, E.D. Mellits, and P.S. Lietman. Risk factors for nephrotoxicity in patients treated with aminoglycosides. Ann Intern Med. 100:352-357 (1984). 
74. J.S. Bertino, Jr., L.A. Booker, P.A. Franck, P.L. Jenkins, K.R. Franck, and A.N. Nafziger. Incidence of and significant risk factors for aminoglycoside-associated nephrotoxicity in patients dosed by using individualized pharmacokinetic monitoring. J Infect Dis. 167:173-179 (1993).

75. R.E. Brummett and K.E. Fox. Aminoglycoside-induced hearing loss in humans. Antimicrob Agents Chemother. 33:797-800 (1989).

76. F.O. Black and S.C. Pesznecker. Vestibular ototoxicity. Clinical considerations. Otolaryngol Clin North Am. 26:713-736 (1993).

77. E.J. Begg and M.L. Barclay. Aminoglycosides-50 years on. Br J Clin Pharmacol. 39:597-603 (1995).

78. A.R. Beaubien, S. Desjardins, E. Ormsby, A. Bayne, K. Carrier, M.J. Cauchy, R. Henri, M. Hodgen, J. Salley, and A. St Pierre. Incidence of amikacin ototoxicity: A sigmoid function of total drug exposure independent of plasma levels. Am J Otolaryngol. 10:234-243 (1989).

79. D.N. Hu, W.Q. Qui, B.T. Wu, L.Z. Fang, F. Zhou, Y.P. Gu, Q.H. Zhang, J.H. Yan, Y.Q. Ding, and H. Wong. Genetic aspects of antibiotic induced deafness: Mitochondrial inheritance. J Med Genet. 28:79-83 (1991).

80. W. McDermott. Toxicity of streptomycin. Am J Med. 2:491-500 (1947).

81. A. MacGowan and K. Bowker. Developments in PK/PD: Optimising efficacy and prevention of resistance. A critical review of $\mathrm{PK} / \mathrm{PD}$ in in vitro models. Int $\mathrm{J}$ Antimicrob Agents. 19:291-298 (2002).

82. T. Katsube, Y. Yano, Y. Yamano, T. Munekage, N. Kuroda, and M. Takano. Pharmacokinetic-pharmacodynamic modeling and simulation for bactericidal effect in an in vitro dynamic model. J Pharm Sci. 97:4108-4117 (2008).

83. E.R. Garrett, O.K. Wright, G.H. Miller, and K.L. Smith. Quantification and prediction of the biological activities of chloramphenicol analogs by microbial kinetics. J Med Chem. 9:203-208 (1966).

84. R.C. Li, M. Zhu, and J.J. Schentag. Achieving an optimal outcome in the treatment of infections. The role of clinical pharmacokinetics and pharmacodynamics of antimicrobials. Clin Pharmacokinet. 37:1-16 (1999).

85. A. Nolting, T. Dalla Costa, K.H. Rand, and H. Derendorf. Pharmacokineticpharmacodynamic modeling of the antibiotic effect of piperacillin in vitro. Pharm Res. 13:91-96 (1996). 
86. J.J. Schentag. Pharmacokinetic and pharmacodynamic surrogate markers: Studies with fluoroquinolones in patients. Am J Health Syst Pharm. 56:S21-24 (1999).

87. J.J. Schentag. Antimicrobial action and pharmacokinetics/pharmacodynamics: The use of AUIC to improve efficacy and avoid resistance. J Chemother. 11:426439 (1999).

88. R. Jayaram, S. Gaonkar, P. Kaur, B.L. Suresh, B.N. Mahesh, R. Jayashree, V. Nandi, S. Bharat, R.K. Shandil, E. Kantharaj, and V. Balasubramanian. Pharmacokinetics-pharmacodynamics of rifampin in an aerosol infection model of tuberculosis. Antimicrob Agents Chemother. 47:2118-2124 (2003).

89. R. Jayaram, R.K. Shandil, S. Gaonkar, P. Kaur, B.L. Suresh, B.N. Mahesh, R. Jayashree, V. Nandi, S. Bharath, E. Kantharaj, and V. Balasubramanian. Isoniazid pharmacokinetics-pharmacodynamics in an aerosol infection model of tuberculosis. Antimicrob Agents Chemother. 48:2951-2957 (2004).

90. R.K. Shandil, R. Jayaram, P. Kaur, S. Gaonkar, B.L. Suresh, B.N. Mahesh, R. Jayashree, V. Nandi, S. Bharath, and V. Balasubramanian. Moxifloxacin, ofloxacin, sparfloxacin, and ciprofloxacin against Mycobacterium tuberculosis: Evaluation of in vitro and pharmacodynamic indices that best predict in vivo efficacy. Antimicrob Agents Chemother. 51:576-582 (2007).

91. S. Grasso, G. Meinardi, I. de Carneri, and V. Tamassia. New in vitro model to study the effect of antibiotic concentration and rate of elimination on antibacterial activity. Antimicrob Agents Chemother. 13:570-576 (1978).

92. R.L. White. What in vitro models of infection can and cannot do. Pharmacotherapy. 21:292S-301S (2001).

93. S. Keil and B. Wiedemann. Mathematical corrections for bacterial loss in pharmacodynamic in vitro dilution models. Antimicrob Agents Chemother. 39:1054-1058 (1995).

94. C.A. White, R.D. Toothaker, A.L. Smith, and J.T. Slattery. Correction for bacterial loss in in vitro dilution models. Antimicrob Agents Chemother. 31:18591860 (1987).

95. E. Lowdin, I. Odenholt, S. Bengtsson, and O. Cars. Pharmacodynamic effects of sub-MICs of benzylpenicillin against Streptococcus pyogenes in a newly developed in vitro kinetic model. Antimicrob Agents Chemother. 40:2478-2482 (1996).

96. J. Blaser, B.B. Stone, and S.H. Zinner. Two compartment kinetic model with multiple artificial capillary units. J Antimicrob Chemother. 15 Suppl A:131-137 (1985). 
97. M.W. Garrison, K. Vance-Bryan, T.A. Larson, J.P. Toscano, and J.C. Rotschafer. Assessment of effects of protein binding on daptomycin and vancomycin killing of Staphylococcus aureus by using an in vitro pharmacodynamic model. Antimicrob Agents Chemother. 34:1925-1931 (1990).

98. D.S. Reeves. Advantages and disadvantages of an in vitro model with two compartments connected by a dialyser: Results of experiments with ciprofloxacin. J Antimicrob Chemother. 15 Suppl A:159-167 (1985).

99. T. Gumbo, A. Louie, M.R. Deziel, L.M. Parsons, M. Salfinger, and G.L. Drusano. Selection of a moxifloxacin dose that suppresses drug resistance in Mycobacterium tuberculosis, by use of an in vitro pharmacodynamic infection model and mathematical modeling. J Infect Dis. 190:1642-1651 (2004).

100. T. Gumbo, A. Louie, M.R. Deziel, and G.L. Drusano. Pharmacodynamic evidence that ciprofloxacin failure against tuberculosis is not due to poor microbial kill but to rapid emergence of resistance. Antimicrob Agents Chemother. 49:3178-3181 (2005).

101. T. Gumbo, A. Louie, W. Liu, P.G. Ambrose, S.M. Bhavnani, D. Brown, and G.L. Drusano. Isoniazid's bactericidal activity ceases because of the emergence of resistance, not depletion of Mycobacterium tuberculosis in the log phase of growth. J Infect Dis. 195:194-201 (2007).

102. T. Gumbo, A. Louie, W. Liu, D. Brown, P.G. Ambrose, S.M. Bhavnani, and G.L. Drusano. Isoniazid bactericidal activity and resistance emergence: Integrating pharmacodynamics and pharmacogenomics to predict efficacy in different ethnic populations. Antimicrob Agents Chemother. 51:2329-2336 (2007).

103. T. Gumbo, A. Louie, M.R. Deziel, W. Liu, L.M. Parsons, M. Salfinger, and G.L. Drusano. Concentration-dependent Mycobacterium tuberculosis killing and prevention of resistance by rifampin. Antimicrob Agents Chemother. 51:37813788 (2007).

104. T. Gumbo, C.S. Dona, C. Meek, and R. Leff. Pharmacokineticspharmacodynamics of pyrazinamide in a novel in vitro model of tuberculosis for sterilizing effect: A paradigm for faster assessment of new antituberculosis drugs. Antimicrob Agents Chemother. 53:3197-3204 (2009).

105. S. Srivastava, S. Musuka, C. Sherman, C. Meek, R. Leff, and T. Gumbo. Effluxpump-derived multiple drug resistance to ethambutol monotherapy in Mycobacterium tuberculosis and the pharmacokinetics and pharmacodynamics of ethambutol. J Infect Dis. 201:1225-1231 (2010).

106. B.S. Vogelman and W.A. Craig. Postantibiotic effects. J Antimicrob Chemother. 15:37-46 (1985). 
107. P.J. McDonald, W.A. Craig, and C.M. Kunin. Persistent effect of antibiotics on Staphylococcus aureus after exposure for limited periods of time. J Infect Dis. 135:217-223 (1977).

108. J.W. Mouton, M.N. Dudley, O. Cars, H. Derendorf, and G.L. Drusano. Standardization of pharmacokinetic/pharmacodynamic (PK/PD) terminology for anti-infective drugs. Int J Antimicrob Agents. 19:355-358 (2002).

109. C.Y. Chan, C. Au-Yeang, W.W. Yew, C.C. Leung, and A.F. Cheng. In vitro postantibiotic effects of rifapentine, isoniazid, and moxifloxacin against Mycobacterium tuberculosis. Antimicrob Agents Chemother. 48:340-343 (2004).

110. M.E. Levison. Pharmacodynamics of antimicrobial drugs. Infect Dis Clin North Am. 18:451-465, vii (2004).

111. I. Odenholt-Tornqvist, E. Lowdin, and O. Cars. Pharmacodynamic effects of subinhibitory concentrations of beta-lactam antibiotics in vitro. Antimicrob Agents Chemother. 35:1834-1839 (1991).

112. A.S. Ginsburg, J. Lee, S.C. Woolwine, J.H. Grosset, F.M. Hamzeh, and W.R. Bishai. Modeling in vivo pharmacokinetics and pharmacodynamics of moxifloxacin therapy for Mycobacterium tuberculosis infection by using a novel cartridge system. Antimicrob Agents Chemother. 49:853-856 (2005).

113. Clinical and Laboratory Standards Institute. M100-S20: Performance standards for antimicrobial susceptibility testing. Twentieth informational supplement (2010).

114. G.L. Drusano, W. Liu, C. Fregeau, R. Kulawy, and A. Louie. Differing effects of combination chemotherapy with meropenem and tobramycin on cell kill and suppression of resistance of wild-type Pseudomonas aeruginosa PAO1 and its isogenic MexAB efflux pump-overexpressed mutant. Antimicrob Agents Chemother. 53:2266-2273 (2009).

115. J.G. Hurdle, R.B. Lee, N.R. Budha, E.I. Carson, J. Qi, M.S. Scherman, S.H. Cho, M.R. McNeil, A.J. Lenaerts, S.G. Franzblau, B. Meibohm, and R.E. Lee. A microbiological assessment of novel nitrofuranylamides as anti-tuberculosis agents. J Antimicrob Chemother. 62:1037-1045 (2008).

116. R. Cha and M.J. Rybak. Linezolid and vancomycin, alone and in combination with rifampin, compared with moxifloxacin against a multidrug-resistant and a vancomycin-tolerant Streptococcus pneumoniae strain in an in vitro pharmacodynamic model. Antimicrob Agents Chemother. 47:1984-1987 (2003).

117. W.A. Craig. Proof of concept: Performance testing in models. Clin Microbiol Infect. 10 Suppl 2:12-17 (2004). 
118. S.N. Leonard, G.W. Kaatz, L.R. Rucker, and M.J. Rybak. Synergy between gemifloxacin and trimethoprim/sulfamethoxazole against community-associated methicillin-resistant Staphylococcus aureus. J Antimicrob Chemother. 62:13051310 (2008).

119. S.H. Zinner, J. Blaser, B.B. Stone, and M.C. Groner. Use of an in vitro kinetic model to study antibiotic combinations. J Antimicrob Chemother. 15 Suppl A:221-226 (1985).

120. V.H. Tam, A. Louie, M.R. Deziel, W. Liu, and G.L. Drusano. The relationship between quinolone exposures and resistance amplification is characterized by an inverted U: A new paradigm for optimizing pharmacodynamics to counterselect resistance. Antimicrob Agents Chemother. 51:744-747 (2007).

121. Y. Dong, X. Zhao, J. Domagala, and K. Drlica. Effect of fluoroquinolone concentration on selection of resistant mutants of Mycobacterium bovis BCG and Staphylococcus aureus. Antimicrob Agents Chemother. 43:1756-1758 (1999).

122. T. Homma, T. Hori, G. Sugimori, and Y. Yamano. Pharmacodynamic assessment based on mutant prevention concentrations of fluoroquinolones to prevent the emergence of resistant mutants of Streptococcus pneumoniae. Antimicrob Agents Chemother. 51:3810-3815 (2007).

123. B. Berger-Bachi, A. Strassle, and F.H. Kayser. Characterization of an isogenic set of methicillin-resistant and susceptible mutants of Staphylococcus aureus. Eur J Clin Microbiol. 5:697-701 (1986).

124. M. Wootton, R.A. Howe, R. Hillman, T.R. Walsh, P.M. Bennett, and A.P. MacGowan. A modified population analysis profile (PAP) method to detect hetero-resistance to vancomycin in Staphylococcus aureus in a UK hospital. J Antimicrob Chemother. 47:399-403 (2001).

125. D. Czock and F. Keller. Mechanism-based pharmacokinetic-pharmacodynamic modeling of antimicrobial drug effects. J Pharmacokinet Pharmacodyn. 34:727751 (2007).

126. S.G. Dahl, L. Aarons, U. Gundert-Remy, M.O. Karlsson, Y.J. Schneider, J.L. Steimer, and I.F. Troconiz. Incorporating physiological and biochemical mechanisms into pharmacokinetic-pharmacodynamic models: A conceptual framework. Basic Clin Pharmacol Toxicol. 106:2-12 (2009).

127. N.Q. Balaban, J. Merrin, R. Chait, L. Kowalik, and S. Leibler. Bacterial persistence as a phenotypic switch. Science. 305:1622-1625 (2004). 
128. E.I. Nielsen, A. Viberg, E. Lowdin, O. Cars, M.O. Karlsson, and M. Sandstrom. Semimechanistic pharmacokinetic/pharmacodynamic model for assessment of activity of antibacterial agents from time-kill curve experiments. Antimicrob Agents Chemother. 51:128-136 (2007).

129. Y. Li, M.H. Nguyen, S. Cheng, S. Schmidt, L. Zhong, H. Derendorf, and C.J. Clancy. A pharmacokinetic/pharmacodynamic mathematical model accurately describes the activity of voriconazole against Candida spp. in vitro. Int J Antimicrob Agents. 31:369-374 (2008).

130. D.G. Russell. Who puts the tubercle in tuberculosis? Nat Rev Microbiol. 5:39-47 (2007).

131. D.M. Monack, A. Mueller, and S. Falkow. Persistent bacterial infections: The interface of the pathogen and the host immune system. Nat Rev Microbiol. 2:747765 (2004).

132. S.Y. Eum, J.H. Kong, M.S. Hong, Y.J. Lee, J.H. Kim, S.H. Hwang, S.N. Cho, L.E. Via, and C.E. Barry, 3rd. Neutrophils are the predominant infected phagocytic cells in the airways of patients with active pulmonary TB. Chest. 137:122-128 (2010).

133. A. Dalhoff. Differences between bacteria grown in vitro and in vivo. J Antimicrob Chemother. 15 Suppl A:175-195 (1985).

134. D.L. Watson and J.A. Prideaux. Comparisons of Staphylococcus aureus grown in vitro or in vivo. Microbiol Immunol. 23:543-547 (1979).

135. H.D. Showalter and W.A. Denny. A roadmap for drug discovery and its translation to small molecule agents in clinical development for tuberculosis treatment. Tuberculosis. 88 Suppl 1:S3-17 (2008).

136. G.R. Davies and E.L. Nuermberger. Pharmacokinetics and pharmacodynamics in the development of anti-tuberculosis drugs. Tuberculosis. 88 Suppl 1:S65-74 (2008).

137. P.K. Vaddady, R.E. Lee, and B. Meibohm. In vitro pharmacokinetic/pharmacodynamic models in anti-infective drug development: Focus on TB. Future Med Chem. 2:1355-1369 (2010).

138. A.M. Ginsberg and M. Spigelman. Challenges in tuberculosis drug research and development. Nat Med. 13:290-294 (2007).

139. B. Davies and T. Morris. Physiological parameters in laboratory animals and humans. Pharm Res. 10:1093-1095 (1993). 
140. D.F. Veber, S.R. Johnson, H.Y. Cheng, B.R. Smith, K.W. Ward, and K.D. Kopple. Molecular properties that influence the oral bioavailability of drug candidates. J Med Chem. 45:2615-2623 (2002).

141. G.J. Kaloyanides. Drug-phospholipid interactions: Role in aminoglycoside nephrotoxicity. Ren Fail. 14:351-357 (1992).

142. N.A. Knechel. Tuberculosis: Pathophysiology, clinical features, and diagnosis. Crit Care Nurse. 29:34-43 (2009).

143. W.J. Burman and B.E. Jones. Treatment of HIV-related tuberculosis in the era of effective antiretroviral therapy. Am J Respir Crit Care Med. 164:7-12 (2001).

144. W.J. Burman. Issues in the management of HIV-related tuberculosis. Clin Chest Med. 26:283-294 (2005).

145. BacTiter-Glo ${ }^{\mathrm{TM}}$ Microbial Cell Viability Assay. Technical Bulletin \# 337. Available at http://www.promega.com/tbs/tb337/tb337.html (accessed 10 March 2011).

146. R. Frothingham, H.G. Hills, and K.H. Wilson. Extensive DNA sequence conservation throughout the Mycobacterium tuberculosis complex. J Clin Microbiol. 32:1639-1643 (1994).

147. A.J. Bailer. Testing for the equality of area under the curves when using destructive measurement techniques. J Pharmacokinet Biopharm. 16:303-309 (1988).

148. S. Mostowy, A.G. Tsolaki, P.M. Small, and M.A. Behr. The in vitro evolution of BCG vaccines. Vaccine. 21:4270-4274 (2003). 


\section{VITA}

Pavan Kumar Vaddady was born in East Godavari District, Andhra Pradesh, India to Sangeeta and Srinivasa Rao Vaddady in1982. He enrolled at the Birla Institute of Technology and Science (BITS), Pilani, Rajasthan, India in 1999 and obtained his Bachelor of Pharmacy (Honors) in 2003. He continued in the same university and graduated with Master of Pharmacy in 2005. In the fall of 2006, he enrolled in the graduate program in the Department of Pharmaceutical Sciences at the University of Tennessee Health Science Center, Memphis, Tennessee, USA to pursue his Ph.D. under the supervision of Dr. Bernd Meibohm. While at UTHSC, he received a number of accolades including the following: Eli Lilly Graduate Student Symposium Award in Pharmacokinetics, Pharmacodynamics, Drug Metabolism and Clinical Pharmacology and Translational Research during the FIP Pharmaceutical Sciences World Congress/ the American Association of Pharmaceutical Scientists Annual Meeting at New Orleans, LA in 2010; the American Society for Microbiology Student and Post Doctoral Fellow Travel Grant for attending the Interscience Conference on Antimicrobial Agents and Chemotherapy at Boston, MA in 2010; Travelship Award by the Clinical Pharmacology and Translational Research Section of the American Association of Pharmaceutical Scientists to attend the Annual Meeting at Los Angeles, CA in 2009; Outstanding Podium Presentation Award during PharmForum, the American Association of Pharmaceutical Scientists - Southern Regional Discussion Group Meeting at Oxford, MS in 2009 and was inducted into the Rho Chi Pharmacy Honor Society and the UTHSC Imhotep Society. He is also a member of the American Association of Pharmaceutical Scientists (AAPS), the American College of Clinical Pharmacology (ACCP), the American Society for Microbiology (ASM) and the American Society of Pharmacometrics (ASOP). During his graduate school he obtained a patent related to his dissertation research, published a book chapter and three peer-reviewed articles in scientific journals and also presented at a number of scientific meetings. He received his Ph.D. with a major in Pharmaceutical Sciences in 2011, and following graduation he joined the Merck Clinical PK/PD Research Group (Kenilworth, New Jersey) as a Senior Research Pharmacokineticist. 